\title{
Experiment and Simulation of Erosion Mechanism and Deformation Characteristics in Al6061-T6 beam due to Rhomboid Particle Impacts
}

\section{MingChao Du (D 13156394970@163.com )}

China University of Petroleum Huadong https://orcid.org/0000-0002-1694-4036

\section{Zengliang Li}

China University of Petroleum(East)

\section{Xiangwei Dong}

China University of Petroleum(East)

Chunyong Fan

China University of Petroleum(East)

Jiaqi Che

China University of Petroleum Huadong

\section{Yanwen Zhang}

China University of Petroleum(East)

\section{Research Article}

Keywords: Coupled FEM-SPH, Rhomboid particle impact beam, Erosion mechanism, Incident conditions, Deformation characteristics, Particle rotation

Posted Date: April 12th, 2021

DOl: https://doi.org/10.21203/rs.3.rs-184956/v1

License: (c) (i) This work is licensed under a Creative Commons Attribution 4.0 International License.

Read Full License 


\title{
Experiment and Simulation of Erosion Mechanism and Deformation Characteristics in Al6061-T6 beam due to Rhomboid Particle Impacts
}

Mingchao DU, Zengliang LI, Xiangwei DONG*, Chunyong FAN, Jiaqi CHE, Yanwen ZHANG

(Mechanic and Electronic Engineering, China University of Petroleum (East China),

Shandong 266580, China)

\begin{abstract}
The erosion mechanism and deformation characteristics of rhomboid-shaped particle impacting metal beam are studied. Physical experiments of rhomboid-shaped particle impacting cantilever beam and fixed-fixed beam are carried out respectively. The erosion behavior of particles and deformation characteristics of beam are captured by high-speed imaging system. Meanwhile, the numerical models of rhomboid-shaped particle impacting beam, based on FEM-SPH coupled method, are established. The effects of the geometrical parameters of the beam, the incident conditions of particle and the impact position on the elastic-plastic deformation of beam and rebound behavior of particles are further analyzed. The results show:

(1) The width of cantilever beam affects its maximum deflection and deformation; (2) The threshold value of breakdown velocity is controlled by the substrate size; (3) The increment of internal energy is basically independent of the impact position; (4) The deflection value at impact position of beam is maximized under the critical impact condition.
\end{abstract}

Key words: Coupled FEM-SPH; Rhomboid particle impact beam; Erosion mechanism; Incident conditions; Deformation characteristics; Particle rotation

Corresponding author: XiangWei Dong

College of mechanical and electronic engineering, China University of Petroleum (East China),66 Changjiang Rd, Huangdao District, Qingdao, China.

Zip code: 266580

E-mail: XWDongupc@163.com.

Tel/Fax: $+860532-86983311$ 


\section{Introduction}

As a common aluminum alloy material, A16061-T6 has high material strength, great corrosion resistance and excellent machining performance. It is widely used in industrial components with certain strength and corrosion resistance, such as shipbuilding, mechanical parts finishing and mold forming, etc. Scholars have conducted many research on testing and improving the properties of A16061-T6 alloy, such as machinability ${ }^{[1]}$, material formability ${ }^{[2,3]}$ and laser shock peening (LSP) to improve material fatigue behavior ${ }^{[4]}$, etc. As well, the damage and failure of A16061-T6 have also been studied, such as tensile fracture ${ }^{[5]}$, bullet penetrating target ${ }^{[6]}$ and solid particle erosion wear ${ }^{[7,8]}$. Among them, the mechanism of metal erosion wear is a much widely-investigated area. M. Papini team has performed a systematic study taking the impact of angular particles on Al6061-T6 as an example, starting from the study of single abrasive particle erosion on material surface ${ }^{[9]}$, extending to the erosion research of multiple abrasive particles ${ }^{[7,8]}$, and considering the situation that the abrasive particles are embedded in the material during the erosion process ${ }^{[10]}$, which revealed the mechanism of plastic crater forming, extrusion lip and chips separation. The phenomenon of the aggravation of material surface damage caused by secondary erosion is simulated by numerical model, and the minimum critical velocity of particles without embedding is predicted. M. Papini team focused on the study of the erosion of the angular particles on the material surface. In their experiments and numerical models, the material substrate was fully constrained and the substrate was ensured to a certain thickness so that no elastic deformation occurred during the erosion wear process. However, in the actual erosion wear conditions, the eroded material may be thin plate and not completely fixed, such as the unilateral fixation of a turbine blade. The influence of the substrate thickness and the elastic deformation should be considered in particle erosion study, where the erosion mechanism and movement behavior of particles will have certain differences compared with impact fixed substrate. Salman et al. ${ }^{[11]}$ studied the impact of $\mathrm{Al}_{2} \mathrm{O}_{3}$ spherical particles on steel plates with different thicknesses and found that with the increase of steel plate thickness, the number of 
broken $\mathrm{Al}_{2} \mathrm{O}_{3}$ spherical particles gradually increases. This is because the thin steel plate has elastic bending after being impacted and absorbs part of the kinetic energy of particles, so the proportion of energy used for particle crushing is low. When the thickness of steel plate exceeds a certain threshold, the stiffness of steel plate increases and the thickness effect disappears, and no bending deformation occurs during the impact process. In consequence, almost all of the initial kinetic energy of the particles is used for their own fracture, which leads to a significant increase in the number and extent of particles crushing. This shows that the thickness of the substrate and whether it is fully constrained has an obvious influence on the impact process of particles.

At present, the research on thin plate impacted by moving objects focuses more on high-speed impact conditions such as impact damage of flying objects on aircraft structure ${ }^{[12,13]}$, bullet penetration ${ }^{[14]}$, and hypervelocity impact collapse of space debris fragments on spacecraft shielding ${ }^{[15]}$, which results in large deformation or even penetration of the thin substrate. However, there are few researches on the erosion wear of the surface of thin plate substrates such as cantilever beams and fixed-fixed beams impacted by angular particles under medium and low speed conditions $(5 \mathrm{~m} / \mathrm{s} \sim 60 \mathrm{~m} / \mathrm{s})$. Cantilever beam and fixed-fixed beam, as easily deformable thin plate constructs, will undergo elastic-plastic deformation and exhibit reciprocating vibration behavior after being impacted by objects, which, in essence, is the internal conversion of energy with different forms. This process can dissipate the initial kinetic energy of the particle and greatly alleviate the damage effect caused by impact load. There are many applications based on the above phenomenon, such as the raindrops impacting piezoelectric vibrators for energy collection ${ }^{[16]}$, and different vibration signals generated after impacting fixed thin plate of materials with different properties can be used for material identification ${ }^{[17]}$. Considering that the metal thin plate impacted by angular particle will not only produce plastic deformation and crater forming but also accompanied by large elastic deformation and vibration, so it is necessary to conduct relevant research. However, the current literature is distinctly lacking investigation in 
to erosion mechanism and deformation characteristic of metal thin plate.

This paper considers the emerging with emphasis on the erosion mechanism and deformation characteristics of Al6061-T6 thin plate (cantilever beam and fixed-fixed beam) impacted by rhomboid-shaped particles. A pneumatic ejection system for solid particles was built and physical experiments of rhomboid-shaped particles impacting the Al6061-T6 thin plate were carried out. Subsequently, the numerical model based on FEM-SPH coupled algorithm was established, and the accuracy of coupled model was verified by comparing the erosion behavior, rebound law and deformation damage mechanism between experiments and simulation. Finally, the further analysis work was carried out by using the coupled model, which lays a foundation for the follow-up research.

\section{Experiments}

\subsection{Experimental System}

Pneumatic ejection and imaging system for solid particles is shown in Fig.1, which consists of three parts: high-pressure pneumatic launch source, solid particle launcher, and observation and recording system. Among them, the high-pressure pneumatic launch source is composed of high-pressure gas chamber, necking, normally closed solenoid valve, pipe valve connector, launch tube and base support frame, as shown in Fig. 1. The rated gas storage pressure of the high-pressure gas chamber is $30 \mathrm{MPa}$, and the impact velocity of solid particles can be adjusted by changing the gas storage pressure. The solid particle launcher consists of gun barrel with variable cross-section, particle loader, substrate holder and support frame, as shown in Fig. 1 and Fig. 2. The solid particle can impact the target substrate with preset impact angle $\alpha_{i}$ and orientation angle $\theta_{i}$ by rotating the substrate holder and replacing the particle loader. The observation and recording system is composed of high-speed camera system, supporting light source, software and PC function terminal, which can capture the dynamic response behavior of particles and substrate in the entire impact process accurately. 


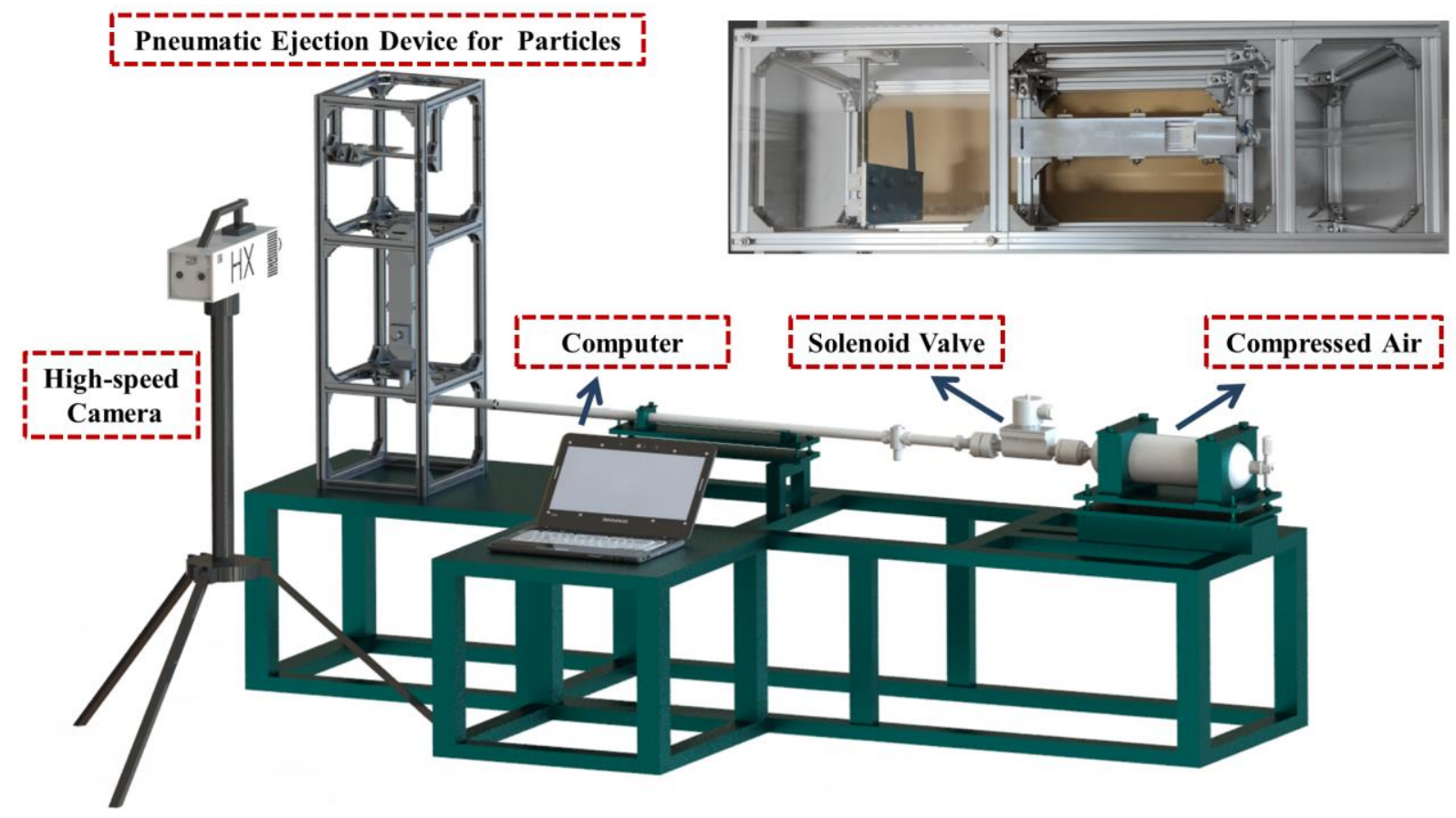

Fig.1. Pneumatic Ejection and Imaging System for Solid Particles

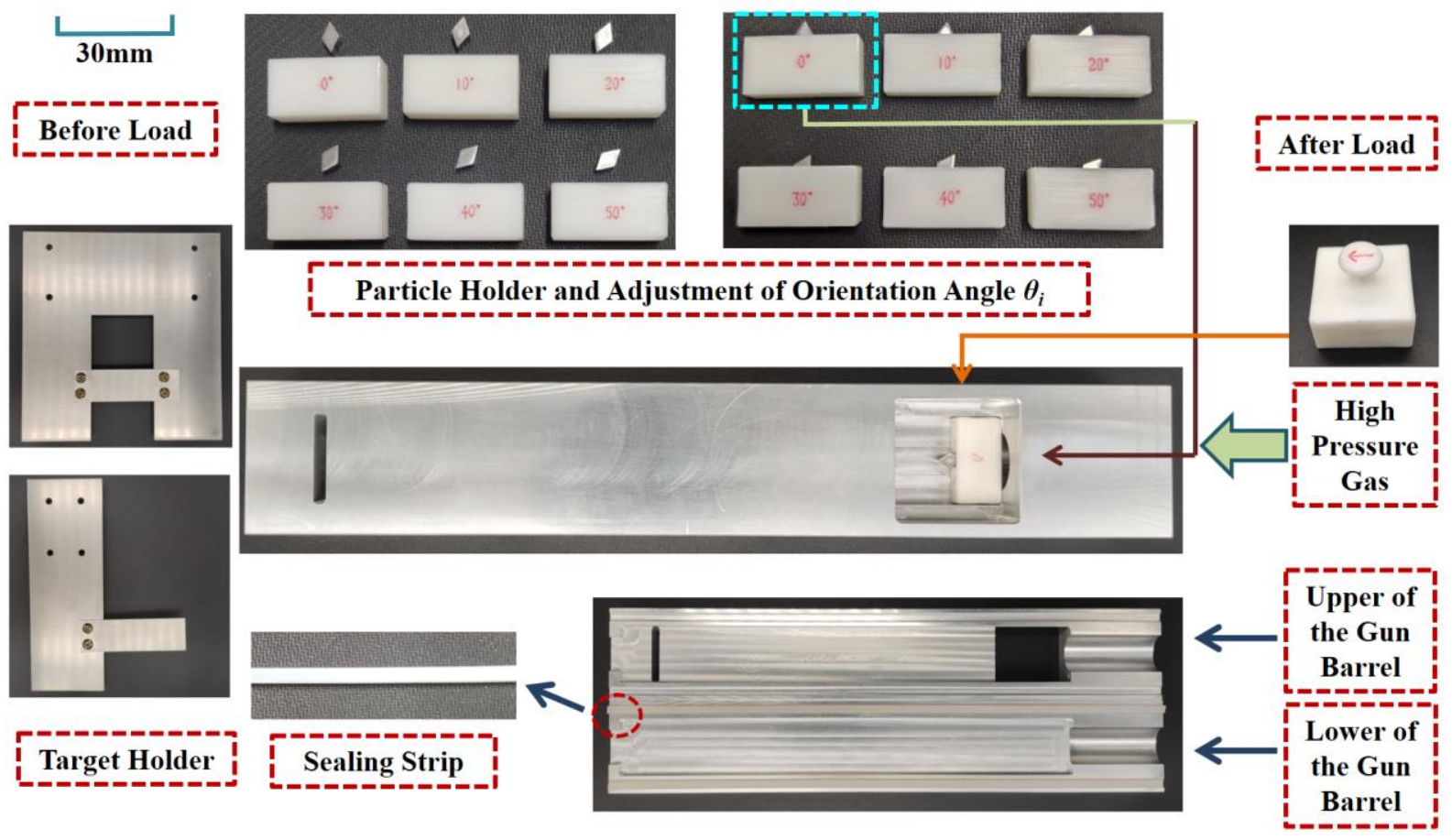

Fig.2. Adjustment of particle orientation angle $\theta_{i}$ and exhibition of variable cross-section gun barrel

\subsection{Experiment Principle}

Based on the principle of gas gun ${ }^{[18]}$, the experimental system uses compressed gas as the power source, and the compressed gas is controlled by a solenoid valve to enter the variable cross-section gun to drive the particle loader to move. After a certain period of acceleration, the particle loader and particle can obtain the 
desired velocity. At the exit of the gun barrel, the particle loader stops moving after being restrained by the stopper. The particle placed in the particle loader still have kinetic energy, fly out by inertia and impact the surface of the base substrate at the expected speed, causing erosion and damage.

The initial incident velocity of particle is determined by the initial pressure of the high pressure gas chamber $\left(p_{0}\right)$ and the length of gun barrel acceleration section $(A)$. Taking the initial velocity of particles $v_{0}$ and barrel length $A$ as the original design parameters, the maximum driving pressure of the high-pressure gas chamber is known and then other parameters of the pneumatic ejection system are determined according to the relationship between the initial pressure $p_{0}$ and $v_{0}$.

In the experiment, the process in which compressed gas accelerates the particle loader has no significant heat exchange with outside world, so it is regarded as the adiabatic process of ideal gas. The adiabatic equation ${ }^{[18]}$ is as shown in Eq. (1):

$$
p_{0} V_{0}^{\eta}=p\left(V_{0}+S_{t} x\right)^{\eta}
$$

where $p_{0}$ represents the initial pressure of the high-pressure gas chamber, $V_{0}$ represents the volume of the high-pressure gas chamber, $p$ represents the pressure of the high-pressure gas chamber at a certain time, $S_{t}$ represents the cross-sectional area of the gun barrel, $x$ represents the distance of the particle loader moves in the gun barrel at a certain time, and $\eta$ represents the gas insulation coefficient.

The motion equation ${ }^{[18]}$ of the particle loader in the gun barrel is as shown in Eq. (2):

$$
\varphi m \frac{\mathrm{d} v}{\mathrm{~d} t}=p S_{t},
$$

where $m$ represents the total mass of the particle loader and particle, $\varphi$ represents the virtual mass coefficient, which depends on the efficiency of gas work. In practical application, the value of $\varphi$ can be modified through experiments.

Eq.3 can be obtained by combining Eq. (1) and Eq. (2): 


$$
p_{0} V_{0}^{\eta}=\frac{\varphi m}{S_{\mathrm{t}}} \frac{\mathrm{d} v}{\mathrm{~d} t}\left(V_{0}+S_{t} x\right)^{\eta}
$$

Making a transformation, let $\frac{\mathrm{d} v}{\mathrm{~d} t}=\frac{\mathrm{d} v}{\mathrm{~d} x} \frac{\mathrm{d} x}{\mathrm{~d} t}$, and then substitute it into Eq. (3) can get Eq. (4):

$$
v \mathrm{~d} v=\frac{p_{0} V_{0}^{\eta} S_{t}}{\varphi m\left(V_{0}+S_{t} x\right)^{\eta}} \mathrm{d} x
$$

Integrating the two ends of Eq. (4), the relationship between the initial velocity $v_{0}$ and $p_{0}$ can be obtained as shown in Eq. (5):

$$
v_{0}^{2}=\frac{2 p_{0} V_{0}}{\varphi m(\eta-1)}\left[1-\left(\frac{V_{0}}{V_{0}+S_{t} A}\right)^{\eta-1}\right]
$$

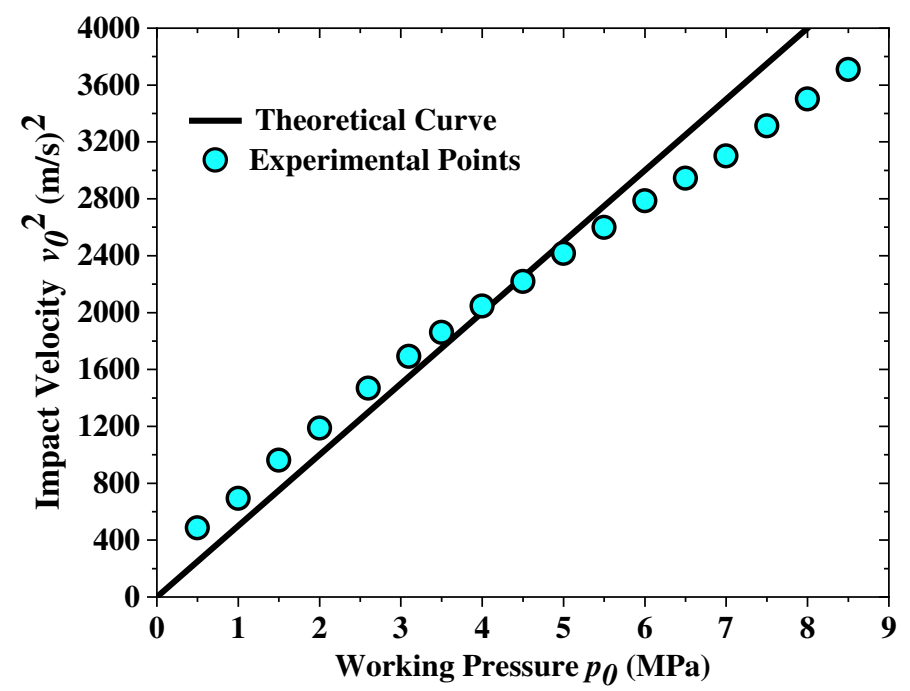

Fig.3. The relationship between initial launch velocity $v_{0}^{2}$ of gun barrel and working pressure $p_{0}$

The relationship between initial launch velocity $v_{0}^{2}$ of gun barrel and working pressure $p_{0}$ is shown in Fig. 3. Among them, $V_{0}=6.3 \mathrm{~L}, S_{t}=2.4 \times 10-4 \mathrm{~m}^{2}, m=1.079 \times 10^{-2} \mathrm{~kg}, A=0.23 \mathrm{~m}, \eta=1.4$. Taking the initial velocity $v_{0}$ and working pressure $p_{0}$ (the values shows in Fig.3) into Eq. (5), and get the average value of $\varphi$ is 20.32. Therefore, the theoretical curve of launch velocity $v_{0}$ and working pressure $p_{0}$ can be drawn as shown in Fig. 3. It should be noted that the maximum pressure of the high-pressure gas chamber is $30 \mathrm{MPa}$, so the maximum initial velocity that the system can achieve is $v_{\max }=122.5 \mathrm{~m} / \mathrm{s}$.

\section{Modeling}




\subsection{FEM-SPH Model}

The grids of Finite Element Method (FEM) elements will be distorted or twisted when dealing with transient large deformation problems, such as projectile impact and material high strain rate, which will result in unstable calculations and even interruption due to error reports. Element deletion method ${ }^{[19]}$ has been proposed to solve the above problem, while it also has obvious weakness. The deleted mesh elements destroy the integrity of the entire model, which lead to simulation results of some working conditions far away from the experimental results. The meshfree characteristic of Smoothed Particle Hydrodynamics ${ }^{[20-22]}$ (SPH) method can handle the above problem effectively, and has good applicability in dealing with high speed impact, high strain rate and other conditions. However, SPH method has some limitations, such as low computational efficiency, difficult to handle the boundary and suffer from instability under tensile loads ${ }^{[20]}$, which restrict its application prospects. In order to preserve the advantages of SPH method and ameliorate the drawbacks, FEM and SPH method based on Lagrange computing framework can be coupled to solve the problem. The advantages of FEM such as high computational efficiency, easy to handle boundary conditions combined with the SPH which can accurate simulate the transient large deformation of materials. The coupled algorithms of FEM-SPH are divided into consolidation coupled algorithm and contact coupled algorithm.

Consolidation coupled algorithm is mainly classifying into two categories ${ }^{[23]}$ : one is the fixed coupled algorithm, that is, the calculation region of FEM and SPH is determined at the initial time, and it is unchanged in the subsequent calculation process, which is suitable for the known large deformation area. The other is the adaptive coupled algorithm, which uses the FEM to calculate all the elements at the initial time, and automatically converts the elements at the large deformation area into SPH particles in the calculation process, which is suitable for the unknown large deformation region. 

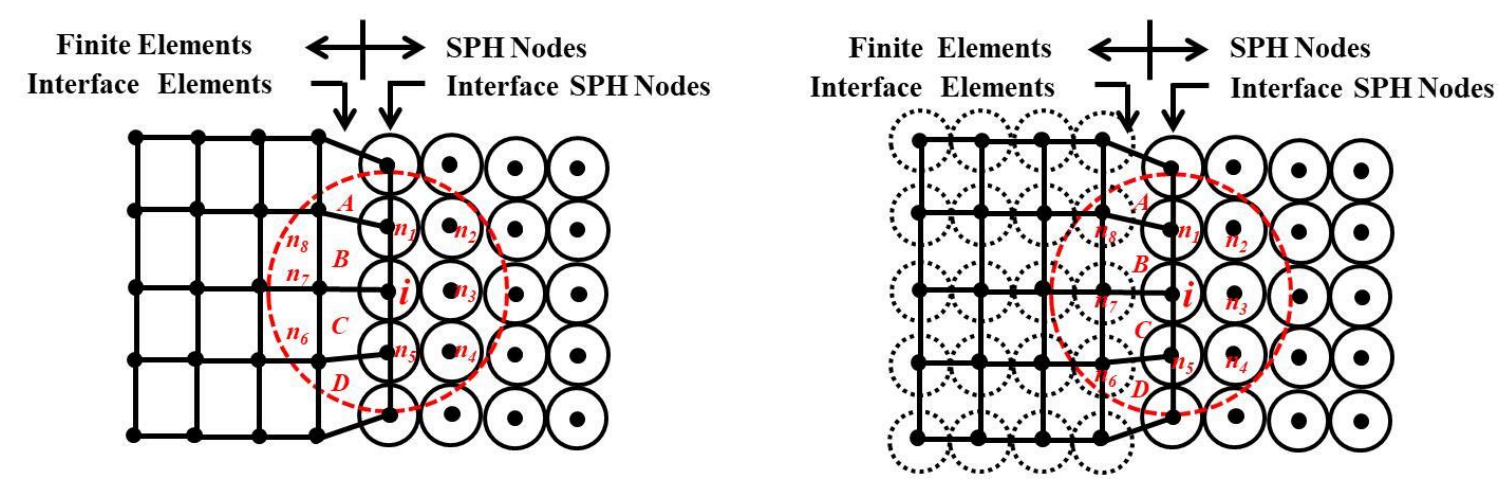

(a) SPH nodes attachment to grid without background particles [24] (b) SPH nodes attachment to grid with background particles [25]

Fig.4. SPH particles attached to finite elements

Johnson et al. ${ }^{[24]}$ proposed a fixed coupled algorithm that consolidates SPH particles onto the FE nodes through Tie constraint, and the interaction force between SPH particles and finite elements is transferred through the consolidated particles, as shown in Figure 4(a). Among them, the red broken circle represents the effective area of SPH particle on node $i$, the size of SPH particles at the interface is consistent with that of FE grids, and the masses of SPH particles at the interface comes only from smooth particles themselves, which ensures that the masses at all nodes are basically equal. This coupled mode shows that the strain and strain rate of SPH particle $i$ are only determined by $\mathrm{n}_{1} \ldots \mathrm{n}_{5}$, and the influence of the adjacent finite element nodes $\left(\mathrm{n}_{6} \ldots \mathrm{n}_{8}\right)$ in the effective area is not considered. Similarly, the force acting on SPH particle $i$ come from the particles $n_{1} \ldots n_{5}$ and the grid elements $B$ and $C$ at the interface, there is no direct or indirect force from nodes $\mathrm{n}_{6} \ldots \mathrm{n}_{8}$. This coupled method may be acceptable for many engineering applications.

Based on the research of Johnson et al. ${ }^{[24]}$, Zhang et al. ${ }^{[25]}$ added the finite element node to the SPH neighborhood search algorithm in the form of background particles (as shown in the black broken circle in Fig. 4 (b)). The background particles are only passively searched by the real SPH particles without numerical integration, and the update of data is determined by the corresponding finite element nodes. In this case, the integral of real particle $i$ includes SPH particles $n_{1} \ldots n_{5}$ and finite element nodes $n_{6} \ldots n_{8}$. This coupled method solves the problem of SPH boundary truncation and ensures the continuity of physical quantities at the interface, and applied in this paper. 
Different integration methods are adopted ${ }^{[25]}$ according to the position of the integration point and the discrete method, which are described as follows:

The domain of SPH particles far away from the coupled interface:

$$
\left\langle f\left(\boldsymbol{x}_{i}\right)\right\rangle=\sum_{j=1}^{N} \frac{m_{j}}{\rho_{j}} f\left(\boldsymbol{x}_{j}\right) W\left(\boldsymbol{x}_{i}-\boldsymbol{x}_{j}, h\right)
$$

where $f$ represents the field function of the three-dimensional coordinate vector $x, m_{j}$ and $\rho_{j}$ are the mass and density of particle $j$, respectively, $W\left(\boldsymbol{x}_{i}-\boldsymbol{x}_{j}, h\right)$ is the smooth kernel function, and $h$ represents the smooth length.

The domain of SPH particles and FEM grids at the coupled interface:

$$
\left\langle f\left(\boldsymbol{x}_{i}\right)\right\rangle=\sum_{j=1}^{N} \frac{m_{j}}{\rho_{j}} f\left(\boldsymbol{x}_{j}\right) W\left(\boldsymbol{x}_{i}-\boldsymbol{x}_{j}, h\right)+\sum_{j=1}^{N_{b}} \frac{m_{b j}}{\rho_{b j}} f\left(\boldsymbol{x}_{j}\right) W\left(\boldsymbol{x}_{i}-\boldsymbol{x}_{j}, h\right)
$$

where $m_{b j}$ and $\rho_{b j}$ are the mass and density of background particle $j$, respectively; $N_{b}$ represents the number of SPH particles and background particles in the support domain of particle $j$.

At the domain of FEM grids far away from the coupled interface:

$$
\langle f(\boldsymbol{x})\rangle=\sum_{i} N_{i}(x) f\left(x_{i}\right)
$$

where $N_{i}(x)$ represents the finite element shape function.

In order to verify the continuity of the field physical quantities at the coupled interface between the SPH particles and the FEM elements, the accuracy verify is conducted by taking stress wave propagation in a cubic bar as an example. Fixed constraint is applied on the SPH particle terminal of the FEM-SPH coupled model, and a rectangular pulse load is applied on the finite element terminal. The loading type is uniform pressure $\mathrm{P}=40 \times 10^{9} \mathrm{~Pa}$, the loading time is $0 \sim 10^{-6} \mathrm{~s}$, and the total calculation time was $14 \times 10^{-6} \mathrm{~s}$. Meanwhile, the contrasting model of FEM cubic bar is established, and the same load and boundary conditions are applied to the two models, and the stress cloud diagram in the same time step is obtained, as shown in Fig. 5. 


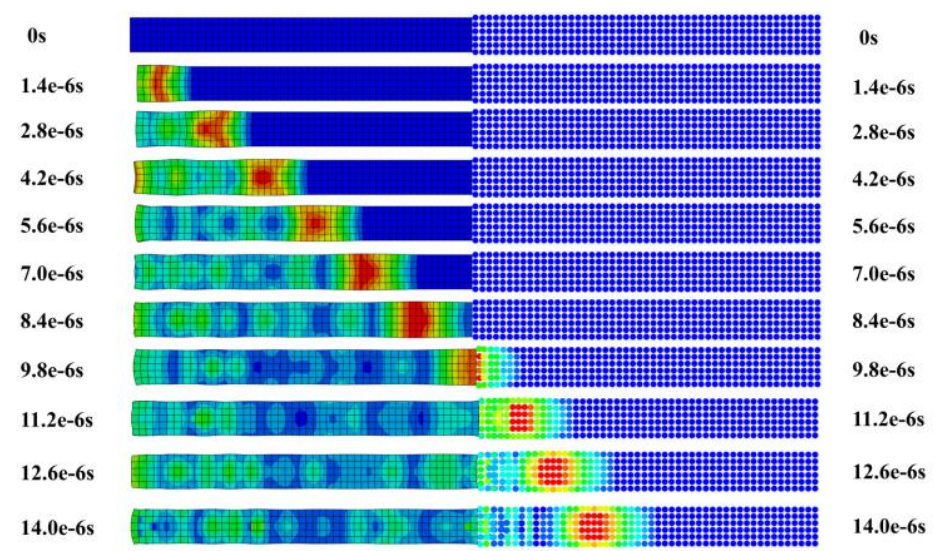

(a) Stress wave propagation of FEM-SPH coupled model

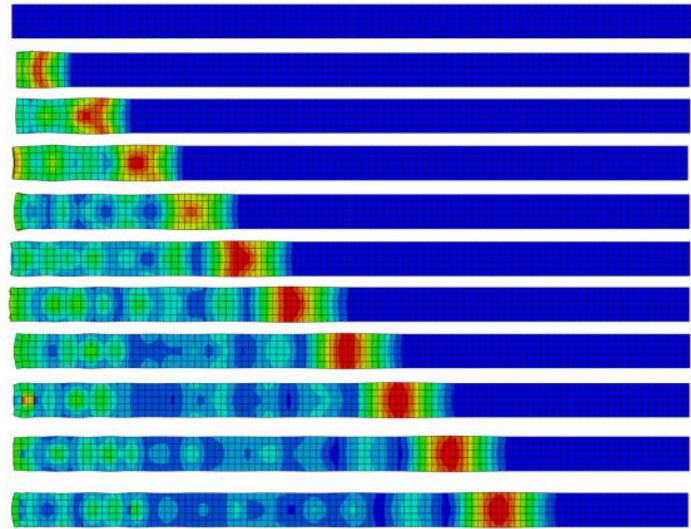

(b) Stress wave propagation of FEM model

Fig.5. Comparison of stress wave propagation between FEM-SPH model and FEM model

In order to verify the accuracy of stress field transfer quantitatively, FEM grid node 1, SPH particle 2 and FEM grid node 3 in the contrasting model are selected as reference points (as shown in Fig. 6). FEM grid node 3 is the grid node at the same position as SPH particle in the contrasting case, and the two parts of FEM mesh elements in contrasting model are also fixed together by tie constraints. The relationship between stress at the reference point and the velocity along the z-axis are plotted as shown in Fig. 7.

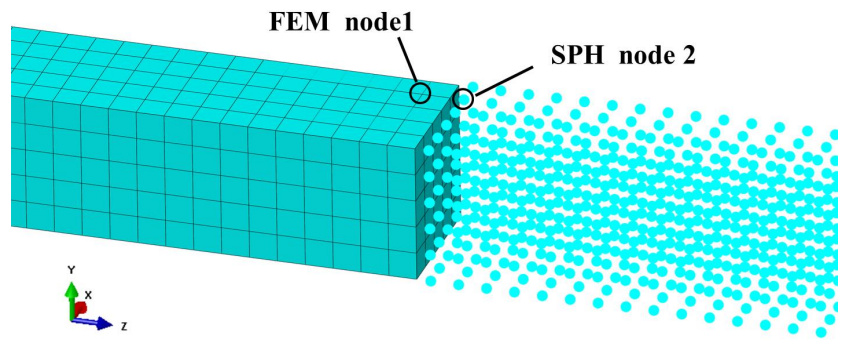

(a) Reference points on FEM-SPH coupled model

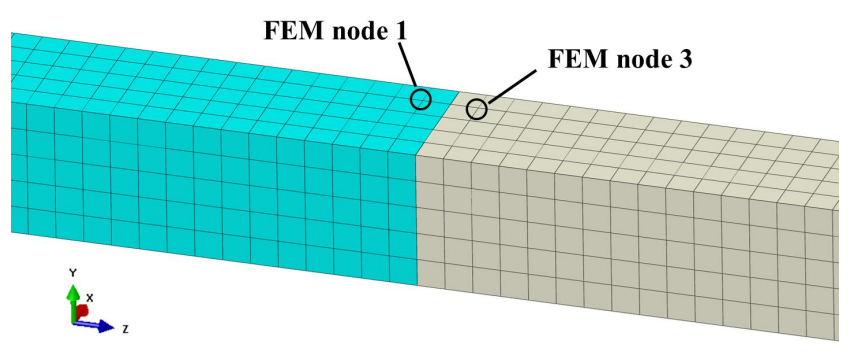

(b) Reference points on FEM model

Fig.6. Labeling of reference finite element nodes and SPH particles

From Fig. 7(a) and (b) it can be seen that the stress and velocity are both zero at the initial moment, then the plus load along the z-axis is generated and the stress wave and velocity in the z-axis direction are pass into the selected three reference points at about $\mathrm{t}=8.4 \times 10^{-6} \mathrm{~s}$. At $\mathrm{t}=9.74 \times 10^{-6} \mathrm{~s}, 9.99 \times 10^{-6} \mathrm{~s}$ and $10.20 \times 10^{-6} \mathrm{~s}$, the maximum stress of FEM grid node 1 , SPH particle 2 and FEM grid node 3 is $-27.75 \times 10^{9} \mathrm{pa},-27.95 \times 10^{9} \mathrm{pa}$ and $-27.72 \times 10^{9} \mathrm{pa}$, respectively. The maximum velocity is $\mathrm{V} z_{I}=690.97 \mathrm{~m} / \mathrm{s}, \quad \mathrm{V} z_{2}=662.78 \mathrm{~m} / \mathrm{s}$ and 
$\mathrm{V} z_{3}=692.27 \mathrm{~m} / \mathrm{s}$, respectively. Figure 7 shows that the motion of SPH particles at the coupled interface is consistent with the grid node, and is consistent with the grid node at the same position in the contrasting model, indicating that the coupled algorithm meets the continuity requirement of the physical quantity at the coupled interface.

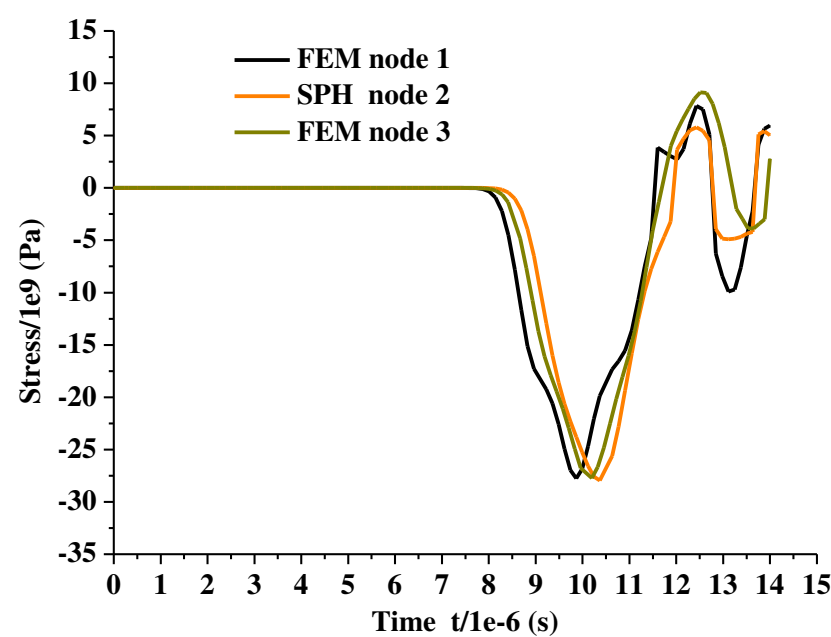

(a) The relationship of stress change with time

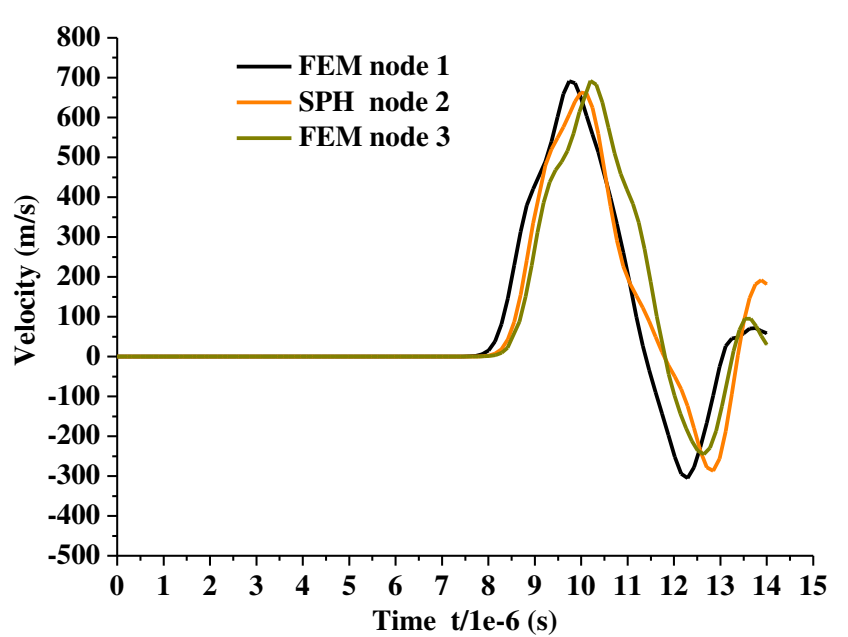

(b) The relationship of velocity change with time

Fig.7. Field variables of three reference points change with time

The contact coupled algorithm is mainly used to solve the contact problem between objects under high-speed impact load and high strain rate, with the emphasis on solving the contact force. In this process, the regions with small deformation or no deformation are discretized as FE grid elements, while the regions with large deformation are discretized as SPH particles. The principle of contact force in contact coupled algorithm is consistent with that of meshless method, that is, when the distance between FEM node and SPH particle is less than twice the smooth length $h$, corresponding contact force will be generated ${ }^{[25]}$, as shown in Fig. 8 . It should be noted that when the FEM nodes are regarded as a particle, any SPH particles in the support domain of the FEM node will generate force on the node; conversely, any FEM nodes in the SPH support domain will also generate force on the SPH particles. 


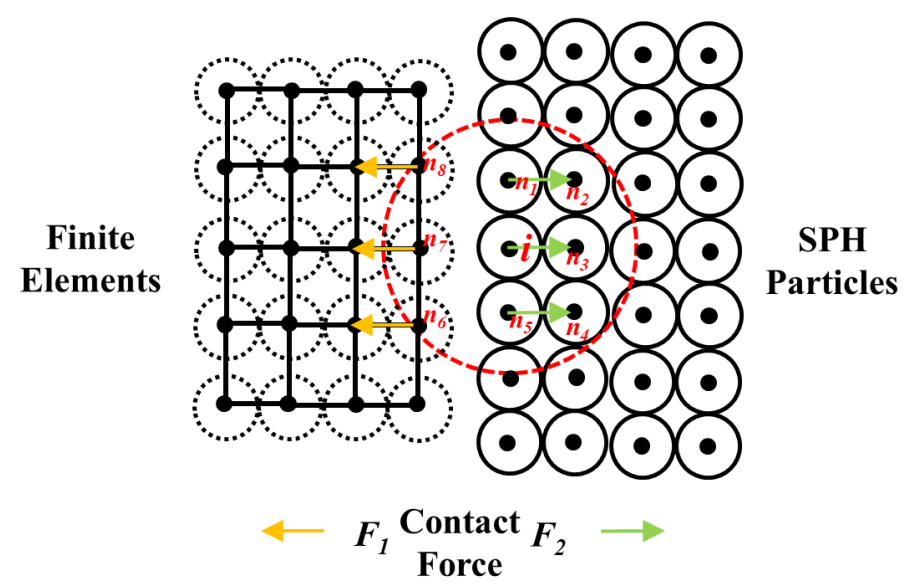

Fig.8. SPH particles contact with finite elements

The contact force equation ${ }^{[25]}$ in the approximate form of SPH particles is shown in Eq. (9)

$$
\begin{aligned}
\boldsymbol{f}_{c}\left(x_{i}\right) & =\int \boldsymbol{N}_{i}^{T} b_{c}(x) d V=b_{c}(x) \int \frac{m_{i}}{\rho_{i}} W\left(r_{i j}\right) d V=b_{c}(x) \frac{m_{i}}{\rho_{i}} \\
& =\sum_{j}^{N C O N T} \frac{m_{j}}{\rho_{j}} \frac{m_{i}}{\rho_{i}} K n \frac{W\left(r_{i j}\right)^{n-1}}{W\left(\Delta p_{\text {avg }}\right)^{n}} \nabla_{x_{i}} W\left(x_{i}-x_{j}\right),
\end{aligned}
$$

where $f_{\mathrm{c}}\left(x_{i}\right)$ represents the contact force whose direction is determined by the gradient of SPH smooth kernel function; $\boldsymbol{N}_{i}^{\mathrm{T}}$ represents the shape function matrix, and the shape function of particle $i$ in the SPH method is shown in Eq. (10)

$$
N_{i j}=m_{j} \mathrm{~g} W_{j}\left(x_{i}\right) / \rho_{j} \mathrm{~g} \sum_{j=1}^{N_{p}} W_{j}\left(x_{i}\right),
$$

where $j$ is the particle in the support region of $i$, and $N_{p}$ is the number of neighboring particles in the support region of $i ; b_{c}(x)$ represents the body force of the contact part; NCONT represents the number of adjacent particles from different contact parts in the support domain of particle $i$ (FEM nodes are regarded as SPH particles). For particle $i$ in Fig. 8 , corresponding to NCONT $=3$, that is, $n_{6}, n_{7}, n_{8}$ nodes will affect the contact force of particle $i . m_{j}$ and $\rho_{j}$ represent the mass and density of particle $j$, respectively. $W$ represents the smooth kernel function, when $x_{A}$ and $x_{B}$ belong to the same target, $W\left(x_{A}-x_{B}\right)=0 ; r_{i j}$ is the distance between particles, and $\Delta p_{\text {avg }}$ is the average value of the smooth length between particles; $K$ and $n$ are user-defined parameters, where $K$ is similar to the contact stiffness in the finite element contact algorithm, and is related to the material 
properties and contact speed.

When applying contact force on SPH particles, it is necessary to modify the SPH momentum equation and take the contact force into consideration. The modified momentum equation for the model is given by

$$
\frac{\partial \boldsymbol{v}_{i}^{\alpha}}{\partial t}=\sum_{j=1}^{N} m_{j}\left(\frac{\sigma_{i}^{\alpha \beta}}{\rho_{i}^{2}}+\frac{\sigma_{j}^{\alpha \beta}}{\rho_{j}^{2}}+\Pi_{i j}\right) \frac{\partial W_{i j}}{\partial x_{i}^{\beta}}-\frac{f_{c}^{\alpha}\left(x_{i}\right)}{m_{i}},
$$

where $\sigma$ represents the total stress tensor, which is composed of isotropic pressure $p$ and viscous stress $\tau$, that is, $\sigma^{\alpha \beta}=-p \delta^{\alpha \beta}+\tau^{\alpha \beta}$. $\Pi_{i j}$ represents artificial viscosity to eliminate unnecessary non-physical oscillation.

The dynamic equation ${ }^{[25]}$ of finite element is shown in Eq. (12)

$$
M \&(t)+C \iota \&(t)+K u(t)=Q(t)
$$

where $u(t)$ and $\ddot{u}(t)$ represent nodal displacement and nodal acceleration, respectively; $\boldsymbol{M}, \boldsymbol{C}, \boldsymbol{K}$ and $\boldsymbol{Q}(t)$ represent the mass matrix, damping matrix, stiffness matrix and nodal load vector of the system, respectively.

When applying contact force on the FEM node, the node load $\boldsymbol{Q}(t)$ in the Eq. (12) should be replaced by the external force $\boldsymbol{f}_{\mathrm{c}}\left(x_{i}\right)$, that is,

$$
\boldsymbol{M} \&(t)+\boldsymbol{C} \&(t)+\boldsymbol{K} u(t)=\boldsymbol{f}_{c}(t)
$$

In this paper, the process of rhomboid shaped particle impacting cantilever beam and fixed-fixed beam is studied. The large deformation region is known, and the stiffness of particle is far more than the substrate material. Therefore, the consolidation coupled algorithm and contact coupled algorithm are selected. FEM-SPH coupled models of rhomboid-shaped particles impacting A16061-T6 thin plate (cantilever beam and fixed-fixed beam) are shown in Fig. 9. The region where elastic large deformation and plastic damage occurs of the thin plate is discretized as SPH particles, the rhomboid-shaped particles and fixed terminal far away from the impact area are discretized as FEM grids.

The size of the thin plate model involved in the calculation is shown in Fig. 9(a) and (b). Cantilever beam is restrained at one terminal and the fixed-fixed beam is restrained at both terminals. The space between SPH 
particles is $20 \mu \mathrm{m}$. General contact between particles and material substrate is defined and the dynamic friction coefficient is 0.1 to improve the consistency between simulation and experiments results. In the simulation, the incident particle is constrained as rigid body to improve calculation efficiency.

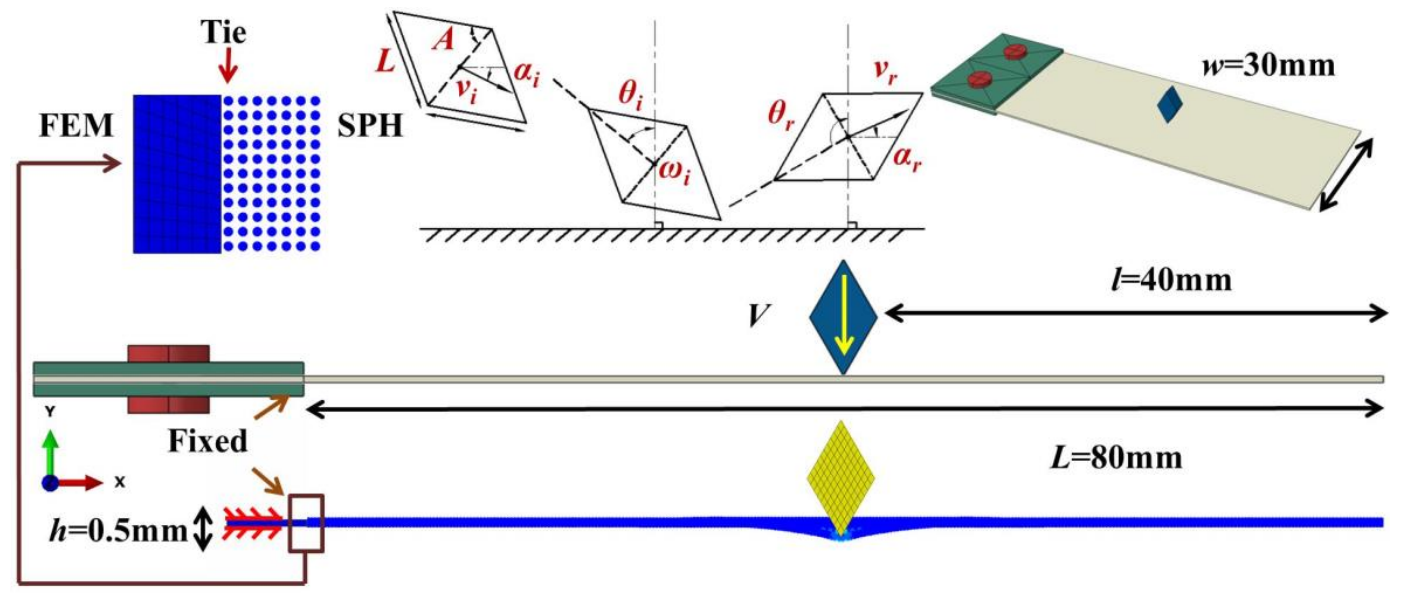

(a) Rhomboid shaped particle impacting cantilever beam

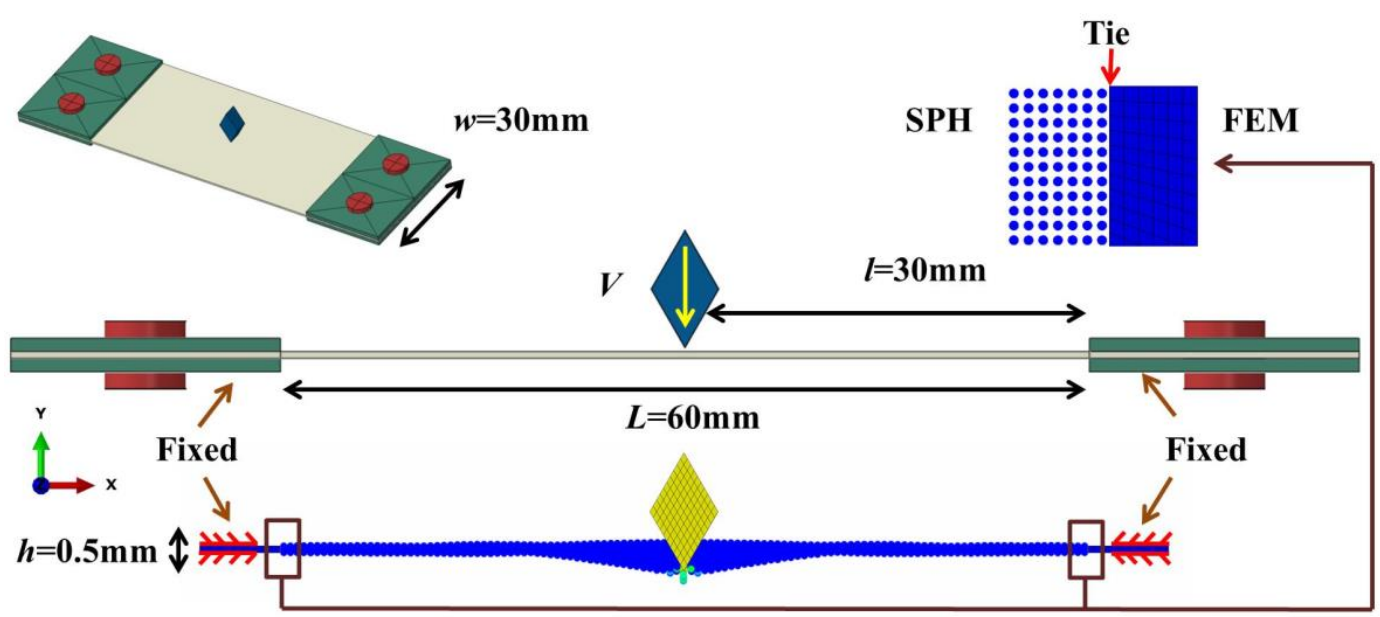

(b) Rhomboid shaped particle impacting fixed-fixed beam

Fig.9. Coupled FEM-SPH numerical model of rhomboid-shaped particle impacts A16061-T6 thin sheet

\subsection{Material Model}

\section{Johnson-Cook model for target materials}

Johnson-Cook constitutive model was proposed by Johnson and Cook ${ }^{[26]}$ to simulate the high-speed strain behavior of ductile materials under instantaneous strong load impact, which consist of two parts: strength and damage. The model considers the effects of strain hardening, viscous strain-rate and thermal 
softening, and is most widely used in the simulation of strong load, large strain and high temperature.

\section{(a) Strength}

The formulation of Johnson-Cook plastic model is shown in Eq. (14), where the flow stress $\sigma_{f}$ is a function of the equivalent plastic strain $\varepsilon$, the equivalent plastic strain rate \& and the temperature $\mathrm{T}$.

$$
\sigma_{f}=\left(A+B \varepsilon^{n}\right)(1+C \ln \&)\left(1-T^{* m}\right)
$$

Where the normalized equivalent strain rate $\&$ and temperature $T^{*}$ are defined as Equation (15) and (16)

$$
\begin{gathered}
\mathscr{\&}=\& \varepsilon_{0} \\
T^{*}=T-T_{r} / T_{m}-T_{r}
\end{gathered}
$$

Where $\varepsilon_{0}$ is the reference plastic strain rate, $T, T_{m}$, and $T_{r}$ are the instantaneous, melting, and reference temperatures, respectively. The parameters $A, B$ and $n$ are material constants describing the initial yield and strain hardening behavior of the material. The parameters $C$ and $m$ describing the material strain rate hardening and thermal softening sensitivity, respectively.

\section{(b) Damage}

Particles impact on the surface of metal at a high velocity may cause permanent damage. The Johnson-Cook failure model was introduced to simulate the failure behavior of metal materials. When the damage parameter $D_{i}=1^{[27]}$ in Eq. (17), the affected SPH particle fails and is therefore removed from the system.

$$
D=\sum \Delta \varepsilon / \varepsilon_{f}
$$

Where $\Delta \varepsilon$ is the plastic strain increment of SPH particle during a cycle of integration, and $\varepsilon_{f}$ is the failure strain of material, the formulation of which shown in Eq. (18) ${ }^{[27]}$. This damage model considers the effect of stress triaxiality (first term), strain rate hardening (second term) and thermal softening (third term).

$$
\varepsilon_{f}=\left(d_{1}+d_{2} e^{d_{3}\left(\sigma_{p} / \sigma_{e}\right)}\right)\left(1+d_{4} \ln \&\right)\left(1+d_{5} T^{*}\right)
$$

Where $\sigma_{p}$ is the principal stress and $\sigma_{e}$ is the effective stress, $d_{1}, d_{2}, d_{3}, d_{4}$ and $d_{5}$ are five material 
constants of Johnson-Cook failure model, which are obtained by experimental tests.

In this paper, the erosion behavior of A16061-T6 material has been studied. The material parameters of $\mathrm{J}-\mathrm{C}$ plastic model and damage model ${ }^{[7]}$ are shown in Table 1.

Table1. Johnson-Cook materials parameters for Al-6061-T6 metal

\begin{tabular}{lcc}
\hline Material Properties & Symbol & Al-6061T6 $6^{[7]}$ \\
\hline Density $/\left(\mathrm{kg} / \mathrm{m}^{3}\right)$ & $\rho_{0}$ & 2800 \\
Shear modulus $/ \mathrm{GPa}$ & $\mathrm{G}$ & 26 \\
Poisson's ratio & $\mathrm{v}$ & 0.33 \\
Melting temperature/K & $\mathrm{T}_{\text {melting }}$ & 925 \\
Reference temperature/K & $\mathrm{T}_{\mathrm{ref}}$ & 293 \\
Specific heat/[J/(kg $\cdot \mathrm{K})]$ & $\mathrm{C}_{\mathrm{P}}$ & 885 \\
Strength Const. & & \\
$\mathrm{J}-\mathrm{C}$ yield strength/MPa & $\mathrm{A}$ & 335 \\
$\mathrm{~J}-\mathrm{C}$ hardening coefficient/MPa & $\mathrm{B}$ & 85 \\
$\mathrm{~J}-\mathrm{C}$ strain hardening coefficient & $\mathrm{n}$ & 0.11 \\
$\mathrm{~J}-\mathrm{C}$ strain rate constant & $\mathrm{C}$ & 0.012 \\
$\mathrm{~J}-\mathrm{C}$ strain rate sensitivity index & $\mathrm{m}$ & 1.6 \\
Damage Const. & & \\
J-C damage constant & $d_{l}$ & -0.77 \\
J-C damage constant & $d_{2}$ & 1.45 \\
J-C damage constant & $d_{3}$ & -0.47 \\
J-C damage constant & $d_{4}$ & 0 \\
J-C damage constant & $d_{5}$ & 1.6 \\
Pressure Const. & & 5240 \\
Elastic bulk wave velocity & $\mathrm{C}_{0}(\mathrm{~m} / \mathrm{s})$ & 1.4 \\
Slope in $U s$ vs $U p$ diagram & $\mathrm{S}_{\mathrm{a}}$ & 1.97 \\
Grüneisen coefficient & $\gamma_{0}$ & \\
\hline
\end{tabular}

\section{Equation of state}

The erosion wear of solid particle impact on metal surface at high velocity is an almost instantaneous large deformation process, where the dynamic load of particle contact to the substrate far exceeds the tensile strength of target. Equation of State (EOS) can completely describe the dynamic response process of the impacted material by defining the relationship between hydrostatic pressure, the local density and local specific energy. Mie-Gruneisen equation of state ${ }^{[28]}$ was frequently used in impact collisions, as shown in Eq. (19).

$$
P=\frac{\rho_{0} C_{0}^{2} \eta\left[1+\left(1-\Gamma_{0} / 2\right) \eta\right]}{\left[1-\left(S_{a}-1\right) \eta\right]}+\rho_{0} \Gamma_{0} e
$$

Where $P$ is the actual pressure, $\rho_{0}$ is the initial density, $C_{0}$ is the volume wave velocity, $\Gamma_{0}$ is the 
Grüneisen coefficient, $S_{a}$ is the slope parameter, $\eta=\rho / \rho_{0^{-}} 1$ is represent the compression, and $e$ is the internal energy.

For most solids and fluids, the particle velocity $(U p)$ and shock velocity $(U s)$ follow a simple linear relationship under lower shock load, ie $U s=C_{0}+s U p$. The parameters of particle velocity $(U p)$ and shock velocity $(U s)$ are measured by dynamic load experiments, which can be used to solve the above Equation, and the parameters of EOS are determined as shown in Table 1. However, under ultra-high-speed shock load, there is a nonlinear relationship between particle velocity $(U p)$ and shock velocity $(U s)$, which needs further correction and not discussed here.

\section{Model Validation}

The comparison of experimental and simulated results of rhomboid-shaped particle impacting the center of the cantilever beam and the fixed-fixed beam are as shown in Fig.10. It can be seen that the simulation predicted results are in good agreement with the experimental measured results, and the FEM-SPH coupled model can accurately capture the deformation characteristic of thin cantilever beam and fixed-fixed beam impacted by particles. For example, when rhomboid-shaped particle impacts the center of cantilever beam (Fig. 10(a)), plastic deformation first occurs at the position where the substrate and particle tip contact and the corresponding plastic crater generated as shown in Fig. 11(a). Meanwhile, elastic deformation occurs at the impact position of the cantilever beam and gradually reaches the maximum deflection value $\gamma$ (Fig. 12(a)). With the recovery of elastic deformation, the free terminal of the cantilever beam begins to move and rebound after reaching the maximum value $\delta$ (as shown in Fig. 12(a)). After the rebound, the free terminal reaches the reverse maximum deflection $\zeta$ and then rebounds again. Due to the factors of air resistance and gravity, the value $\zeta$ is slightly lower than $\delta$. As the above process circulating, the vibration amplitude of the cantilever free terminal decreases gradually. The particle has obvious forward rotation (clockwise) after impact, resulting in secondary impact phenomenon and forming secondary crater, as shown in Fig. 10(a) and 11(a). It is worth 
noting that with the increase of the incident velocity, the plastic crater profile of the cantilever will be breakdown, while the vibration response characteristics of the cantilever beam are basically consistent with those under the low incident velocity, as shown in Fig. 13(a).
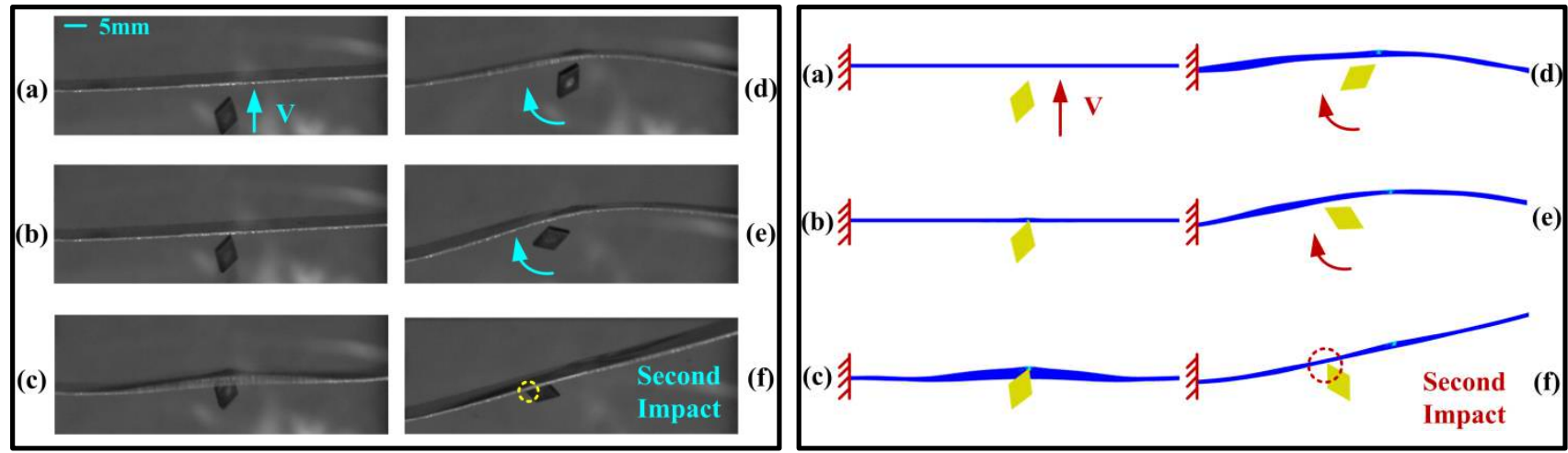

(a) Rhomboid-shaped particle impacting on cantilever beam: $\mathrm{V}=36.75 \mathrm{~m} / \mathrm{s}, \alpha_{i}=90^{\circ}, \theta_{i}=-16^{\circ}$
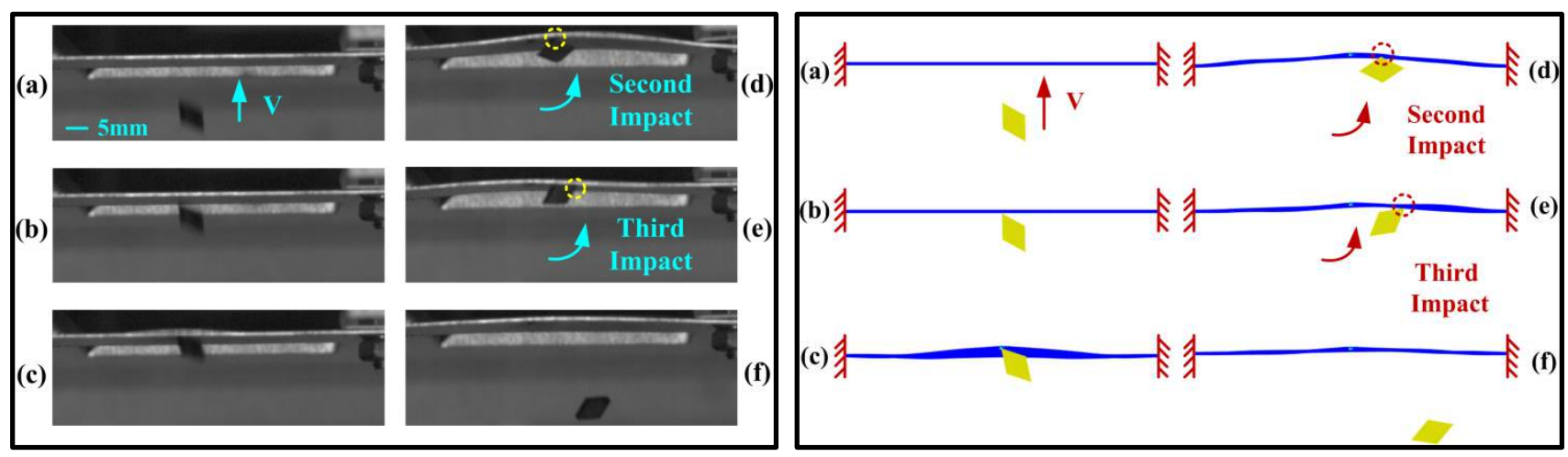

(b) Rhomboid-shaped particle impacting on fixed-fixed-beam: $\mathrm{V}=34.75 \mathrm{~m} / \mathrm{s}, \alpha_{i}=90^{\circ}, \theta_{i}=31^{\circ}$

Fig.10. Comparison of experimental and simulated results of rhomboid-shaped particle impacting on Al6061-T6 thin plate

When rhomboid-shaped particle impacts the center of fixed-fixed beam whose two terminals are restrained (Fig. 10(b)), plastic deformation first occurs at the impact position and the plastic crater formed as shown in Fig. 11(b). In the meantime, a portion of initial kinetic energy of incident particle is transformed into the elastic deformation of the fixed-fixed beam. When the maximum deflection $\gamma^{\prime}$ (as shown in Fig. 12(b)) is reached at the impact position, the fixed-fixed beam rebounds, and after the rebound reaches the reverse maximum deflection $\zeta^{\prime}$, it rebounds again, which is similar to the vibration behavior of the free terminal of the cantilever beam. Similarly, particle after impact has obvious backward rotation (anticlockwise) and three 
impacts occurred, as shown in Fig. 10(b) and 11(b). It should be noticed that with the increase of incident velocity, the plastic crater profile of the fixed-fixed beam will be breakthrough, and the particle will be embedded in the thin plate in some cases. In addition, plastic bending occurs at both terminals of the fixed-fixed beam under the strong impact of particle, the reverse rebound ability at the impact point is weakened, and even cannot be returned to the initial horizontal position, as shown in Fig. 13(b).

From Fig. 12 and 13 it can be seen that under the same incident conditions, the maximum deflection value at the impact position of the cantilever beam is greater than that at the same position of the fixed-fixed beam, indicating that the proportion of the kinetic energy converted into elastic potential energy of the cantilever beam after impact is significantly higher than that of the fixed-fixed beam under the same incident conditions.
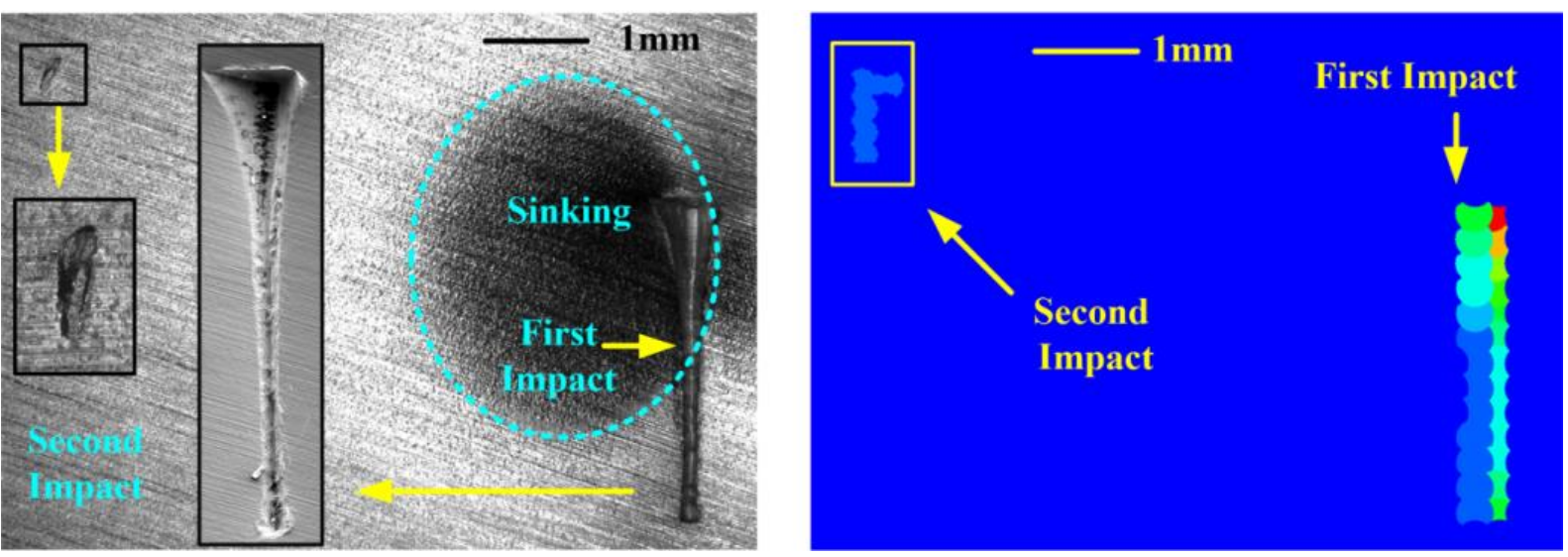

(a) Contour of craters on cantilever beam formed by rhomboid-shaped particle
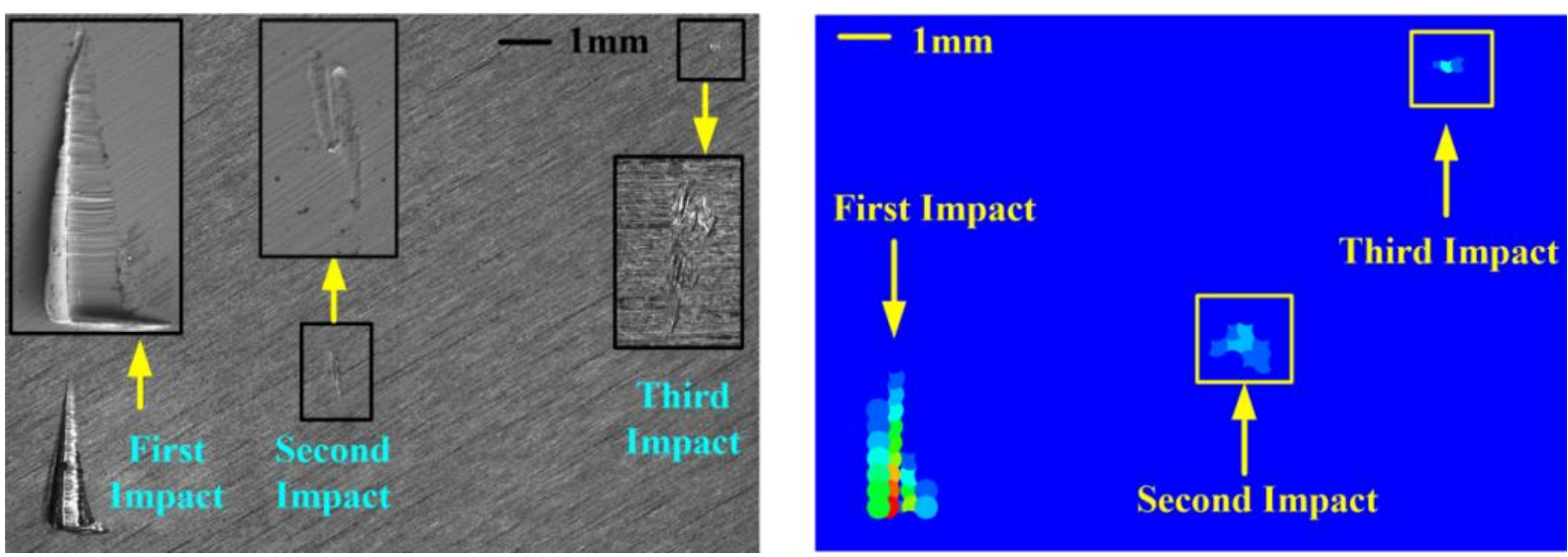

(b) Contour of craters on fixed-fixed beam formed by rhomboid-shaped particle

Fig.11. Comparison of experimental and simulated results of craters profile formed by rhomboid-shaped particle 


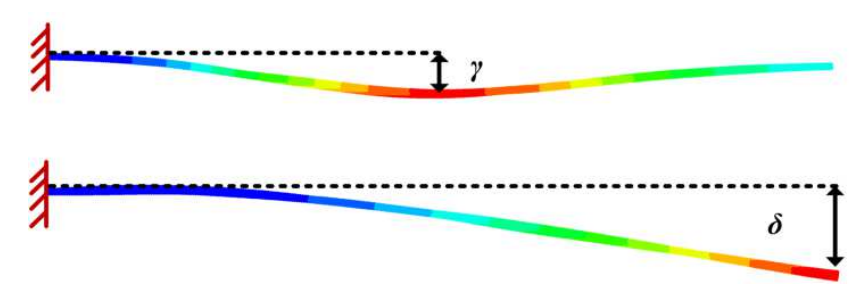

(a) Deformation deflection diagram of cantilever beam

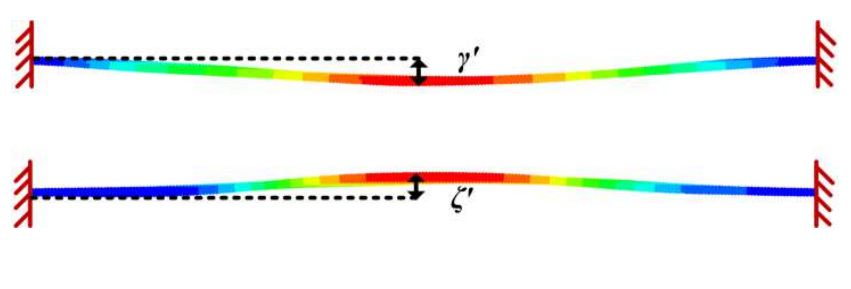

(b) Deformation deflection diagram of fixed-fixed-beam

Fig.12. Deflection diagram of cantilever beam and fixed-fixed beam after being impacted by particles $\left(\mathrm{V}=20 \mathrm{~m} / \mathrm{s}, \alpha_{i}=90^{\circ}, \theta_{i}=0^{\circ}\right)$

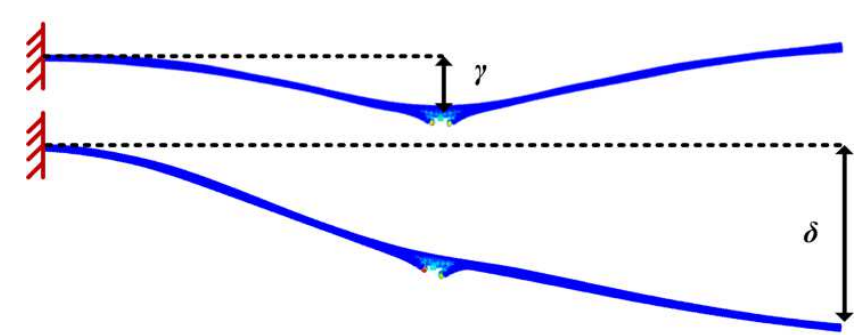

(a) Plastic bending and breakthrough of cantilever beam

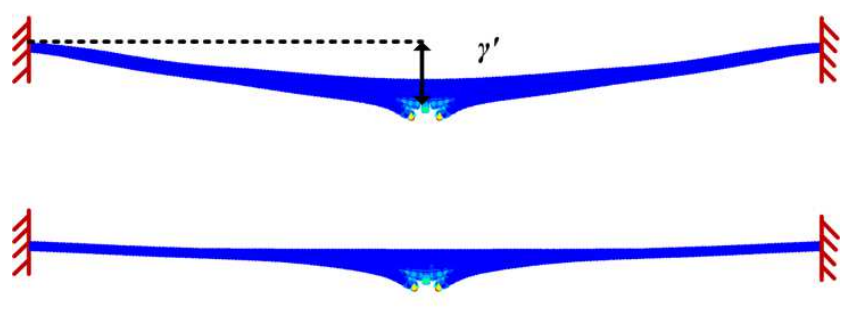

(b) Plastic bending and breakthrough of fixed-fixed beam

Fig.13. Plastic bending and breakthrough of thin plate (cantilever beam \& fixed-fixed beam) after impacting by rhomboid-shaped particle under medium-high velocity $\left(\mathrm{V}=60 \mathrm{~m} / \mathrm{s}, \alpha_{i}=90^{\circ}, \theta_{i}=0^{\circ}\right)$

By contrast it is found that under low-speed incident conditions, the thin cantilever beam substrate only undergoes elastic deformation after being impacted by particles, and does not generate plastic deformation, in consequence the FEM model and the FEM-SPH coupled model can capture the same experimental phenomenon. However, the efficiency of FEM-SPH model is lower than that of FEM model due to its time-consuming in searching adjacent particles and optimizing the smooth length. With the increase of the incident velocity, there is a threshold velocity $v_{l}$, which results in permanent plastic craters forming on the surface of the material substrate. The threshold velocity is related to the size parameters of the thin plate and the impact position of the particles. With the further increase of the incident velocity, there is a threshold velocity v', which makes the particle tip breakthrough the target substrate. The FEM method cannot accurately solve the large deformation and grid distortion conditions. The phenomenon of substrate breakdown impacted by particle can only be simulated by the element deletion method, and cannot accurately capture the mode III 
cracking phenomenon ${ }^{[29]}$ of the substrate at the moment of breakdown. The FEM-SPH coupled model can accurately capture the phenomenon of particle breakdown and substrate cracking, and can precisely obtain the specific value of the critical velocity $v^{\prime}$, which is also related to the size parameters of thin plate and the impact position of particle.
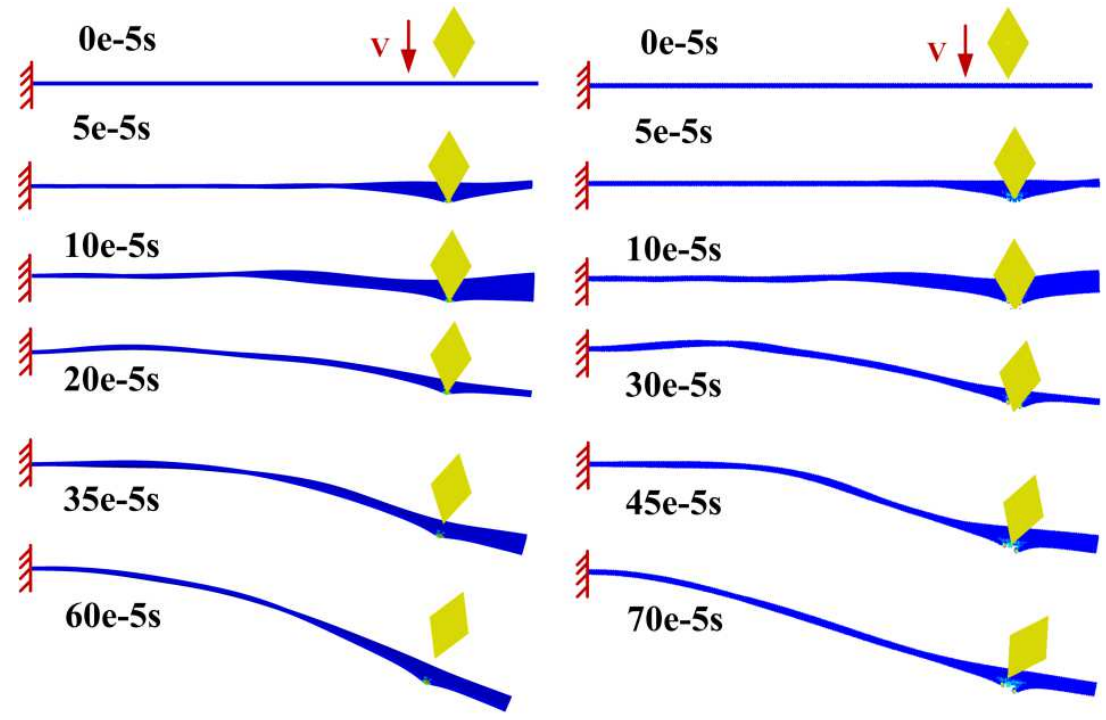

(a) Simulated results of FEM model

(b) Simulated results of FEM-SPH coupled model

Fig.14. Comparison and verification of different models to capture substrate deformation under medium-high velocity impact

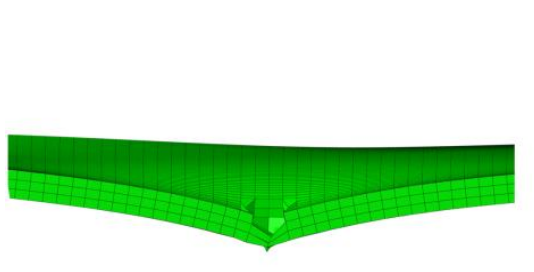

(a) Crater predicted by FEM model conditions $\left(\mathrm{V}=60 \mathrm{~m} / \mathrm{s}, \alpha_{i}=90^{\circ}, \theta_{i}=0^{\circ}\right)$

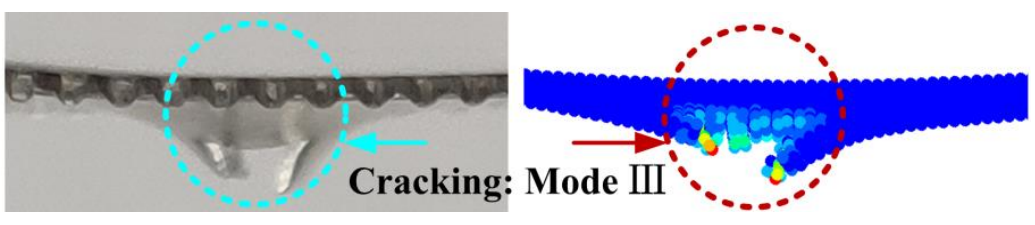

(b) Crater from experiments

(c) Crater predicted by FEM-SPH coupled model

Fig.15. Comparison and verification of different models to capture substrate breakdown under medium-high velocity impact conditions

Fig. 14 shows the dynamic deformation process of rhomboid-shaped particle impacting the cantilever beam captured by FEM model and FEM-SPH coupled model under the medium-high speed impact condition $\left(\mathrm{V}=60 \mathrm{~m} / \mathrm{s}, \alpha_{i}=90^{\circ}, \theta_{i}=0^{\circ}\right)$, which the cantilever beam of the substrate has been breakdown. Fig. 15 shows the crater contour of the substrate after breakdown predicted by the corresponding two models in Fig. 14. The FEM model achieves the effect of breakdown by deleting the large deformation grid elements, while the 
FEM-SPH coupled model can reproduce the particle breakdown process without particle deletion, and can accurately capture the micro phenomena such as mode III cracking of the substrate.

\section{Results and discussion}

\subsection{Effects of substrate width $w$}

The width $w$ of cantilever beam is one of the important factors affecting the maximum deflection and crater morphology of the substrate. Taking rhomboid-shaped particle impacting the middle position of cantilever beam $(L=60 \mathrm{~mm}, h=0.5 \mathrm{~mm})$ vertically as an example, Fig. 16 shows the variation of maximum deflection $\gamma$ at the impact position of cantilever beam and maximum deflection $\delta$ at the free terminal with the width $w$ under the same incident conditions. It can be seen from the Fig. 16 that with the increase of the cantilever beam width $w$, the maximum deflection $\gamma$ at the impact position and the maximum deflection $\delta$ at the free terminal shows a tendency of gradual decrease, and the decrement gradually weakening.

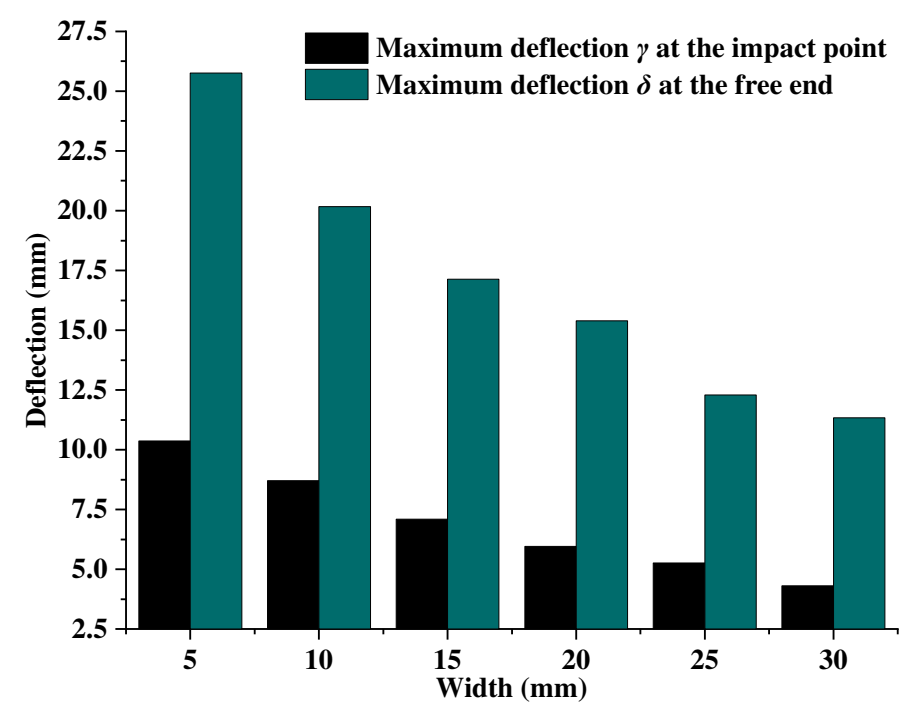

Fig. 16 Variation of maximum deflection $\gamma$ and $\delta$ of cantilever beam with beam width w $\left(\mathrm{V}=30 \mathrm{~m} / \mathrm{s}, \alpha_{i}=90^{\circ}, \theta_{i}=0^{\circ}, L=60 \mathrm{~mm}, h=0.5 \mathrm{~mm}\right)$

Fig. 17 shows the deformation behavior of cantilever beams $(L=60 \mathrm{~mm}, h=0.5 \mathrm{~mm})$ with width $w$ of $10 \mathrm{~mm}$ and $30 \mathrm{~mm}$ under the same impact condition. It can be seen that the elastic deformation of cantilever beam width of $10 \mathrm{~mm}$ is significantly greater than that of cantilever beam with width of $30 \mathrm{~mm}$ under the same impact condition. One of the reasons may be affected by the mass of the substrate. For the same length and 
thickness of the substrate, the smaller the width $w$, the smaller the mass of the substrate, and the smaller its own inertia, so the response speed of elastic deformation after impact by particle is significantly faster. In this way, the initial kinetic energy of the particles is more transformed into the elastic potential energy of the substrate, which results in the elastic deformation of the cantilever beam is significantly increased (as shown in Fig. 17 (a)), while the plastic deformation extent is low, so the crater contour is not obvious (Fig. 18 (a)). Similarly, the greater the width $w$, the greater the inertia of the substrate and the response time of elastic deformation after impact by particle is obviously delayed. In this way, the initial kinetic energy of the particles is more transformed into the plastic strain energy of the substrate, and the deformation extent of the crater contour is enhanced (as shown in Fig. 18 (b)), while the corresponding elastic deformation extent is reduced (Fig. 17 (b)). Another reason may be affected by the boundary effect. For the same length and thickness of the substrate, the smaller the width $w$, the more the substrate tends to be "slender strip". Under the same impact condition, the fixed terminal of cantilever beam has weaker resistance to external load and is more prone to plastic bending deformation. Therefore, it is observed that the cantilever beam with smaller width has greater deformation than that with larger width.

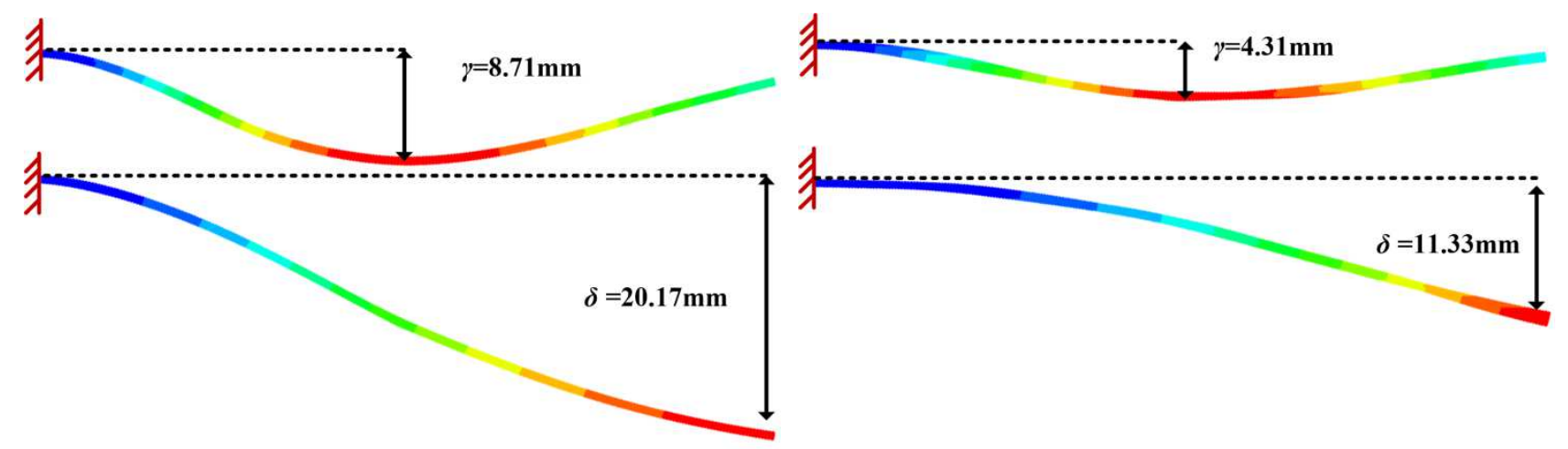

(a) The width of cantilever beam: $w=10 \mathrm{~mm}$

(b) The width of cantilever beam: $w=30 \mathrm{~mm}$

Fig.17. Deformation behavior of cantilever beams with different widths under the same impact condition $\left(\mathrm{V}=30 \mathrm{~m} / \mathrm{s}, \alpha_{i}=90^{\circ}, \theta_{i}=0^{\circ}\right)$ 

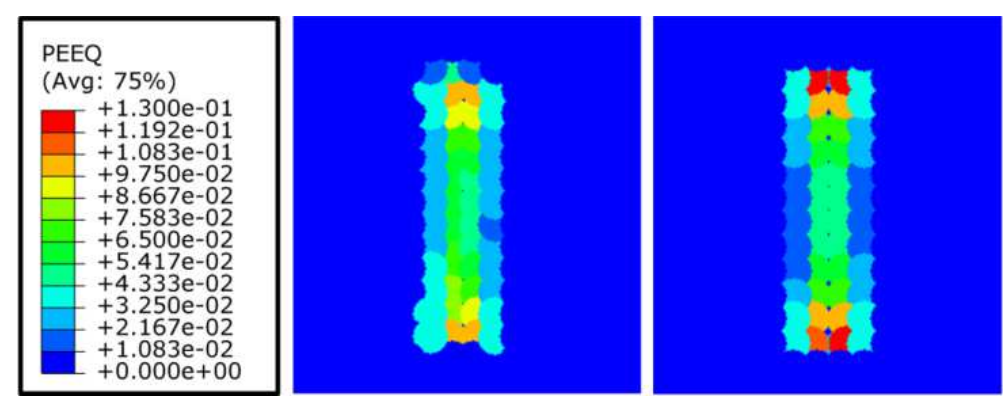

(a) The width of cantilever beam: $w=10 \mathrm{~mm}$

(b) The width of cantilever beam: $w=30 \mathrm{~mm}$

Fig.18. Crater contour of cantilever beams with different widths under the same impact condition $\left(\mathrm{V}=30 \mathrm{~m} / \mathrm{s}, \alpha_{i}=90^{\circ} \theta_{i}=0^{\circ}\right)$

\subsection{Effects of impact velocity $V$}

The incident velocity $V$ of particle is one of the important factors affecting the maximum deflection and crater morphology of the substrate. Taking rhomboid-shaped particle impacting the middle position of cantilever beam $(L=60 \mathrm{~mm}, w=30 \mathrm{~mm}, h=0.5 \mathrm{~mm})$ vertically as an example, elastic deformation and slight plastic deformation occur at the impact point under low speed conditions $(V=20 \mathrm{~m} / \mathrm{s})$, and the contour of the crater is not obvious. With the increase of incident velocity, the extent of elastic deformation and plastic deformation at the impact position is obviously enhanced. When the velocity $V=50 \mathrm{~m} / \mathrm{s}$, the plastic crater is breakdown and the chip separation phenomenon appears, as shown in Fig. 19. It is worth noting that the threshold value of breakdown velocity is related to the size of the cantilever beam. Taking the beam width $w$ discussed in section 5.1 as the breakthrough point, the length $L$ and thickness $h$ of the cantilever beam remain unchanged. With the increase of the cantilever beam width $w$, the threshold value of breakdown velocity decreases gradually. Fig. 20 (a) and (b) show the deformation behavior of the cantilever beam with the width of $10 \mathrm{~mm}$ and $30 \mathrm{~mm}$ respectively after being impacted by rhomboid-shaped particles under the same incident condition $\left(V=60 \mathrm{~m} / \mathrm{s}, \alpha_{i}=90^{\circ}, \theta_{i}=0^{\circ}\right)$. The deformation extent of the cantilever beam with $w=10 \mathrm{~mm}$ is significantly greater than that of the cantilever beam with $w=30 \mathrm{~mm}$, which is consistent with the conclusion drawn in section 5.1. Meanwhile, the cantilever beam with $w=30 \mathrm{~mm}$ in Fig. 20(b) is breakdown and the chip separation phenomenon occurs. However, under the same impact conditions, the cantilever beam with width 
$w=10 \mathrm{~mm}$ (as shown in Fig. 20 (a)) did not breakdown, and only obvious plastic bending was observed at the fixed terminal and impact point of the cantilever beam. The reason may be that the cantilever beam with narrow width has smaller mass, smaller inertia, faster response after impact, and will quickly follow the particles move together, so the corresponding threshold value of breakdown velocity is higher. Meanwhile, due to the influence of boundary effect, the fixed terminal of cantilever beam with narrow width has weaker resistance to external load and is more prone to plastic deformation.

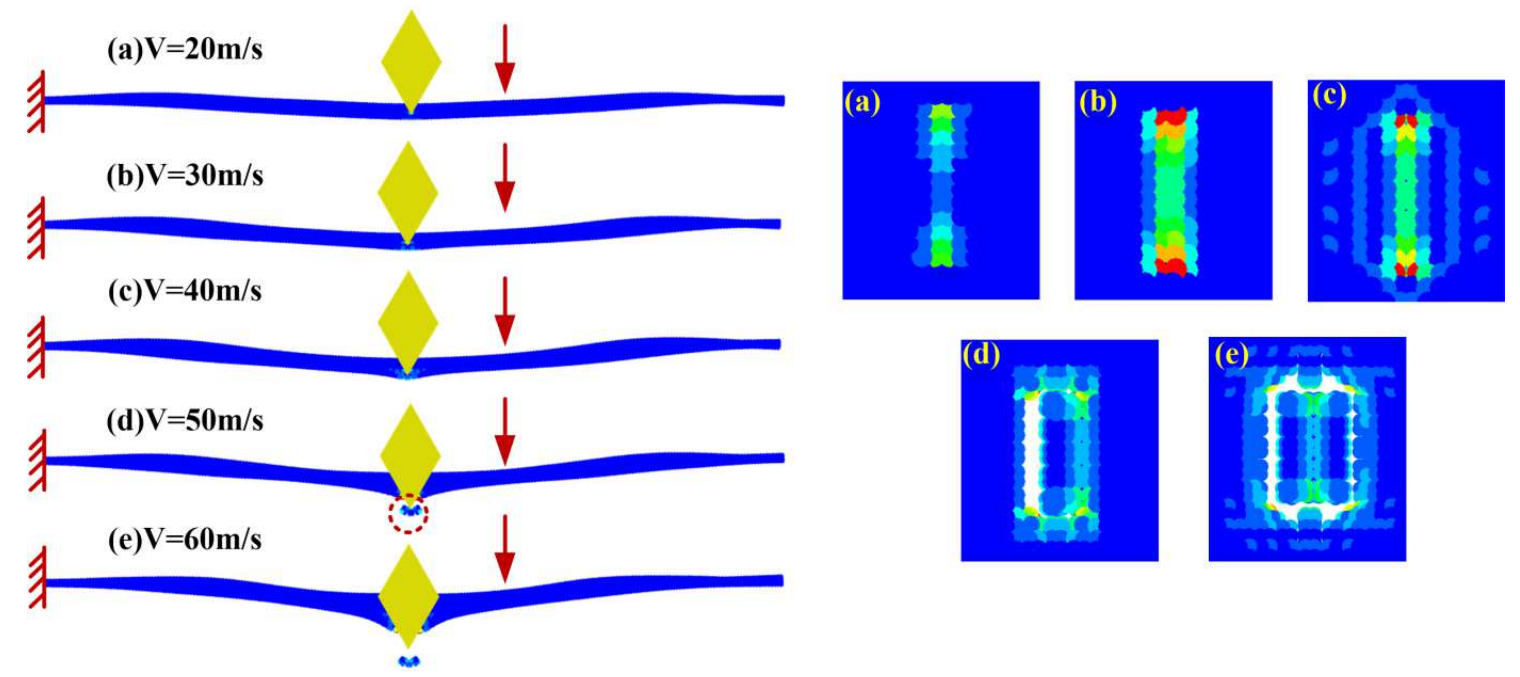

Fig.19. Deformation behavior and crater profiles of cantilever beam at different incident velocities $(l=60 \mathrm{~mm}, w=30 \mathrm{~mm}, h=0.5 \mathrm{~mm})$

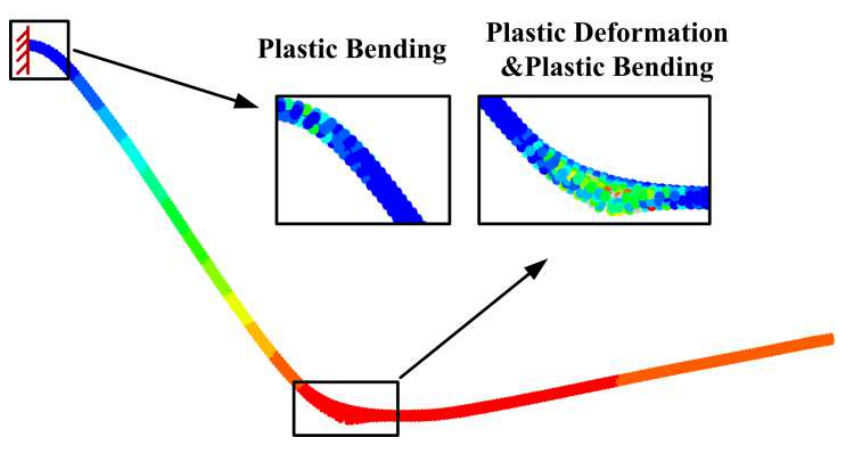

(a) The width of cantilever beam: $w=10 \mathrm{~mm}$

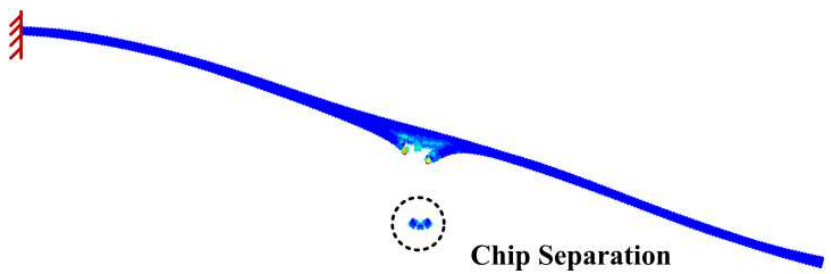

(b) The width of cantilever beam: $w=30 \mathrm{~mm}$

Fig.20. Deformation behavior of cantilever beams with different widths under the same impact condition $\left(\mathrm{V}=60 \mathrm{~m} / \mathrm{s}, \alpha_{i}=90^{\circ}, \theta_{i}=0^{\circ}\right)$

Taking rhomboid-shaped particle impacting the middle position of cantilever beam $(L=60 \mathrm{~mm}, w=30 \mathrm{~mm}$, $h=0.5 \mathrm{~mm}$ ) vertically as an example, Fig. 21 (a) and (b) show the variation of deflection of cantilever beams of different sizes with the incident velocity $V$ of particles. It can be seen that with the increase of the incident 
velocity, the maximum deflection $\gamma$ at the impact position of the cantilever beam and the maximum deflection $\delta$ at the free terminal gradually increase, and the increasing tendency is affected by the width $w$ of the cantilever beam. In section 5-1, the effect of beam width on the maximum deflection of cantilever beam is discussed. Comparing Fig. 21 (a) and (b), the smaller the cantilever beam width $w$, the larger the values of $\gamma$ and $\delta$, which consistent with the conclusion drawn in section 5-1. It must be noticed that when the incident velocity exceeds $40 \mathrm{~m} / \mathrm{s}$ in Fig. 21 (a), the increment of maximum deflection $\delta$ is obviously weakened. The reason may be that after the incident velocity exceeds $40 \mathrm{~m} / \mathrm{s}$, obvious plastic bending occurs at the impact position of the cantilever beam with width of $10 \mathrm{~mm}$, and the free terminal upturned. Therefore, the maximum deflection $\delta$ decreases compared with that when the free terminal does not upturn. In Fig. 21 (b), when the incident velocity exceeds $50 \mathrm{~m} / \mathrm{s}$, the substrate of the cantilever beam is breakdown, and the particles will be embedded in the substrate and move with the cantilever beam together. Therefore, the increment of $\gamma$ and $\delta$ increases rapidly when the velocity more than $50 \mathrm{~m} / \mathrm{s}$.

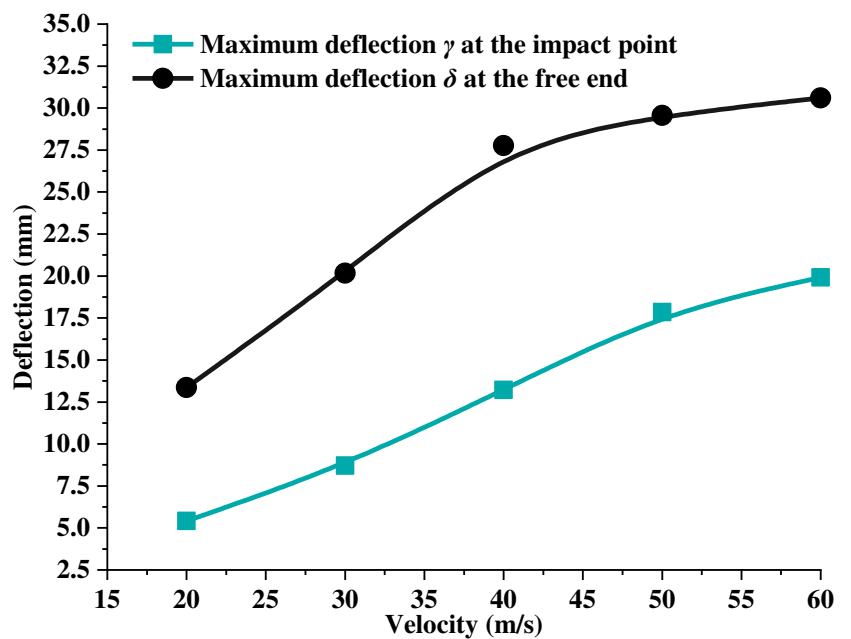

(a) The size of cantilever beam: $L=60 \mathrm{~mm}, \mathrm{w}=10 \mathrm{~mm}, \mathrm{~h}=0.5 \mathrm{~mm}$

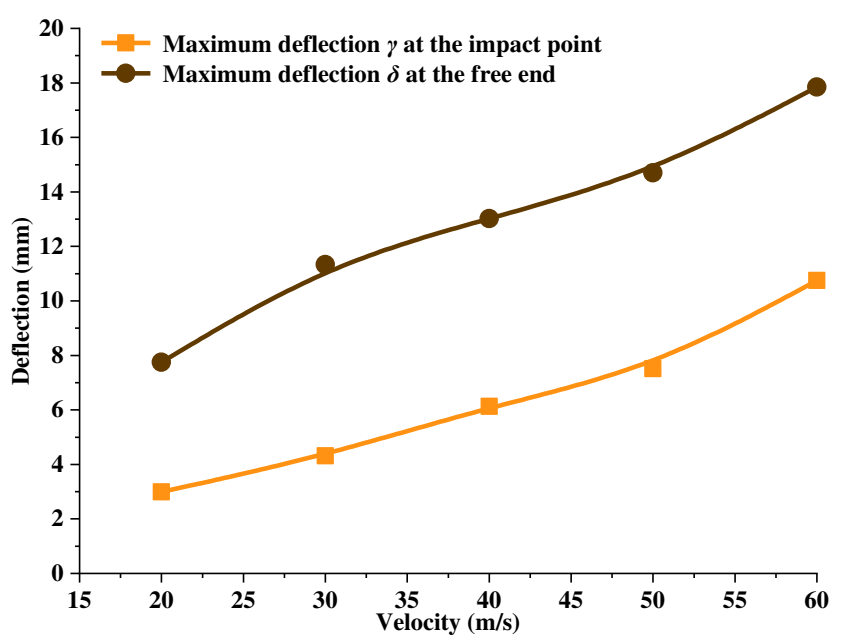

(b) The size of cantilever beam: $L=60 \mathrm{~mm}, \mathrm{w}=30 \mathrm{~mm}, \mathrm{~h}=0.5 \mathrm{~mm}$

Fig.21. Variation of deflection of cantilever beams of different sizes with the incident velocity

\subsection{Effects of impact position}

The impact position of particles is also one of the important factors affecting the maximum deflection of the thin beam. Taking rhomboid-shaped particle impacting the cantilever beam $(L=60 \mathrm{~mm}, w=10 \mathrm{~mm}$, 
$h=0.5 \mathrm{~mm})$ vertically as an example, keep the initial incident conditions of particle unchanged $(\mathrm{v}=20 \mathrm{~m} / \mathrm{s}$, $\alpha_{i}=90^{\circ}, \theta_{i}=0^{\circ}$ ), the positions of $l=15 \mathrm{~mm}, l=30 \mathrm{~mm}$ and $l=45 \mathrm{~mm}$ from the free terminal of the cantilever beam are impacted, respectively, and the obtained crater contour is shown in Fig. 22. Fig. 23 (a) and (b) show the variation of the deflection of cantilever beams of different sizes with the impact position of particles. It can be seen that as the impact position gradually far away from the free terminal of the cantilever beam, the maximum deflection value $\delta$ at the free end gradually decreases. While the maximum deflection value $\gamma$ at the impact position increases first and then decreases. The value of $\gamma$ when the particles impact the middle position of the cantilever beam is higher than that of other positions. This rule is not affected by the width $w$ of the cantilever beam, as shown in Fig. 23 (a) and (b).
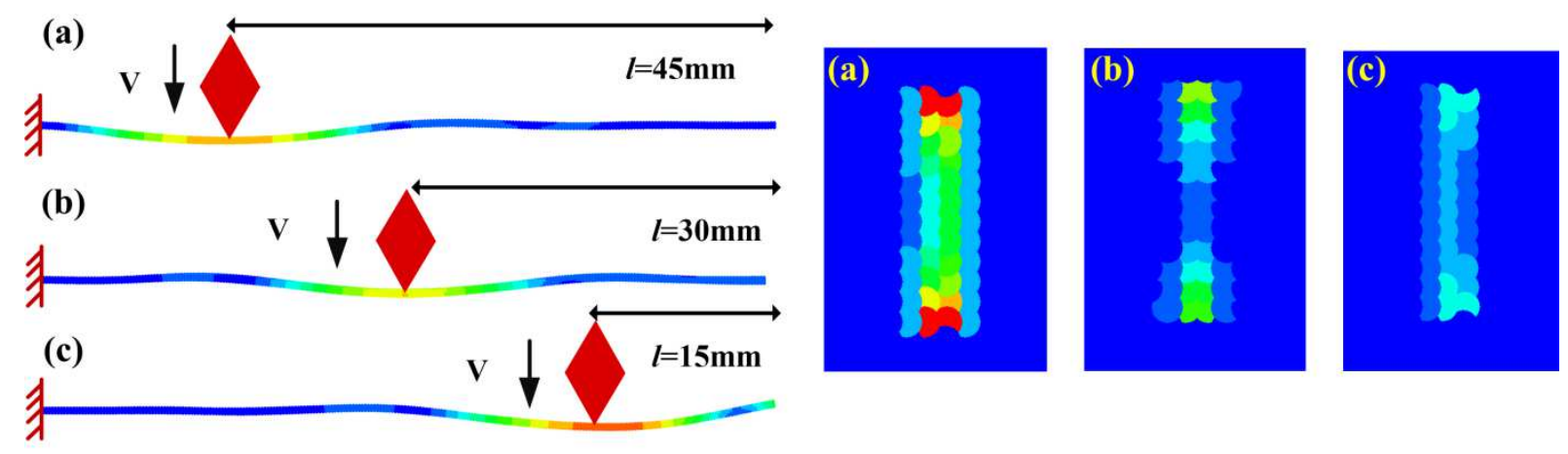

Fig.22. Different impact positions of cantilever beams and corresponding crater contours

Fig.24 shows the energy evolution curves of the entire system when rhomboid-shaped particles impact the cantilever beam $(L=60 \mathrm{~mm}, w=10 \mathrm{~mm}, h=0.5 \mathrm{~mm})$ at different positions. The black line represents the total energy of the system, and the whole process remains constant. The impact process is mainly the transformation of kinetic energy of particles and the internal energy of cantilever beam. The internal energy of cantilever beam includes the energy that causes elastic deformation and plastic deformation. Due to the fact that the cantilever beam exhibits reciprocating vibration after impact, the kinetic energy curve and internal energy curve of the system fluctuate at a certain value. From Fig.24 it can be seen that the steady-state values of the internal energy increment of the system under three impact positions are basically the same, indicating 
that the impact position does not affect the cantilever internal energy increment. However, the proportion of the increment of elastic potential energy and plastic strain energy are not the same. When the impact position is close to the free terminal of the cantilever beam, the elastic deformation of the cantilever beam is larger, and the corresponding elastic potential energy increment is more, and the strain energy used for the plastic deformation of the substrate is less, so the plastic crater contours is small and shallow, as shown in Fig. 22 (c). Similarly, when the impact position is far from the free terminal of the cantilever beam, the increase in elastic potential energy is less. At this time, the strain energy used for the plastic deformation of the substrate is more, so the contour of the plastic crater is larger and deeper, as shown in Fig. 22(a). In addition, it can be seen from Fig. 24 that when the impact position is far away from the free terminal of the cantilever beam, the faster the decrease rate of kinetic energy in the impact process, the faster the increase rate of corresponding internal energy. While when the impact position is close to the free terminal of the cantilever beam, the decrease rate of system kinetic energy and the increase rate of internal energy obviously decrease, which indicates that the response speed of the system close to the fixed terminal is faster than that of far away from the fixed terminal.

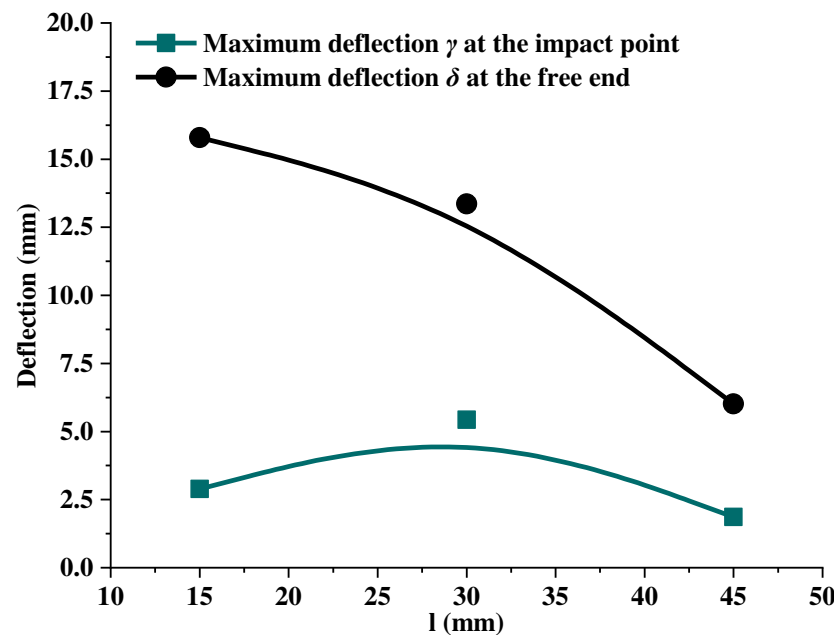

(a) The size of cantilever beam: $L=60 \mathrm{~mm}, \mathrm{w}=10 \mathrm{~mm}, \mathrm{~h}=0.5 \mathrm{~mm}$

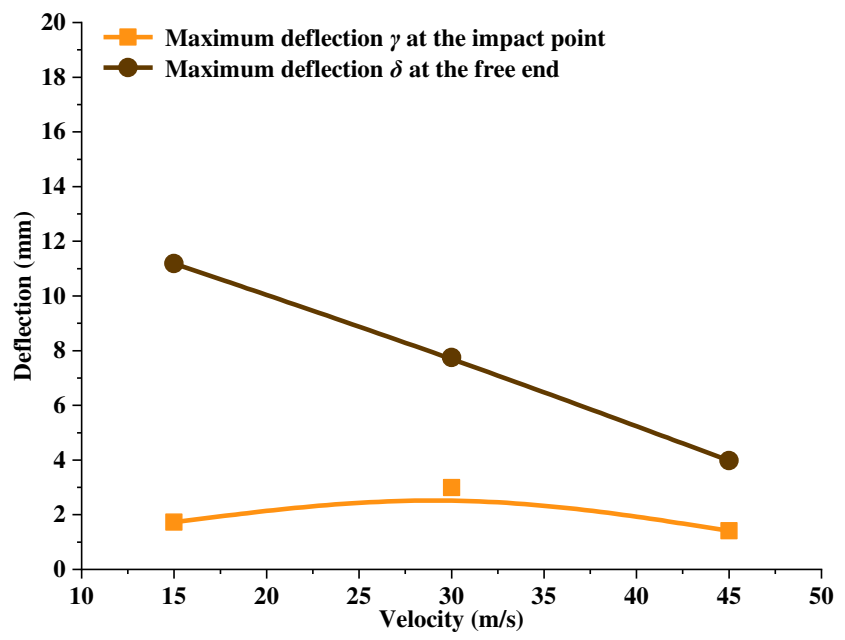

(b) The size of cantilever beam: $L=60 \mathrm{~mm}, \mathrm{w}=30 \mathrm{~mm}, \mathrm{~h}=0.5 \mathrm{~mm}$

Fig.23. Deflection of cantilever beams of different sizes varies with the incident position of particles 


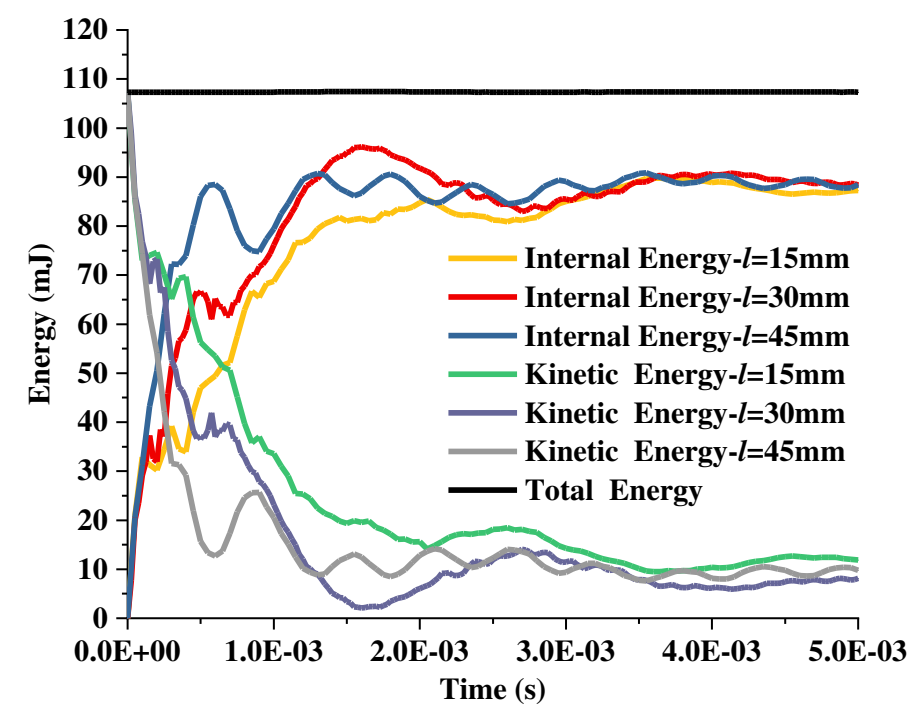

Fig.24. Energy evolution curves of cantilever beam system at different impact positions

\subsection{Effects of impact angle $\alpha_{i}$ and orientation angle $\theta_{i}$}

Relevant researches ${ }^{[19,30]}$ show that the impact angle $\alpha_{i}$ and orientation angle $\theta_{i}$ of the incident particles are also one of the important factors affecting the substrate crater morphology. Rhomboid-shaped particles impact the fixed ductile material substrate orthogonally ${ }^{[31]}$ satisfies the "critical orientation angle criterion", that is, for a given constant impact angle $\alpha_{i}$, there is a critical orientation angle $\theta_{c r i}$, in which the transition from kinetic energy into internal energy is maximized. For initial orientation angles $\theta_{i}<\theta_{c r i}$, the particle rotated forwards after the impact with the target. For values of $\theta_{i}>\theta_{c r i}$, the particle rotated backwards after the impact. For initial orientation angles $\theta_{i}=\theta_{c r i}$, the rotation of particle was greatly suppressed. The critical orientation angle criterion has been proved to be independent of particle shape ${ }^{[32]}$ and properties of substrate material ${ }^{[33]}$. In this section, the effect of impact angle $\alpha_{i}$ and orientation angle $\theta_{i}$ on the process of rhomboid-shaped particles impacting cantilever beam and fixed beam is studied, and the motion behaviors of rhomboid-shaped particle after impact under four different impact angle $\alpha_{i}$ and orientation angle $\theta_{i}$ is given (as shown in Fig.25 and Fig.26) to verify the applicability of the critical orientation angle criterion in impacting cantilever beam and fixe-fixed beam. 


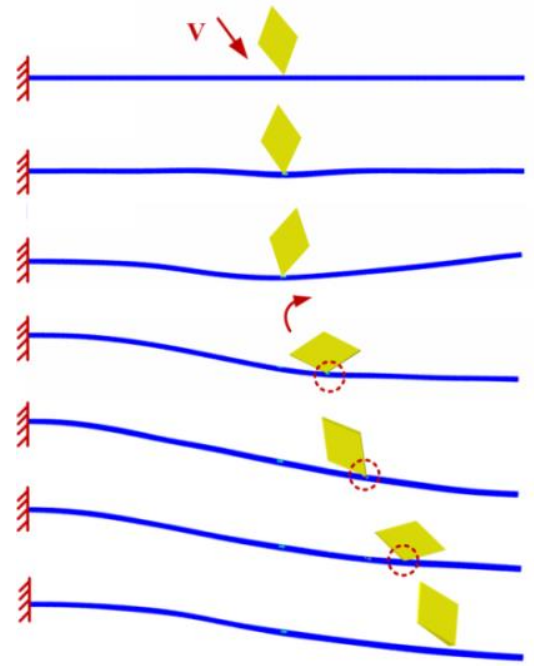

(a) case $1: V=20 \mathrm{~m} / \mathrm{s}, \alpha_{i}=60^{\circ}, \theta_{i}=10^{\circ}$

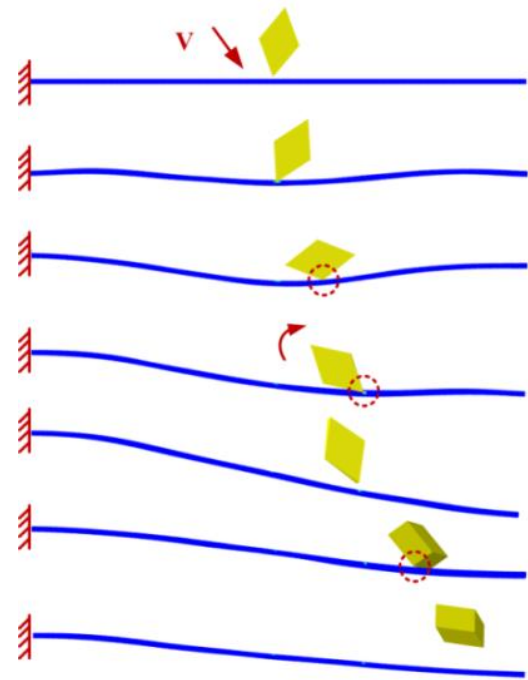

(c) case $3: V=20 \mathrm{~m} / \mathrm{s}, \alpha_{i}=60^{\circ}, \theta_{i}=-10^{\circ}$

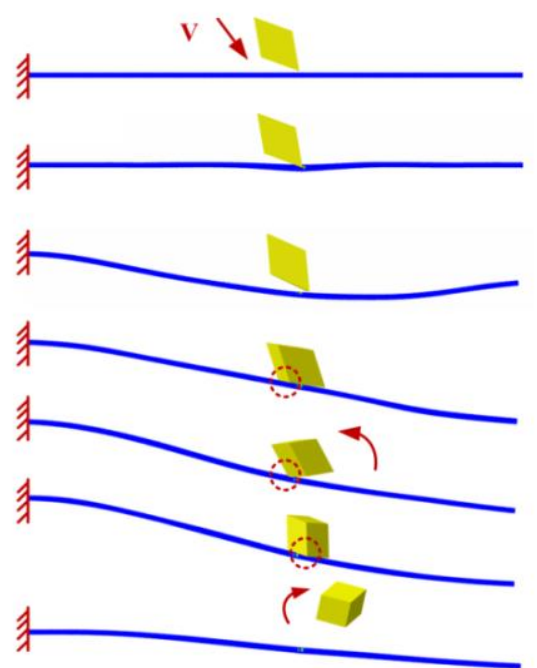

(b) case2: $V=20 \mathrm{~m} / \mathrm{s}, \alpha_{i}=60^{\circ}, \theta_{i}=40^{\circ}$

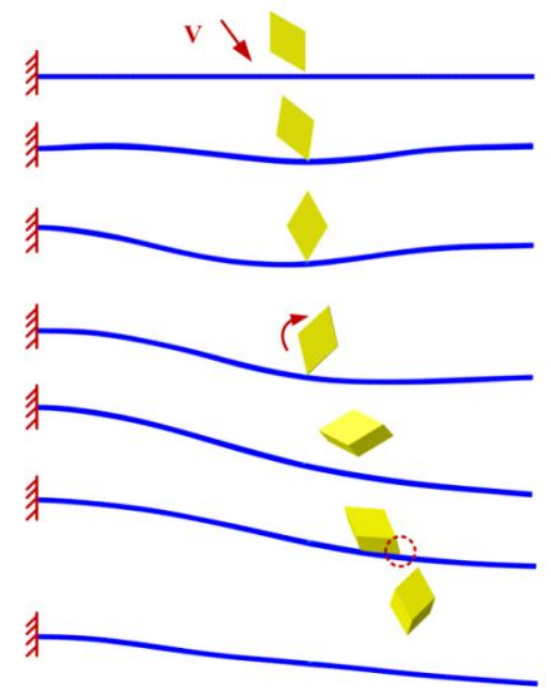

(d) case4: $V=20 \mathrm{~m} / \mathrm{s}, \alpha_{i}=60^{\circ}, \theta_{i}=30^{\circ}$

Fig.25. Influence of impact angle $\alpha_{i}$ and orientation angle $\theta_{i}$ on particle motion behavior when impact cantilever beam

Keep the incident velocity $V=20 \mathrm{~m} / \mathrm{s}$ unchanged, Fig. 25(a)(b)(c)(d) show the motion behaviors of the rhomboid-shaped particles after impacting the Al6061-T6 cantilever beam $(L=60 \mathrm{~mm}, w=10 \mathrm{~mm}, h=0.5 \mathrm{~mm})$, respectively, such as the forward rotation, back rotation, forward rolling and rotation behavior are suppressed, and the deformation characteristics of the cantilever beam. The phenomena are similar with the conclusion obtained from the impact of rhomboid-shaped particles on the ductile fully constrained substrate under the same incident conditions, which proves that the impact of rhomboid-shaped particles on the cantilever beam still satisfies the critical orientation angle criterion. However, it should be noticed that the particles in Fig. 
25(a) have four impacts in the process of forward rotation, which is the same as that in Fig. 25(c), while only one impact occurs on the ductile fully constrained substrate under the same conditions. The reasons may be that: (1) compared with the process of impacting the ductile fully constrained substrate, the direction of normal velocity vector of the rhomboid-shaped particle does not change after the first rebound when impact on the thin cantilever beam. The particle still has a downward movement tendency. As the particles rolling, other tips have the opportunity to contact the substrate again. (2) The initial impact point of the particle is the center of the cantilever beam, the center of the cantilever beam will bend deformation after the first impact, forming a "V" shape. At this time, the actual impact angle $\alpha_{i}$ of particles decreases and the orientation angle $\theta_{i}$ increases, the upturned free terminal of the cantilever beam hinders the subsequent movement of particles. Combining the above factors, the tip of particle has opportunity to contact with the material surface again after the initial rebound, resulting in secondary and third impact. As the impact process continues, the curved cantilever beam begins to rebound, and the free terminal of the cantilever beam contacts with the particles again during the rebound process, resulting in a fourth impact behavior. Meanwhile, part of the elastic potential energy of the cantilever beam is converted into the kinetic energy of the particles, which is higher than before. The reason for the forward rolling behavior in Fig. 25(c), in addition to the above factors, has a lot to do with the degree of inclination at the time of incident. The angle between the incident direction and the long centroid line of particles is too large, which lead to a strong forward rotation of particles after the first impact, and the particle has a tendency to roll forward along the substrate surface, which makes the other tip of the particles contact the material surface again in the process of forward rolling and flying away, resulting in secondary or third impact. In Fig. 25(b), the particle has a backward rotation after impact, which has a "digging" effect on the surface of the material. Due to the lower velocity and the incompletely fixed substrate, the effect of "digging" is weak and no chip separation generates. Meanwhile, the cantilever beam is bent due to the impact of particle, resulting in the decrease of the actual impact angle $\alpha_{i}$ and the increase of orientation 
angle $\theta_{i}$. According to the comprehensive mechanism of the two factors, the other tips of particle has opportunity to contact with the material surface again as particle downward movement, resulting in two or even multiple impacts. Fig. 25(d) shows the critical impact of rhomboid-shaped particle. The rotation behavior of particle is greatly inhibited after impacting, while still has a weak forward rotation movement trend. The reasons of secondary impact are similar with that in Fig. 25 (a) and (c).

Keep the incident velocity $V=20 \mathrm{~m} / \mathrm{s}$ unchanged, Fig. 26(a)(b)(c)(d) show the motion behaviors of the rhomboid-shaped particles after impacting the Al6061-T6 fixed-fixed beam ( $L=60 \mathrm{~mm}, w=10 \mathrm{~mm}, h=0.5 \mathrm{~mm})$, respectively, such as the forward rotation, back rotation, forward rolling and rotation behavior are suppressed, and the deformation characteristics of the cantilever beam. The initial incident conditions of the four cases are consistent with those in Fig. 25. It is observed that the process of rhomboid-shaped particles impacting the fixed-fixed beam still satisfies the critical orientation angle criterion, but the impact times are significantly reduced compared with that of the cantilever beam under the same impact conditions. For example, only two impacts are generated in Fig. 26(a), which is two times less than that in Fig. 25(a). The reason may be that the direction of the normal velocity vector has changed after the particle impacting the fixed-fixed beam for the first time, so the tendency of particle to roll downward disappears. Meanwhile, the fixed-fixed beam shows reciprocating vibration after impact, which makes the tip of particle has opportunity to contact the fixed-fixed beam again, resulting in secondary impact. However, under the same conditions, only one impact occurs when the ductile fully constrained substrate is impacted, which is related to the fact that substrate does not produce reciprocating vibration. The reason of the three impacts in Fig. 26 (c) are similar to that in 25 (c) and will not be described here. The particle shows backward rotation in Fig. 26 (b), which has a "digging" effect on the substrate surface. Due to the lower velocity and the incompletely fixed substrate, the effect of "digging" is weak and no chip separation happens. Meanwhile, the fixed-fixed beam is bent due to the impact of particles, and after reaching maximum deflection $\gamma^{\prime}$, the fixed-fixed beam starts to rebound, which generate a certain 
reverse pushing effect on the particle tip. The particles rotate anticlockwise and leave the substrate surface with a large rebound angle $\alpha_{r}$, therefore, only one impact occurs in the whole impact process. Fig. 26(d) shows the critical impact case. After the impact, the rotation behavior of the particles is greatly suppressed, but there is still a slight tendency of forward rotation. Due to the change of the normal velocity vector direction during the impact process, only one impact occurs, which is similar to the process of impacting the fully constrained substrate.

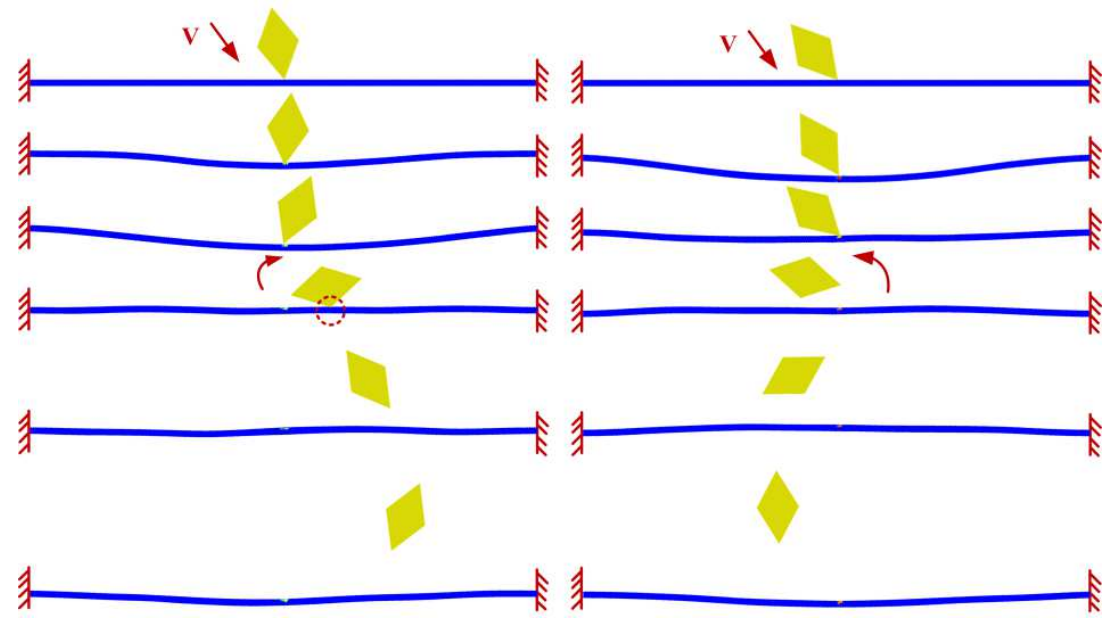

(a) case1: $V=20 \mathrm{~m} / \mathrm{s} \alpha_{i}=60^{\circ} \theta_{i}=10^{\circ}$

(b) case $2: V=20 \mathrm{~m} / \mathrm{s} \alpha_{i}=60^{\circ} \theta_{i}=40^{\circ}$

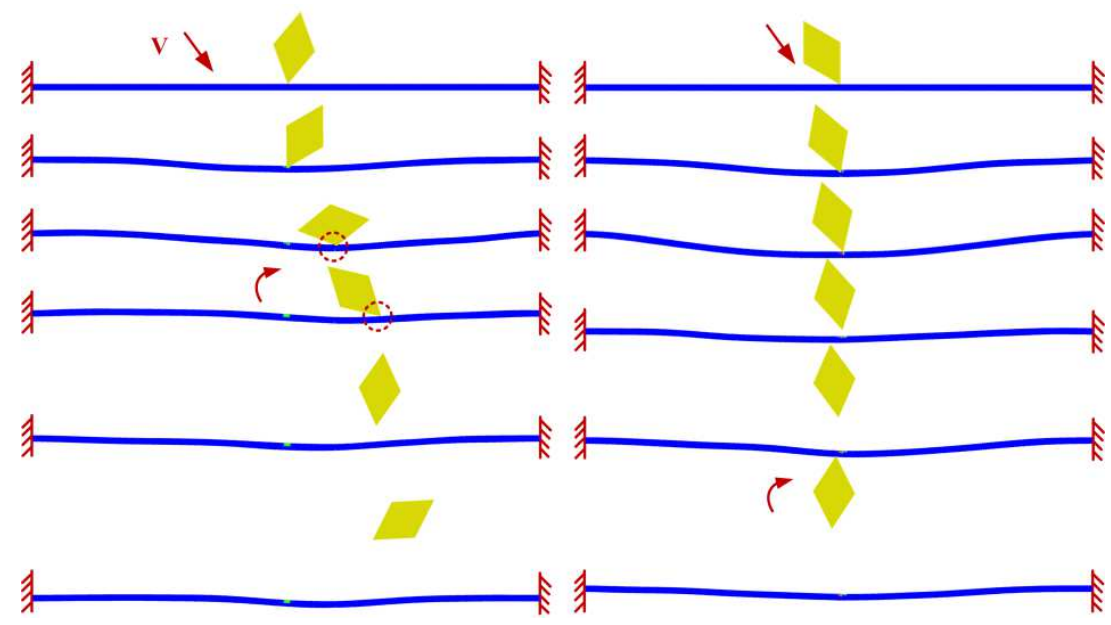

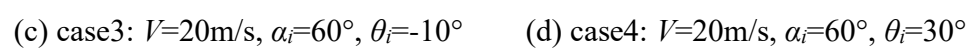

Fig.26. Influence of impact angle $\alpha_{i}$ and orientation angle $\theta_{i}$ on particle motion behavior when impact fixed-fixed beam 


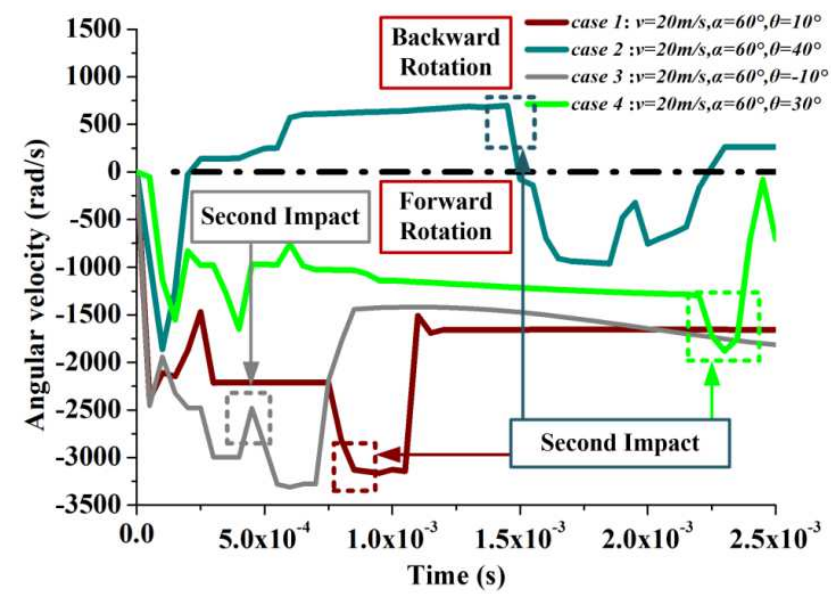

(a) Rhomboid-shaped particle impact cantilever beam

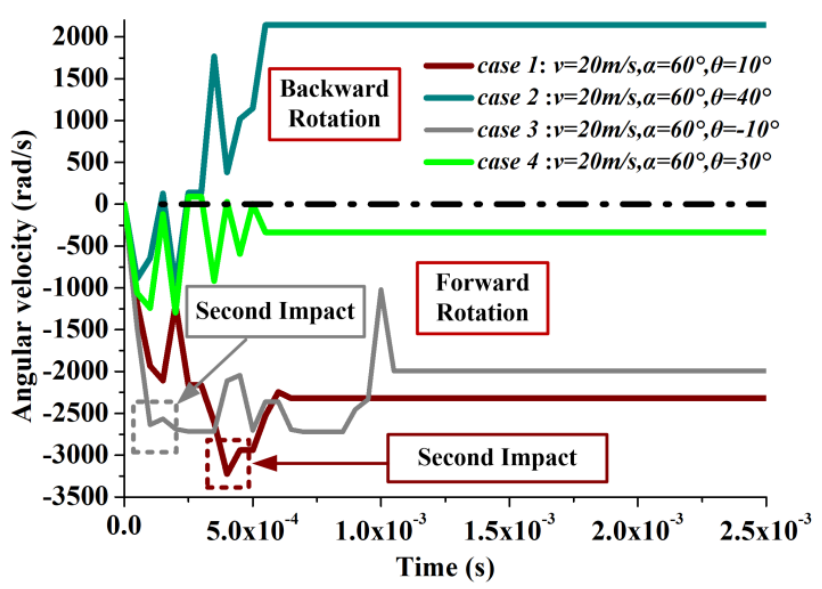

(b) Rhomboid-shaped particle impact fixed-fixed beam

Fig.27. History curves of particle angular velocity with time under four impact conditions

From the above analysis, it can be seen that there is a high probability of multiple impacts occurred when rhomboid-shaped particles impacting cantilever beam under low-speed conditions, which has a lot to do with the direction of the particle normal velocity vector after the initial impact rebound. At the same time, the rotation behavior of particles after impact still satisfies the critical orientation angle criterion. The combination of different impact angle $\alpha_{i}$ and orientation angle $\theta_{i}$ determines the particle rotation behavior. Fig. 27 (a) shows the history curves of angular velocity of particles after impacting the cantilever beam with time under four impact cases. It can be seen from the curves that at the end of the first impact, the particles have a tendency of forward rotation, and then the particles in case 2 change into backward rotation (the angular velocity value is positive), while the particles in cases 1, 3 and 4 always keep moving forward. It is remarkable that the secondary impact time of case 4 is significantly later than that of the other three conditions when the secondary impact occurs, which indicates that the rotation behavior of particle under the critical impact case is significantly suppressed. The secondary impact phenomenon occurs first in case 3 due to the fact that the initial orientation angle is negative. After the secondary impact, the subsequent motion behavior of particles becomes unpredictable by reason of the obvious changes of contact conditions and particle motion parameters, for instance, the particles in case 2 change into forward rotation. In Fig. 27(b), except for cases 2 and 4 
without secondary impact, the time of secondary impact in the cases 1 and 3 is earlier than that in the impact cantilever beam. The reasons may be that due to the small elastic deformation range of the fixed-fixed beam and the rapid rebound after the impact position reaches the maximum deflection $\gamma^{\prime}$, therefore the convex part of the beam has the opportunity to contact the particle again after rebound, resulting in a secondary impact effect.
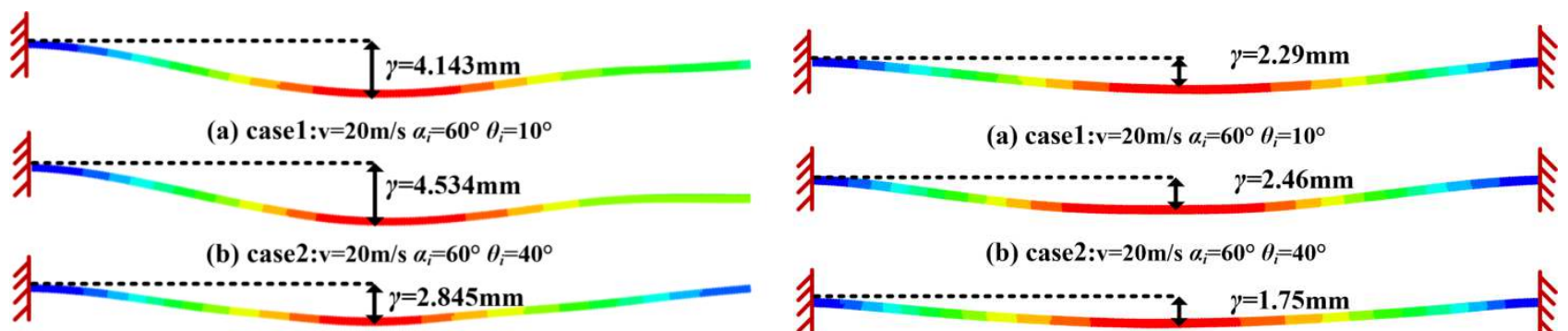

(c) case $3: \mathrm{v}=20 \mathrm{~m} / \mathrm{s} \alpha_{i}=60^{\circ} \theta_{i}=-10^{\circ}$

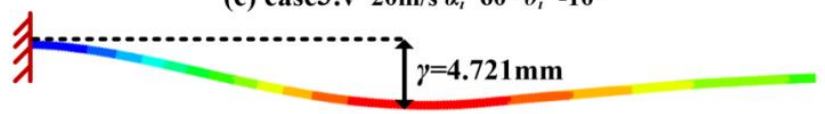

(d) case $4: \mathrm{v}=20 \mathrm{~m} / \mathrm{s} \alpha_{i}=60^{\circ} \theta_{i}=30^{\circ}$

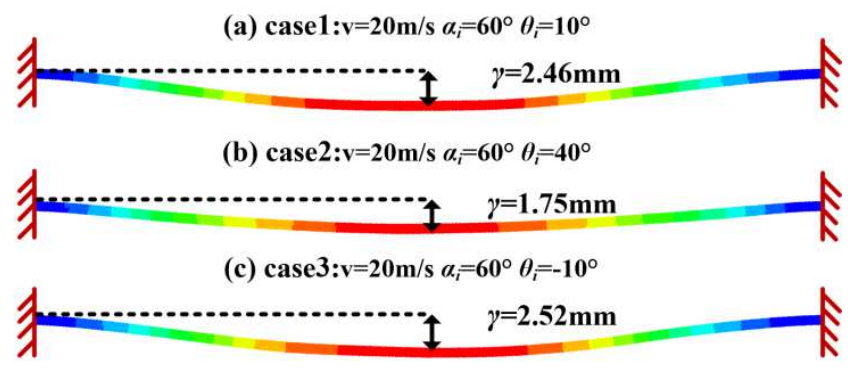

(d) case4:v $=20 \mathrm{~m} / \mathrm{s} \quad \alpha_{i}=60^{\circ} \theta_{i}=30^{\circ}$

(a) Rhomboid-shaped particle impact cantilever beam

(b) Rhomboid-shaped particle impact fixed-fixed beam

Fig.28. Maximum deflection $\gamma$ at the impact point caused by rhomboid-shaped particle under four impact conditions

Figure 28(a) shows the comparison of the maximum deflection $\gamma$ at the impact point of the cantilever beam after the initial impact of rhomboid-shaped particles under four impact conditions. It can be seen that under the critical impact condition (case4), the cantilever beam has the largest bending extent after the initial impact of the particle, which is $4.721 \mathrm{~mm}$. Meanwhile, the transition from kinetic energy of particle into internal energy (elastic potential energy and the plastic strain energy) is maximized, which is consistent with the energy evolution law of the rhomboid-shaped particles impacting the fully constrained substrate. In case 3 , the maximum deflection $\gamma$ at the impact point is the minimized after the initial impact of the particle, which has a lot to do with the inclination extent of the particle when incident. The larger angle between the incident direction and the long centroid of the particle results in a major forward rotation velocity after the first impact of the particle, the kinetic energy loss of the particle is smaller, so as the deformation extent of the cantilever 
beam. The variation tendency of maximum deflection $\gamma^{\prime}$ at the impact position of fixed-fixed beam in Fig. 28(b) is basically the same as that of cantilever beam. The maximum bending deformation occurs under the critical impact condition (case4) and the minimum deformation also occurs in forward rolling impact condition (case3). The reason for the above phenomena is basically the same as that of occurred on cantilever beam.

\subsection{Effects of substrate thickness}

The thickness $h$ of the substrate is one of the important factors affecting the vibration characteristics of the fixed-fixed beam after being impacted by particles. Fig. 29 shows the variation of amplitude at impact position with time under the same impact condition $\left(\mathrm{V}=34.75 \mathrm{~m} / \mathrm{s}, \alpha_{i}=90^{\circ}, \theta_{i}=31^{\circ}\right)$ for fixed-fixed beams with different thicknesses. It can be seen that the impact point of the fixed-fixed beam will undergo reciprocating vibration with reduced amplitude after the impact. As the thickness of the beam increases, the maximum deflection value $\gamma^{\prime}$ at the impact position gradually decreases, the amplitude tends to zero more quickly in the same time, and the vibration frequency increases significantly. Therefore, it is reasonable to believe that with the increase of the thickness of the fixed beam, the macroscopic vibration behavior of the beam will gradually disappear. In addition, it should be noticed that the fixed-fixed beams with different thicknesses fluctuate at different values. In Fig. 29 (a) (b) (c) (d), the four fixed-fixed beams with different thicknesses fluctuates at four values of $-1 \mathrm{~mm},-0.63 \mathrm{~mm},-0.13 \mathrm{~mm}$ and $0 \mathrm{~mm}$, respectively. The reason may be that the smaller the thickness of the beam, the greater the extent of deformation under the same impact condition, and the location of impact point and fixed terminal are more likely to generate plastic bending, resulting in the fluctuates of the impact point moving down compared with the initial position. With the increase of beam thickness, the extent of plastic bending decreases and the beam tends to fluctuate around the initial position. 


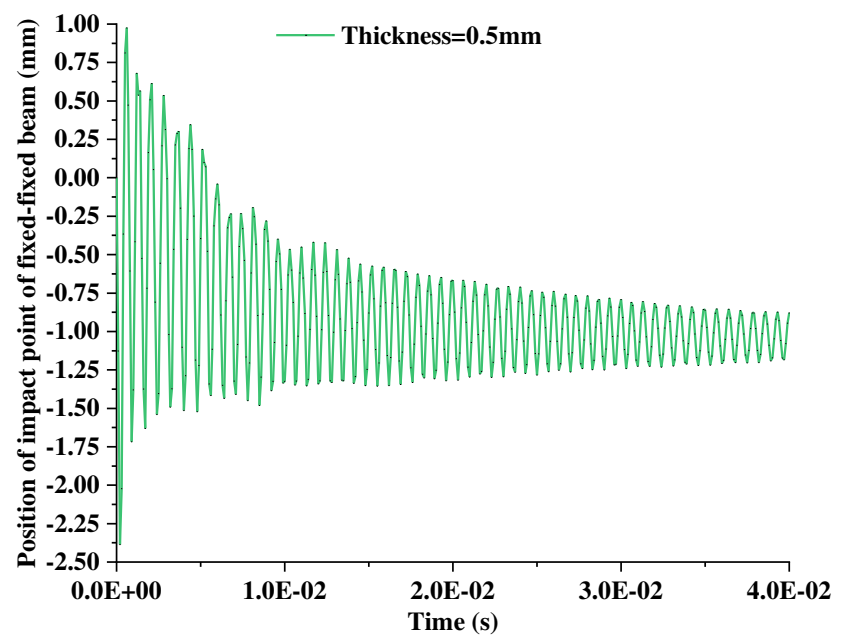

(a) Fixed-fixed beam: thickness $\mathrm{h}=0.5 \mathrm{~mm}$

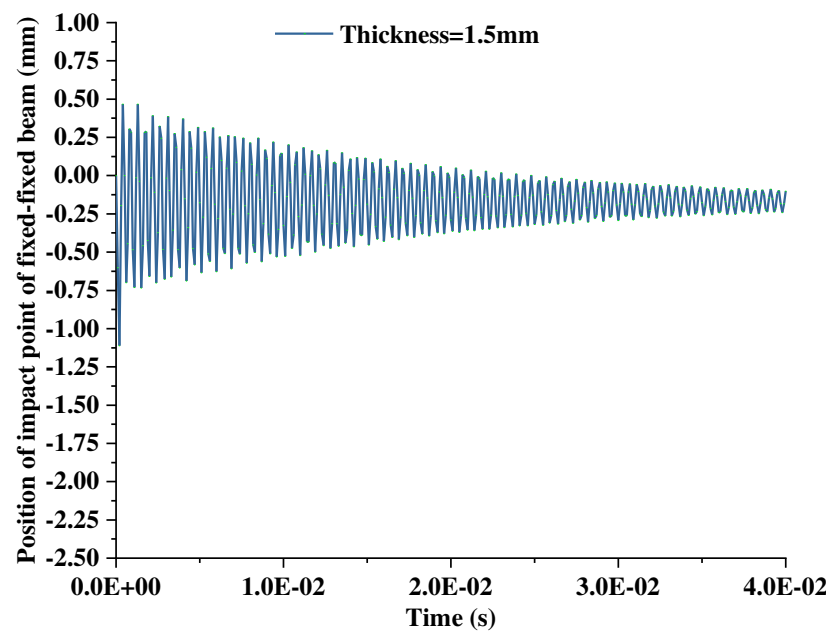

(c) Fixed-fixed beam: thickness $\mathrm{h}=1.5 \mathrm{~mm}$

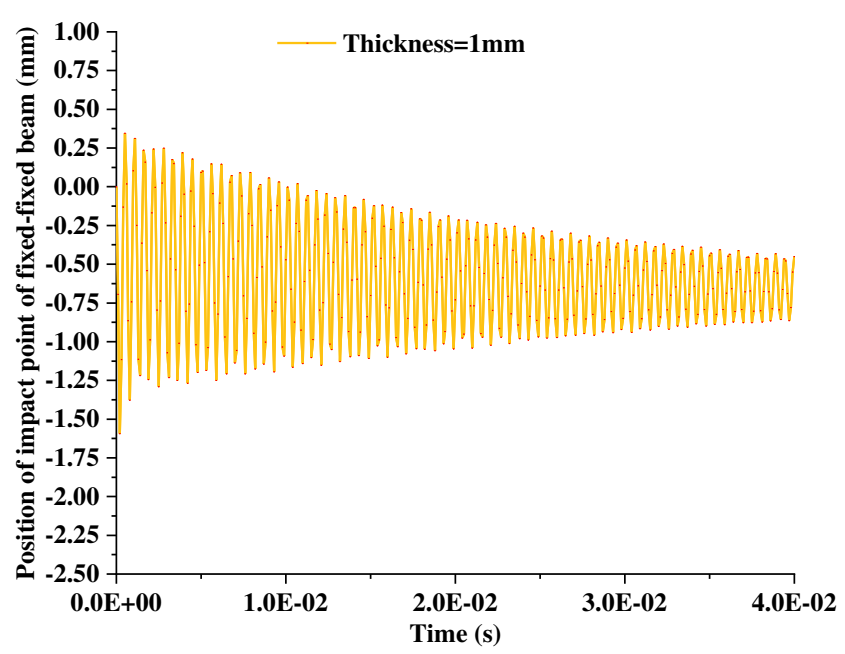

(b) Fixed-fixed beam: thickness $\mathrm{h}=1 \mathrm{~mm}$

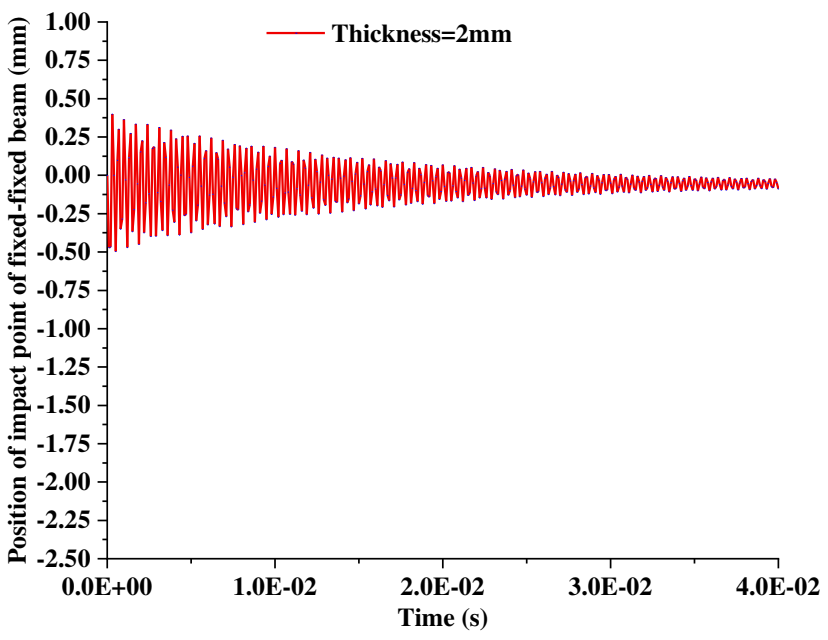

(d) Fixed-fixed beam: thickness $\mathrm{h}=2 \mathrm{~mm}$

Fig.29. Variation of amplitude with time of fixed-fixed beams with different thickness after impact

\section{Conclusions}

In this paper, the erosion mechanism and deformation characteristics of the metal thin plate impacted by rhomboid-shaped particles were studied systematically by means of experiment and numerical simulation. A pneumatic ejection and imaging system for solid particles was built, and physical experiments were carried out for rhomboid-shaped particles impacting A16061-T6 cantilever beams and fixed-fixed beams. The motion behavior of particles and deformation characteristics of beam substrate (such as elastic deformation, plastic bending and crater forming) were captured by high-speed imaging system. Meanwhile, the numerical models 
of rhomboid-shaped particles impacting cantilever beam and fixed-fixed beam were established based on the FEM-SPH coupled method, and the accuracy of the model was verified by comparing the simulated predicted results with experimental measured results. Subsequently, the effects of the geometrical parameters of the beam substrate, the incident conditions of the particle and the initial impact position on the elastic-plastic deformation of the beam substrate and the rebound behavior of the particles were further analyzed by the FEM-SPH coupled numerical model. The results obtained are as follows:

(a) Taking rhomboid-shaped particle impacting the middle position of cantilever beam vertically as an example, the smaller the width $w$ of the beam substrate, the greater the extent of elastic deformation and plastic bending after particle impacting, and the smaller the extent of corresponding plastic crater forming (such as the depth of the crater). The maximum deflection $\gamma$ at the impact position and the maximum deflection $\delta$ at the free terminal of the cantilever beam decrease with the increase of the beam base width $w$.

(b) When the particle incident velocity $V$ increases to a certain threshold $v^{\prime}$, the beam substrate will be breakdown, and the threshold $v^{\prime}$ is related to the dimension parameters of the beam substrate. Taking rhomboid-shaped particle impacting the middle position of cantilever beam vertically as an example, the larger the beam width $w$, the smaller the corresponding threshold value of breakdown velocity $v^{\prime}$.

(c) The farther the initial impact position of particle is from the free terminal of the cantilever, the smaller the maximum deflection value $\delta$ at the corresponding free terminal. The growth rate of the internal energy in the model system increases with the increase of $l$, while the increment of the internal energy of the system is basically independent of the initial impact position.

(d) The initial incident angle $\alpha_{i}$ and orientation angle $\theta_{i}$ determine the motion behavior and rebound law of particles after impacting the beam substrate. The impact of rhomboid-shaped particles on the cantilever beam and fixed-fixed beam still satisfies the critical orientation angle criterion, and the deflection value at impact position of beam substrate is the maximized under the critical impact condition. Under the same 
incident conditions, the impact times of particles on cantilever beam are more than those on the fixed-fixed beam.

(e) The thickness $h$ of the substrate is an important factor affecting the vibration characteristics of the beam. Taking rhomboid-shaped particle impacting the middle position of fixed-fixed beam as an example, with the increase of beam substrate thickness $h$, the maximum deflection value $\gamma^{\prime}$ at the impact position gradually decreases and the vibration frequency increases significantly. The fixed-fixed beams with different thicknesses fluctuate at different values, which is caused by plastic deformation and plastic bending in the impact position and fixed terminal of the thin substrate.

\section{Credit author statement}

Mingchao Du: Data curation, validation, and complete the first draft of the paper. Zengliang Li: Supervision. Xiangwei Dong: Put forward the idea of analysis method. Chunyong Fan: Visualization, Investigation. Jiaqi Che: Simulation. Yanwen Zhang: Experiment.

\section{Acknowledgments}

The authors would like to thank 'National Major Science and Technology Projects (2016ZX05011004-002), Natural Science Foundation of Shandong Province (CN) (ZR2018MA028) and Postgraduate Innovation Engineering (YCX2020065)' for the financial support.

\section{Reference}

[1] Shankar M R, Chandrasekar S, Compton W D, et al. Characteristics of aluminum 6061-T6 deformed to large plastic strains by machining. Materials ence \& Engineering A, 2005, 410(Nov):364-368.

[2] Mahabunphachai S, Koc M. Investigations on forming of aluminum 5052 and 6061 sheet alloys at warm temperatures. Materials \& Design, 2010, 31(5):2422-2434.

[3] Djavanroodi F, Derogar A. Experimental and numerical evaluation of forming limit diagram for Ti6Al4V titanium and Al6061-T6 aluminum alloys sheets. Materials \& Design, 2010, 31(10):4866-4875. 
[4] Salimianrizi A, Foroozmehr E, Badrossamay M, et al. Effect of Laser Shock Peening on surface properties and residual stress of Al6061-T6. Optics and Lasers in Engineering, 2016, 77(FEB.):112-117.

[5] Ghahremaninezhad A, Ravi-Chandar K. Ductile failure behavior of polycrystalline Al 6061-T6. International Journal of Fracture, 2012, 174(2):177-202.

[6] Manes A, Pagani M, Saponara M, et al. Metallographic characterisation of Al6061-T6 aluminium plates subjected to ballistic impact. Materials ence \& Engineering A, 2014, 608:207-220.

[7] Takaffoli M, Papini M. Numerical simulation of solid particle impacts on A16061-T6 part I: Three-dimensional representation of angular particles. Wear, 2012, 292-293(none):100-110.

[8] Takaffoli M, Papini M. Numerical simulation of solid particle impacts on Al6061-T6 Part II: Materials removal mechanisms for impact of multiple angular particles. Wear, 2012, 296(1-2):648-655.

[9] Takaffoli M, Papini M. Material deformation and removal due to single particle impacts on ductile materials using smoothed particle hydrodynamics. Wear, 2012, 274-275(none):50-59.

[10] Hadavi V, Papini M. Numerical modeling of particle embedment during solid particle erosion of ductile materials. Wear, 2015: 310-321.

[11] Salman, A. D., Biggs, C. A., Fu, J., Angyal, I., Szabo, M., \& Hounslow, M. J. An experimental investigation of particle fragmentation using single particle impact studies. Powder Technology, 2002, 128(1):36-46.

[12] Macdonald B J. A computational and experimental analysis of high energy impact to sheet metal aircraft structures. Journal of Materials Processing Tech, 2002, 124(1-2):92-98.

[13] Mccarthy M A, Xiao J R, Mccarthy C T, et al. Modelling Bird Impacts on an Aircraft Wing - Part 2: Modelling the Impact with an SPH Bird Model. International Journal of Crashworthiness, 2005, 10(1): 51-59.

[14] Chen W, Hudspeth M, Guo Z, et al. Multi-scale experiments on soft body armors under projectile normal impact. International Journal of Impact Engineering, 2017: 63-72.

[15] Silnikov M V, Guk I V, Nechunaev A F, et al. Numerical simulation of hypervelocity impact problem for spacecraft 
shielding elements. Acta Astronautica, 2017: 56-62.

[16] Gart S, Mates J E, Megaridis C M, et al. Droplet Impacting a Cantilever: A Leaf-Raindrop System. Physical Review Applied, 2015, 3(4):044019.

[17] Li Rong W, Guang Jun Y, Yang Y, et al. Study on Vibration Characteristics of Single Granular Rock Direct Impact Metal Plate. Coal Technology, 2017, 36(11): 213-216.

[18] Jin gui WANG. Principle and technology of gas gun. Beijing: National Defense Industry Press, 2001.

[19] Takaffoli M, Papini M. Finite element analysis of single impacts of angular particles on ductile targets. Wear, 2009, 267(1):144-151.

[20] Liu, G.R., Liu, M. B.: Smoothed particle hydrodynamics: a mesh-free particle method. World Scientific, Singapore (2004).

[21] Dong XW, Liu GR, Li Z, et al. A smoothed particle hydrodynamics (SPH) model for simulating surface erosion by impacts of foreign particles. Tribology International, 2016, 95:267-278.

[22] Dong X, Li Z, Feng L, et al. Modeling, simulation, and analysis of the impact(s) of single angular-type particles on ductile surfaces using smoothed particle hydrodynamics. Powder Technology, 2017, 318:363-82.

[23] Hu D, Han X, Xiao Y, et al. Research developments of smoothed particle hydrodynamics method and its coupling with finite element method. Chinese Journal of Theoretical \& Applied Mechanics, 2013, 45(5): 639-652.

[24] Johnson GR. Linking of Lagrangian particle methods to standard finite element methods for high velocity impact simulations. Nuclear Engineering and Design, 1994, 150(2-3): 265-274.

[25] Zhang Z, Qiang H, Gao W. Coupling of smoothed particle hydrodynamics and finite element method for impact dynamics simulation. Engineering Structures, 2011, 33(1): 255-264.

[26] Johnson G R, Cook W H. A constitutive model and data for metals subjected to large strains, high strain rates and high temperatures. Proceedings of the 7th International Symposium on Ballistics. 1983, 21: 541-547.

[27] Johnson G R, Cook W H. Fracture characteristics of three metals subjected to various strains, strain rates, 
temperatures and pressure. Engineering Fracture Mechanics, 1985, 21(1):31-48.

[28] Randles PW, Libersky LD. Smoothed particle hydrodynamics: some recent improvement and applications. Comput. Methods Appl. Mech. Eng. 1996, 139(1-4):375-408.

[29] Kim H K, Park E T, Song W J, et al. Experimental and numerical investigation of the high-velocity impact resistance of fiber metal laminates and Al 6061-T6 by using electromagnetic launcher. Journal of Mechanical Science \& Technology, 2019, 33(3):1219-1229.

[30] Takaffoli M, Papini M. Material deformation and removal due to single particle impacts on ductile materials using smoothed particle hydrodynamics. Wear, 2012, 274-275(none):50-59.

[31] Azimian M, Schmitt P, Bart H J. Numerical investigation of single and multi-impacts of angular particles on ductile surfaces. Wear, 2015, 342-343:252-261.

[32] Du M, Li Z, Dong X, et al. Single Pyramid-Shaped Particle Impact on Metallic Surfaces: A 3D Numerical Simulation and Experiment. Tribology Letters, 2019, 67(4) -108: 2-15.

[33] Du M, Li Z, Dong X, et al. Experiment and simulation study of erosion mechanism in float glass due to rhomboid particle impacts. International Journal of Impact Engineering, 139. 


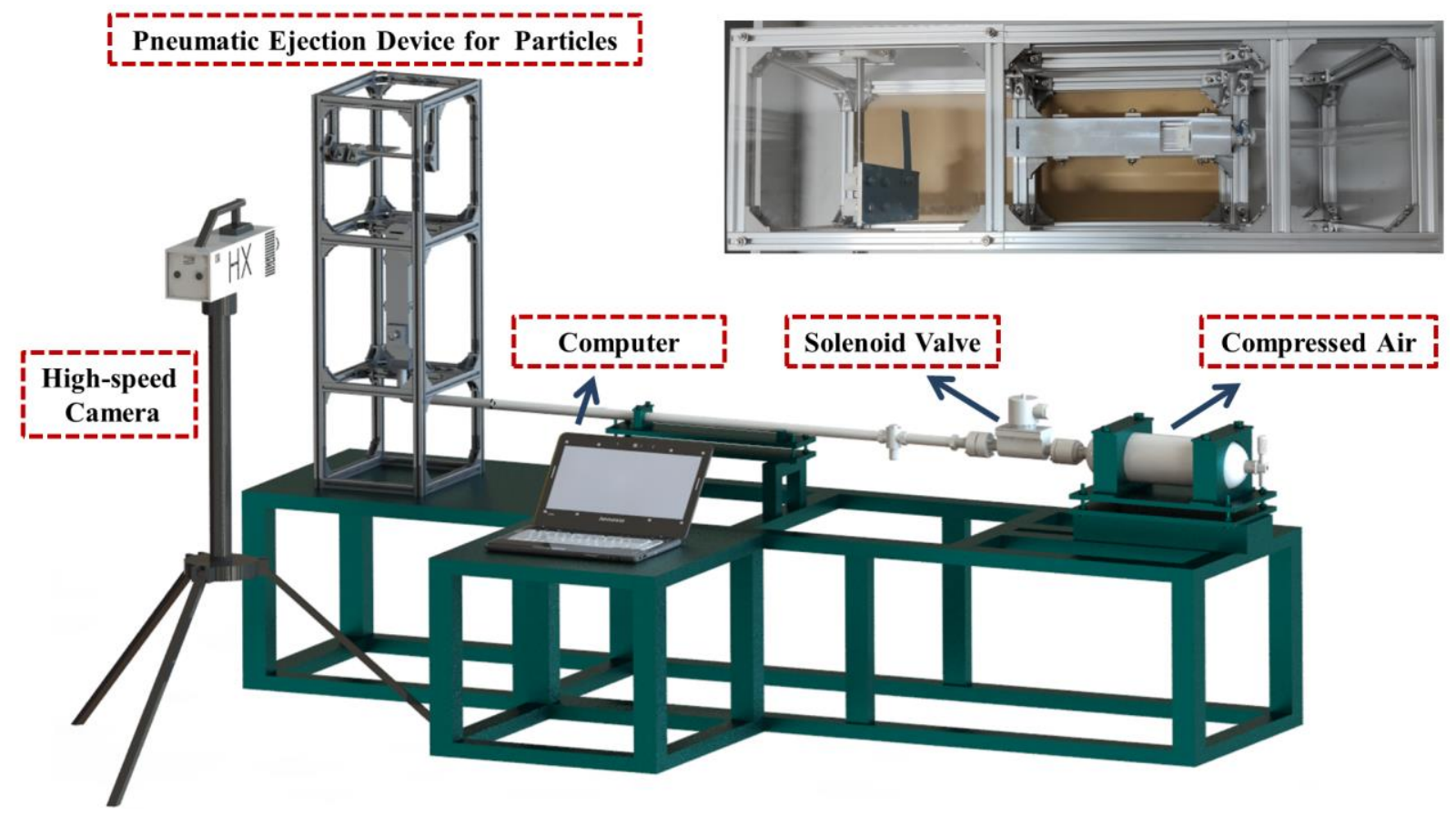

Fig.1. Pneumatic Ejection and Imaging System for Solid Particles 


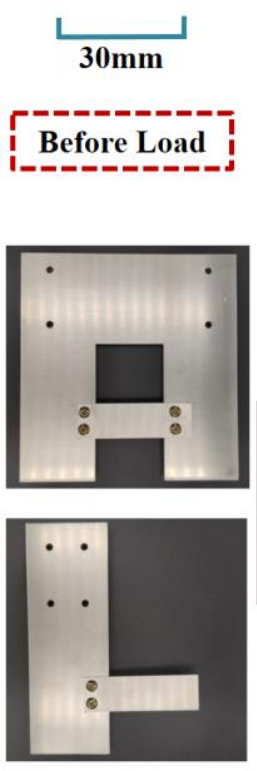

Target Holder
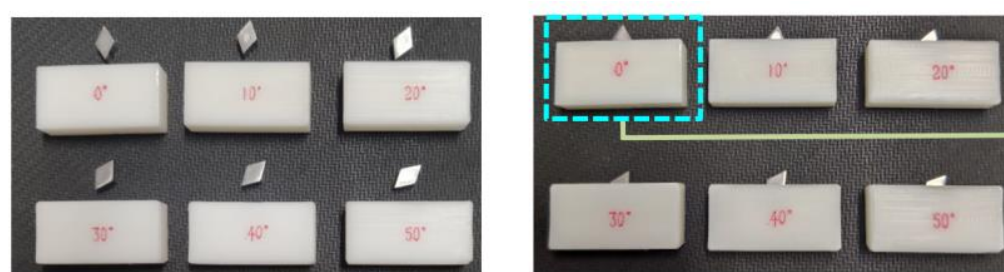

Particle Holder and Adjustment of Orientation Angle $\theta_{i}$

$$
\text { Particle Holder and Adjustment of Orientation Angle } \theta_{i}
$$
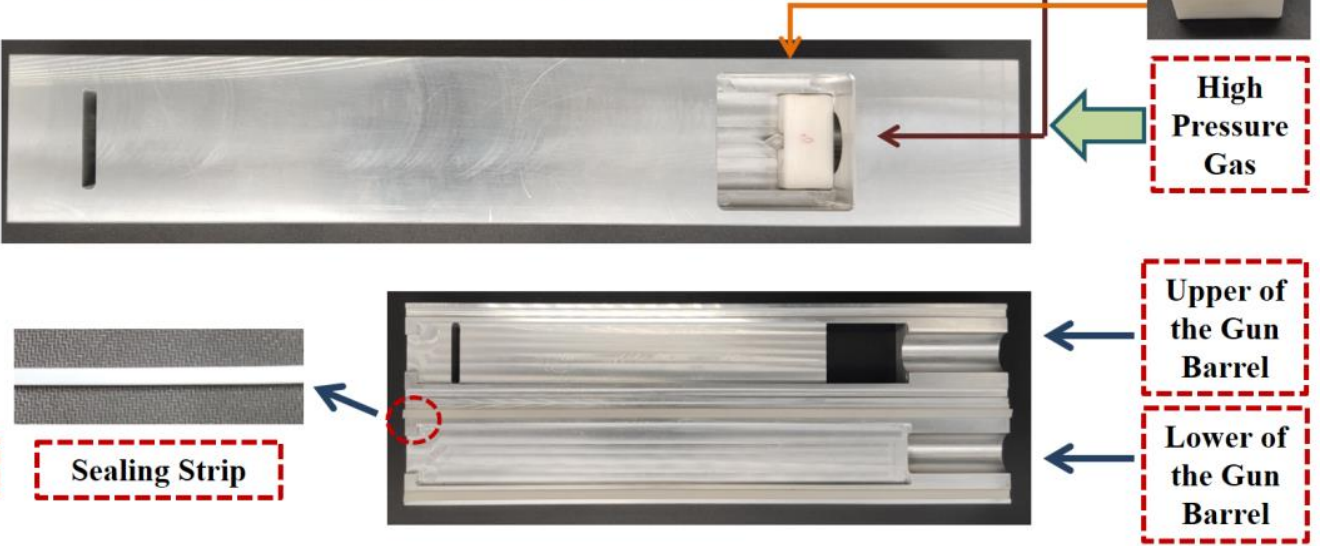

Fig.2. Adjustment of particle orientation angle $\theta_{i}$ and exhibition of variable cross-section gun barrel 


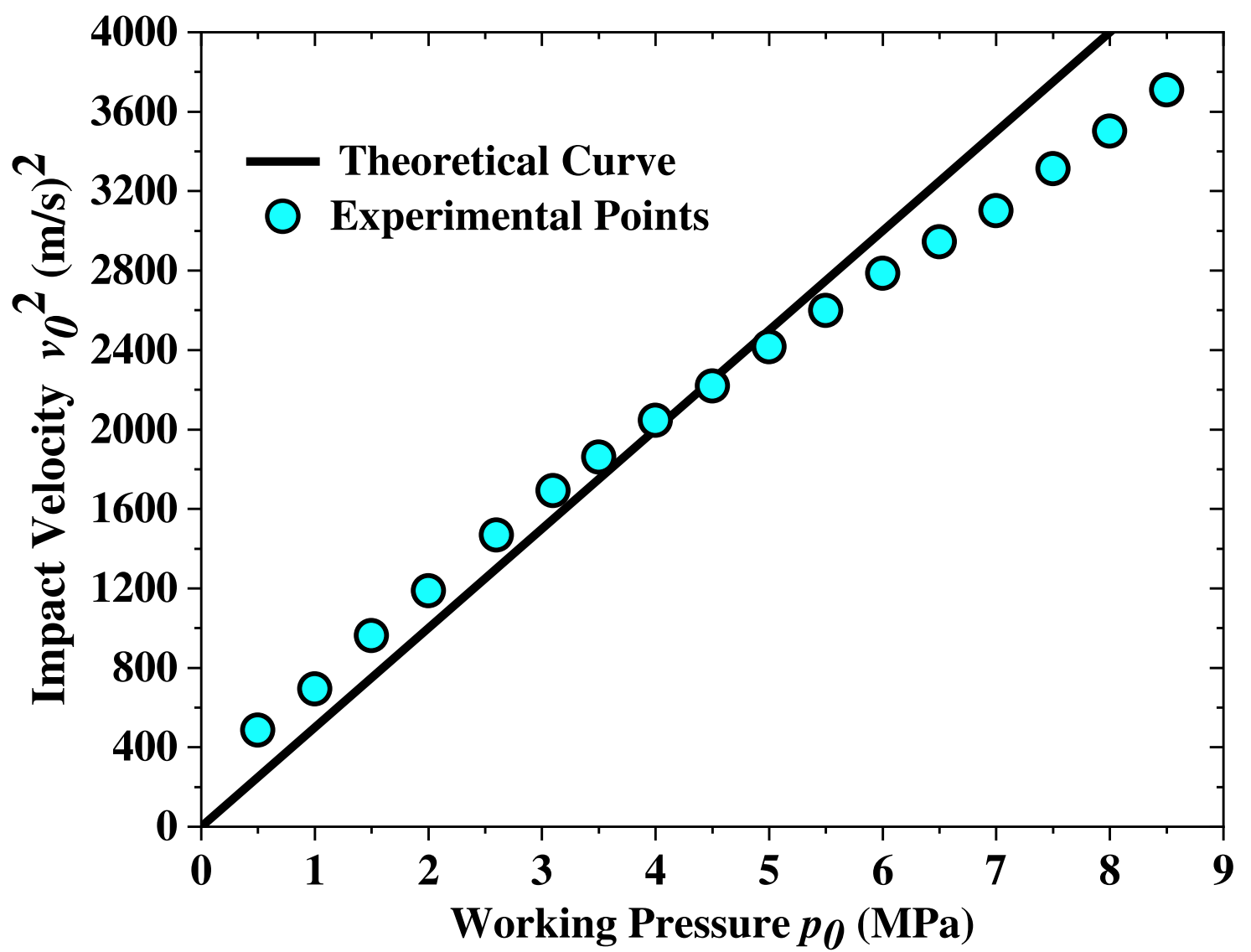

Fig.3. The relationship between initial launch velocity $v_{0}^{2}$ of gun barrel and working pressure $p_{0}$ 


\section{$50 \mathrm{~mm}$}

Finite Elements Interface Elements
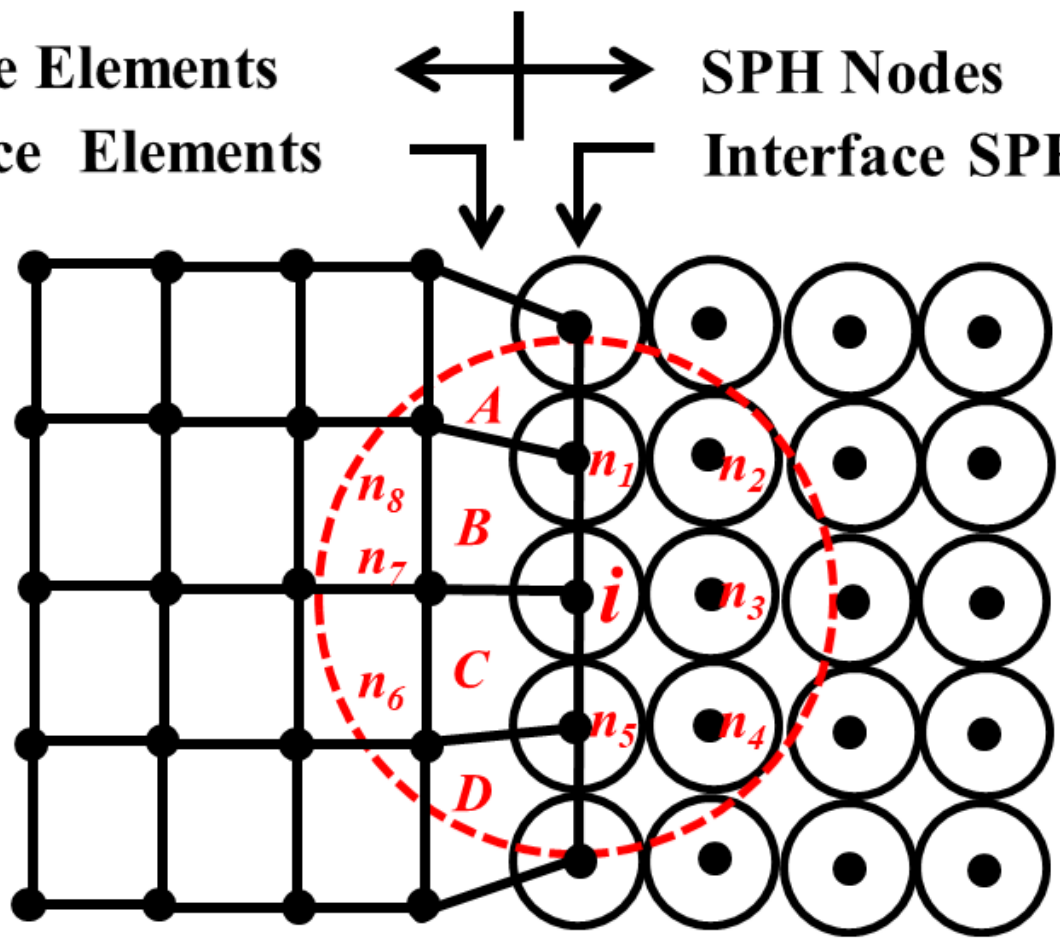

(a) SPH nodes attachment to grid without background particles

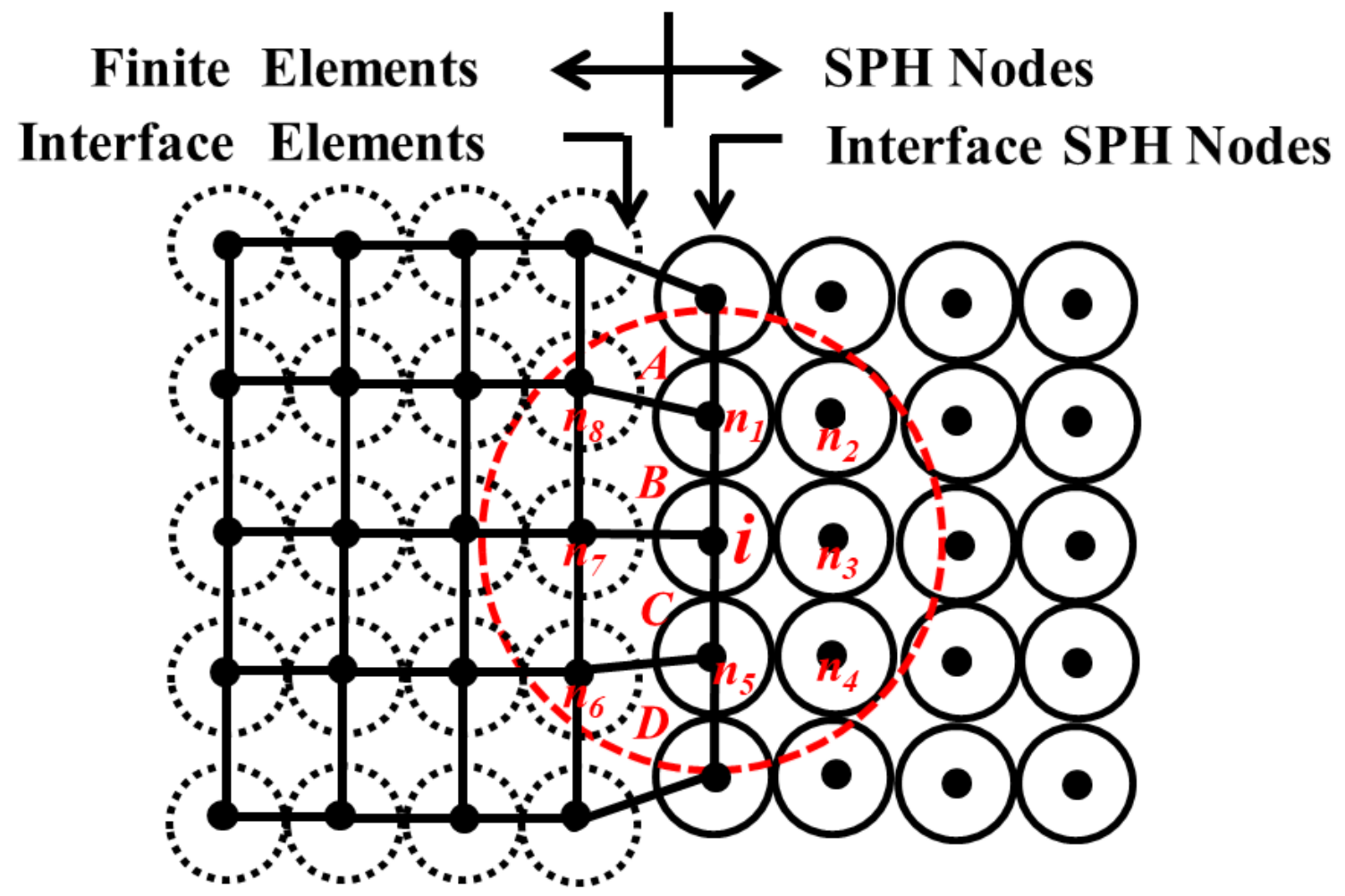

(b) SPH nodes attachment to grid with background particles Fig.4. SPH particles attached to finite elements 


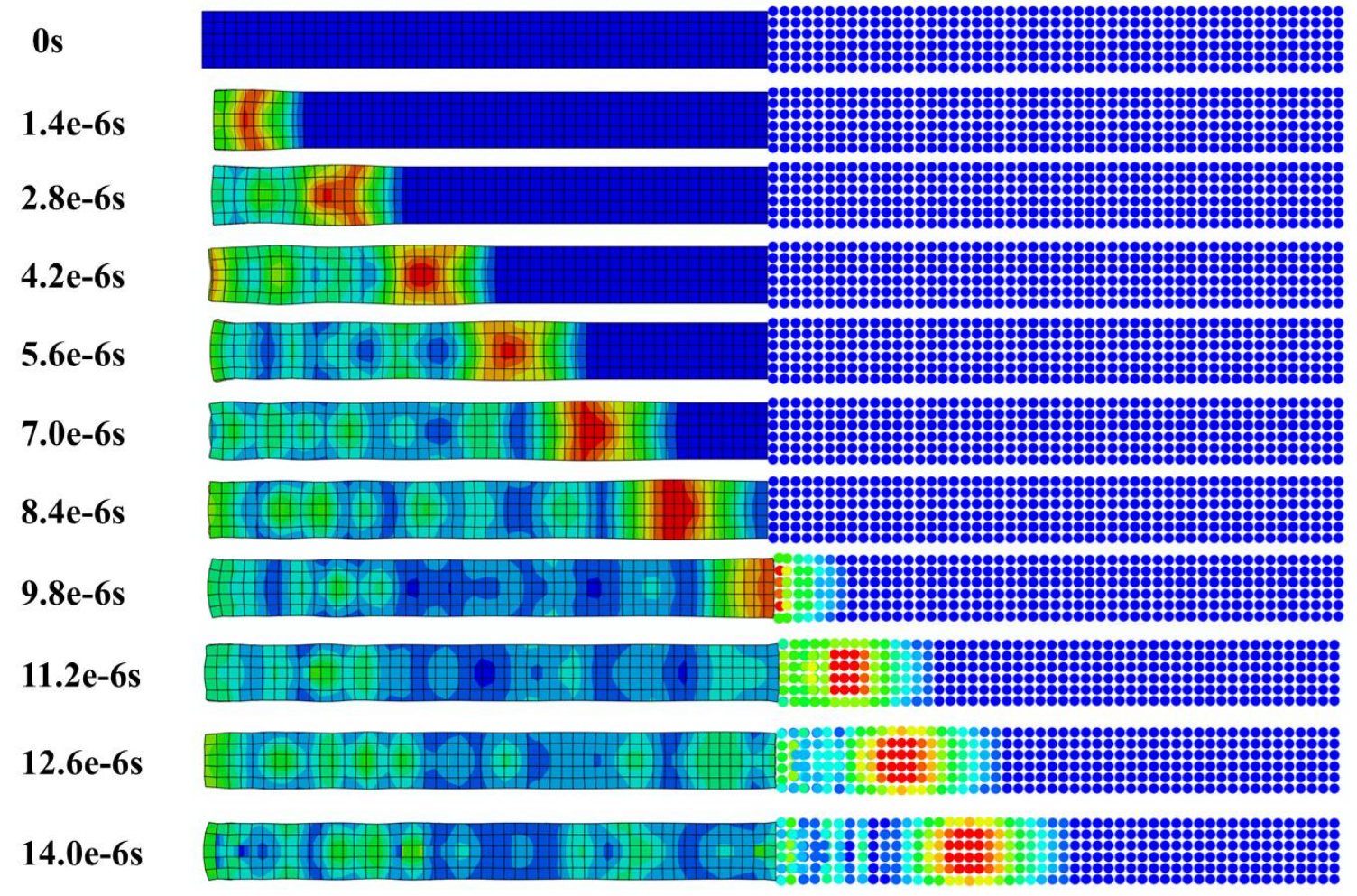

(a) Stress wave propagation of FEM-SPH coupled model

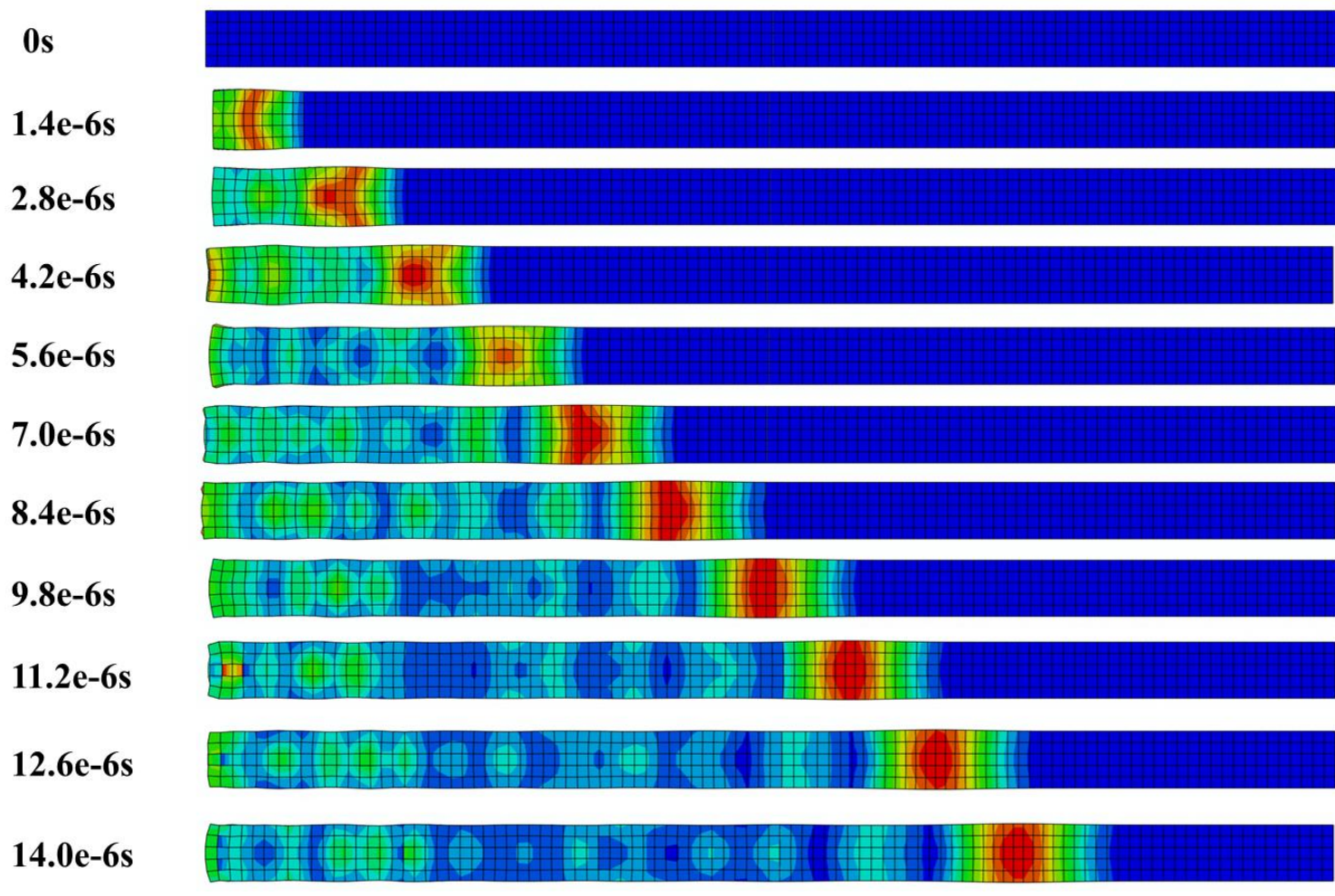

(b) Stress wave propagation of FEM model

Fig.5. Comparison of stress wave propagation between FEM-SPH model and FEM model 


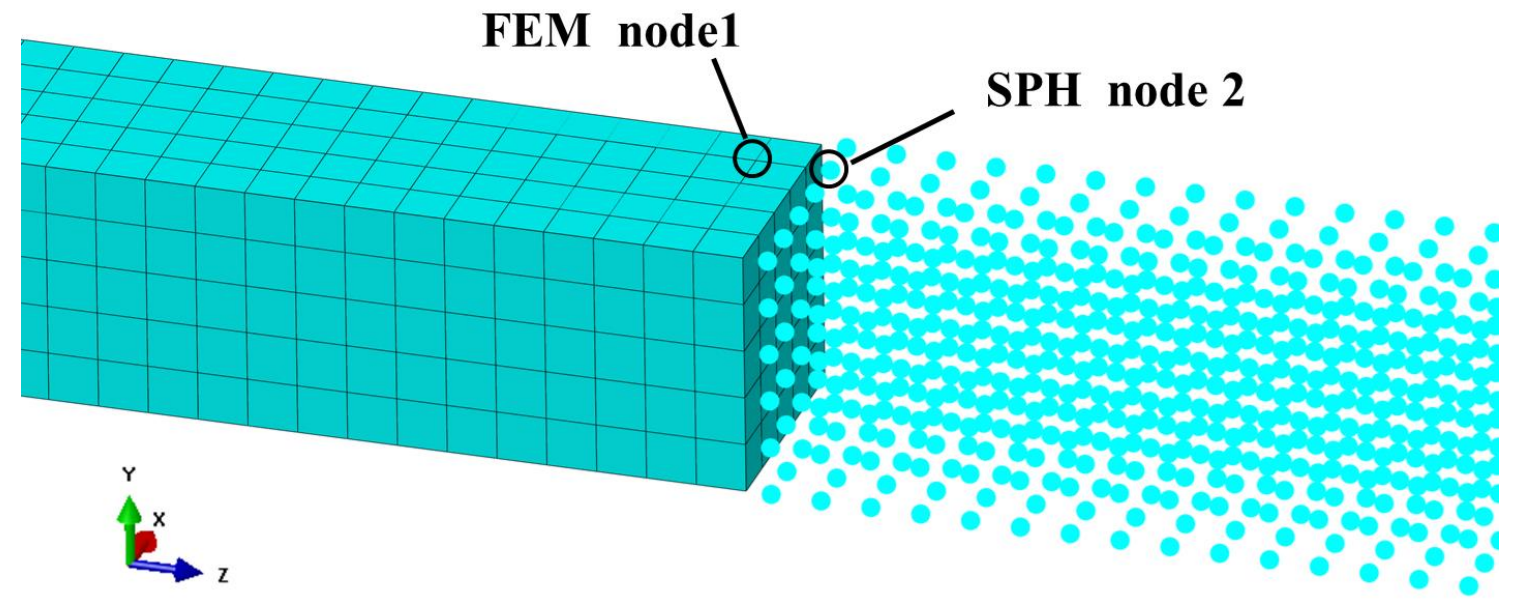

(a) Reference points on FEM-SPH coupled model

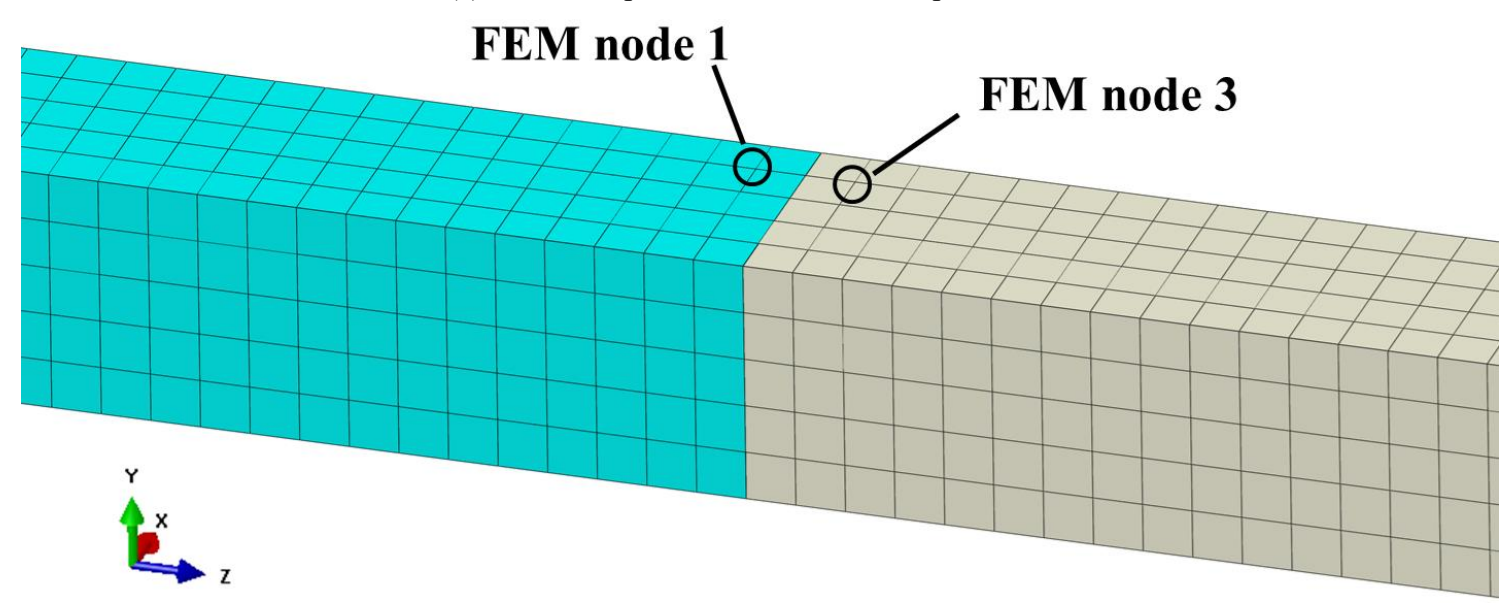

(b) Reference points on FEM model

Fig.6. Labeling of reference finite element nodes and SPH particles 


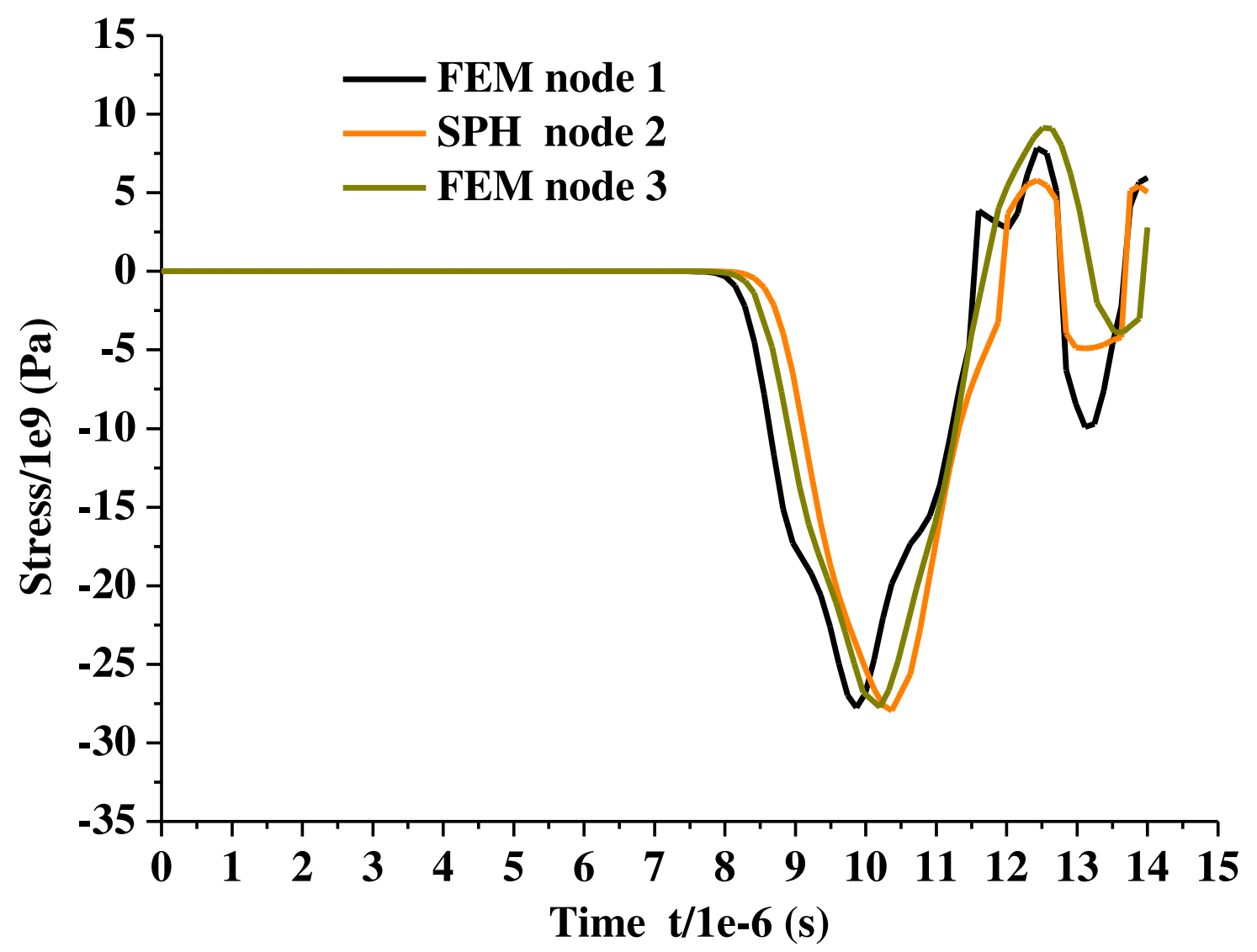

(a) The relationship of stress change with time

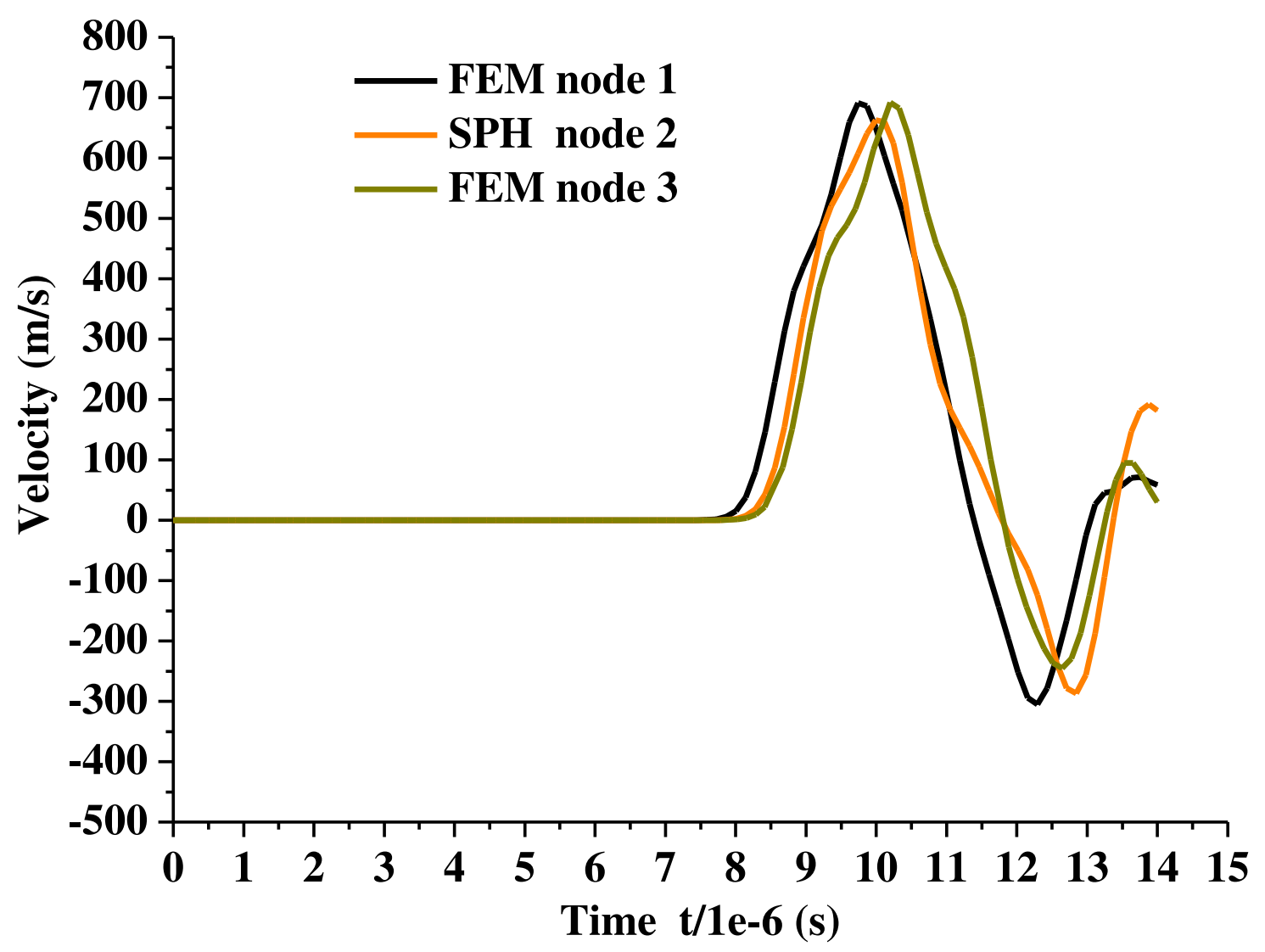

(b) The relationship of velocity change with time

Fig.7. Field variables of three reference points change with time 


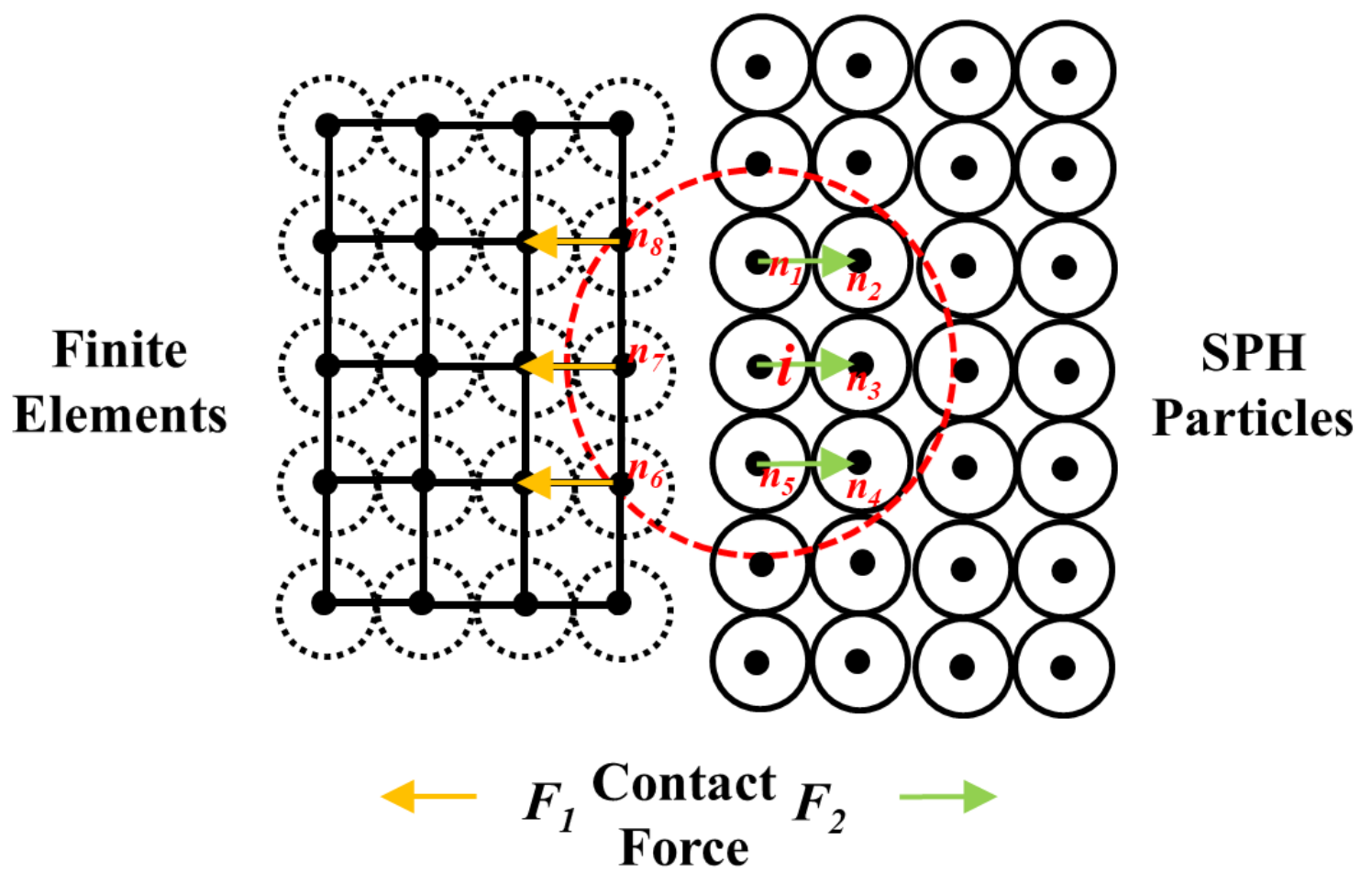

Fig.8. SPH particles contact with finite elements 


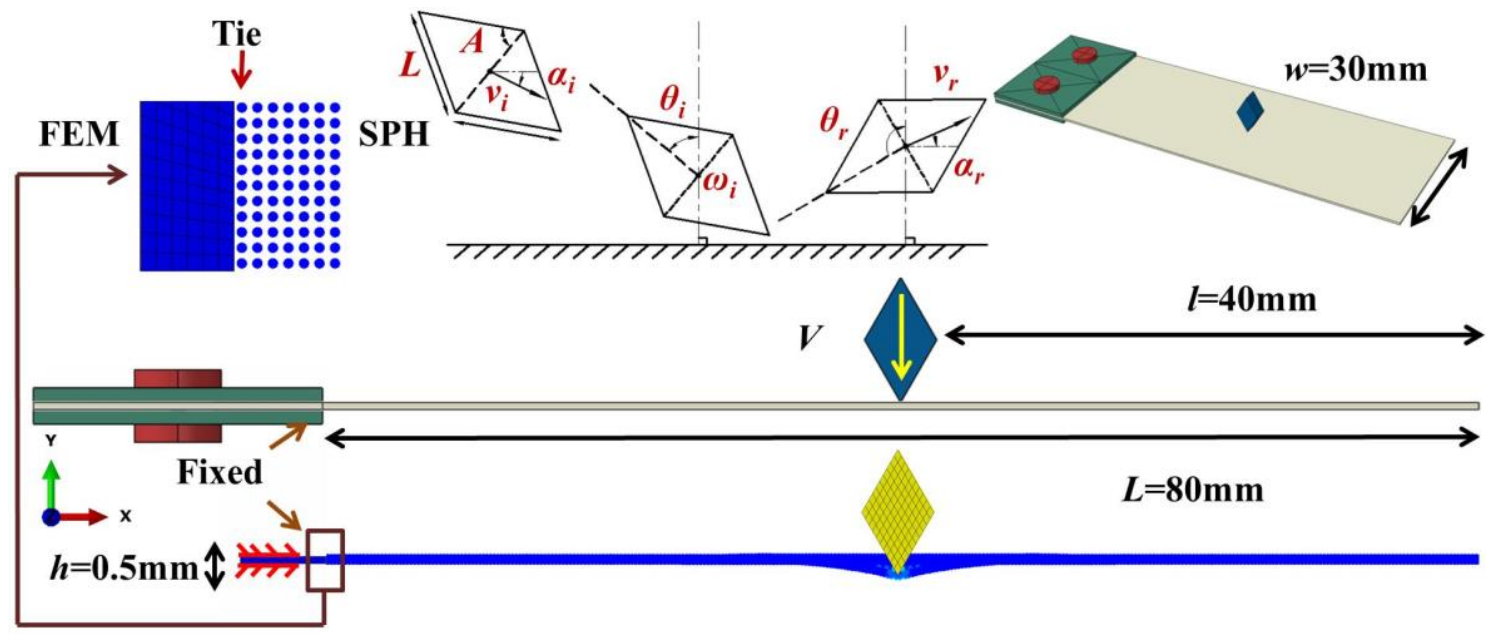

(a) Rhomboid shaped particle impacting cantilever beam

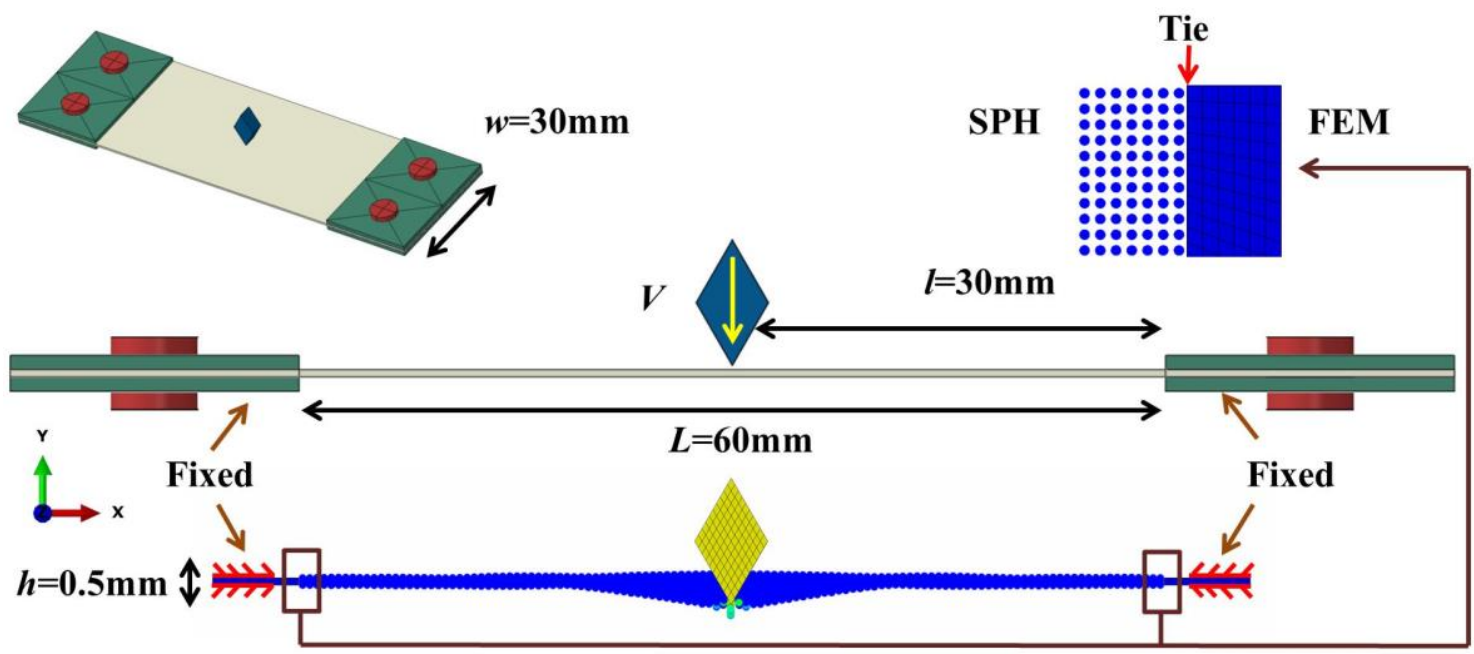

(b) Rhomboid shaped particle impacting fixed-fixed beam

Fig.9. Coupled FEM-SPH numerical model of rhomboid-shaped particle impacts Al6061-T6 thin sheet 

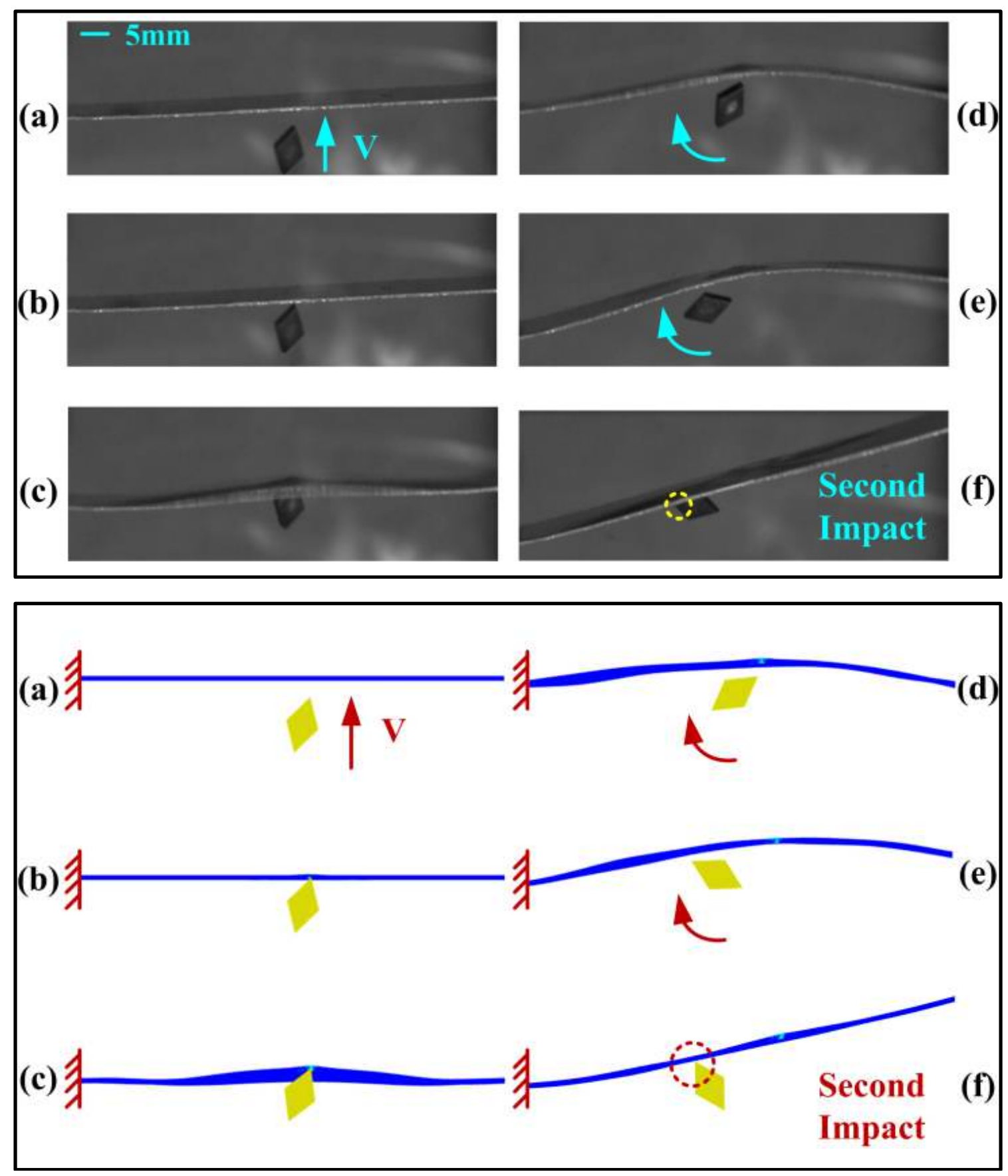

(a) Rhomboid-shaped particle impacting on cantilever beam: $\mathrm{V}=36.75 \mathrm{~m} / \mathrm{s}, \alpha_{i}=90^{\circ}, \theta_{i}=-16^{\circ}$ 

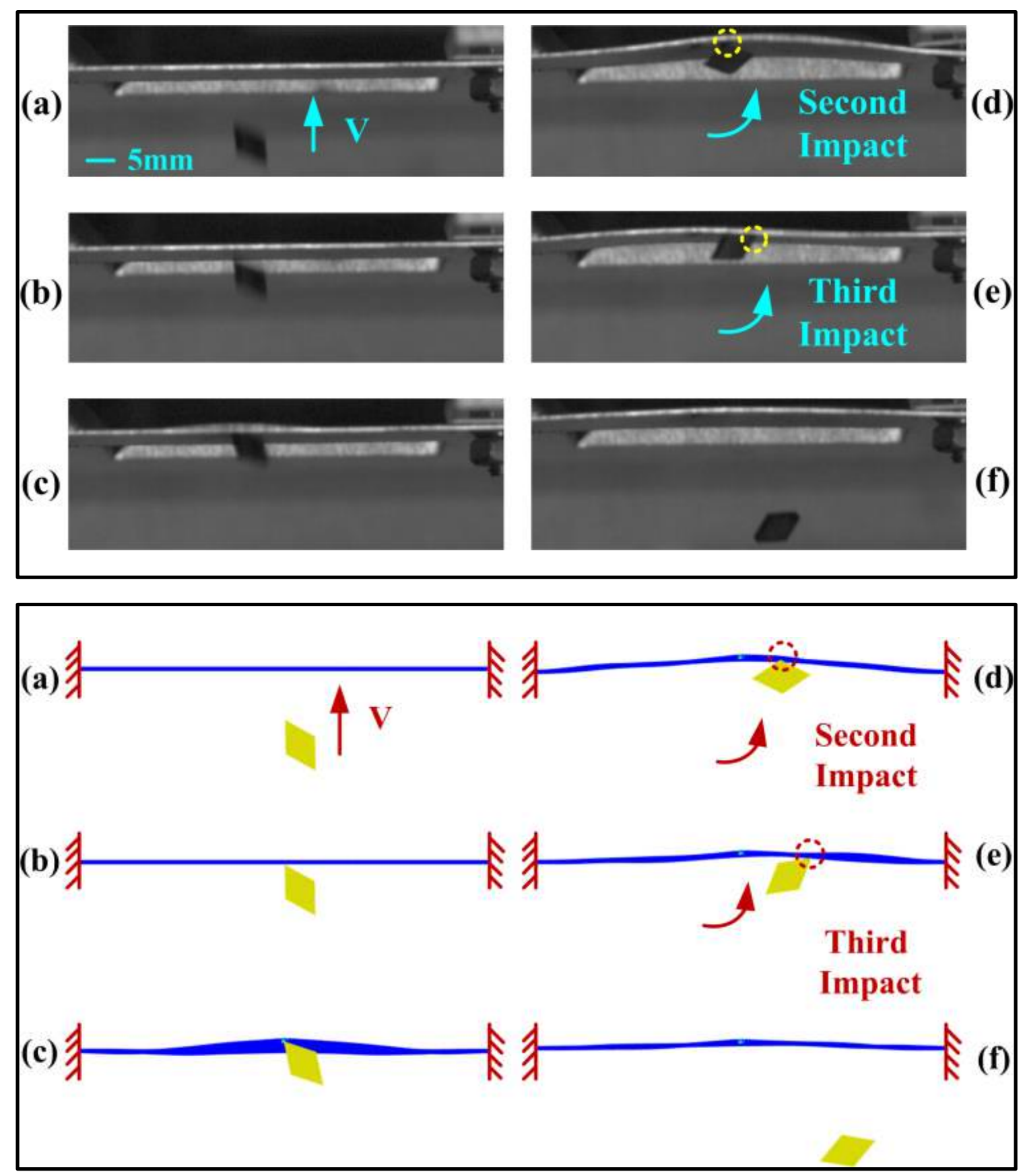

(b) Rhomboid-shaped particle impacting on fixed-fixed-beam: $\mathrm{V}=34.75 \mathrm{~m} / \mathrm{s}, \alpha_{i}=90^{\circ}, \theta_{i}=31^{\circ}$

Fig.10. Comparison of experimental and simulated results of rhomboid-shaped particle impacting on A16061-T6 thin plate 

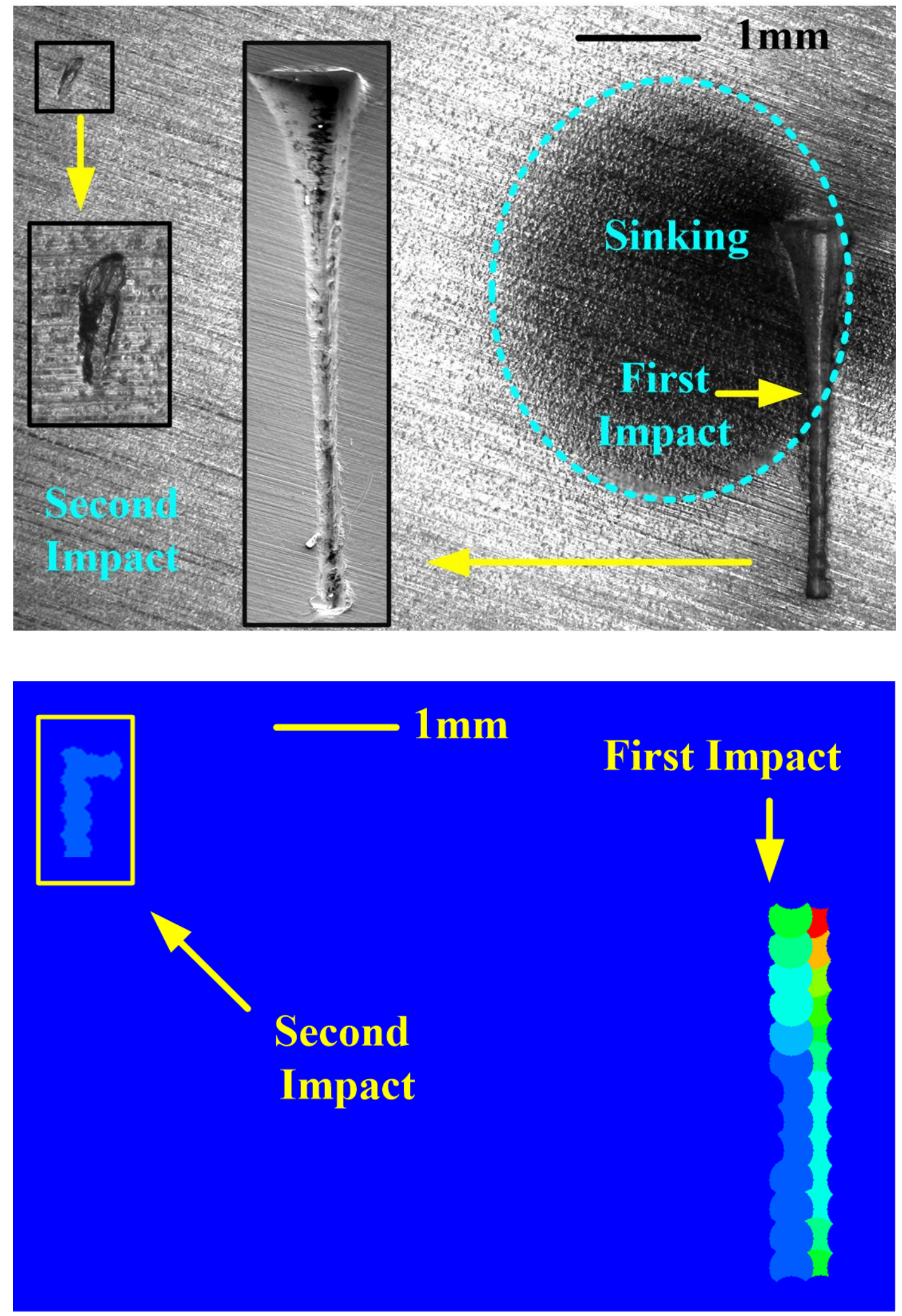

(a) Contour of craters on cantilever beam formed by rhomboid-shaped particle 

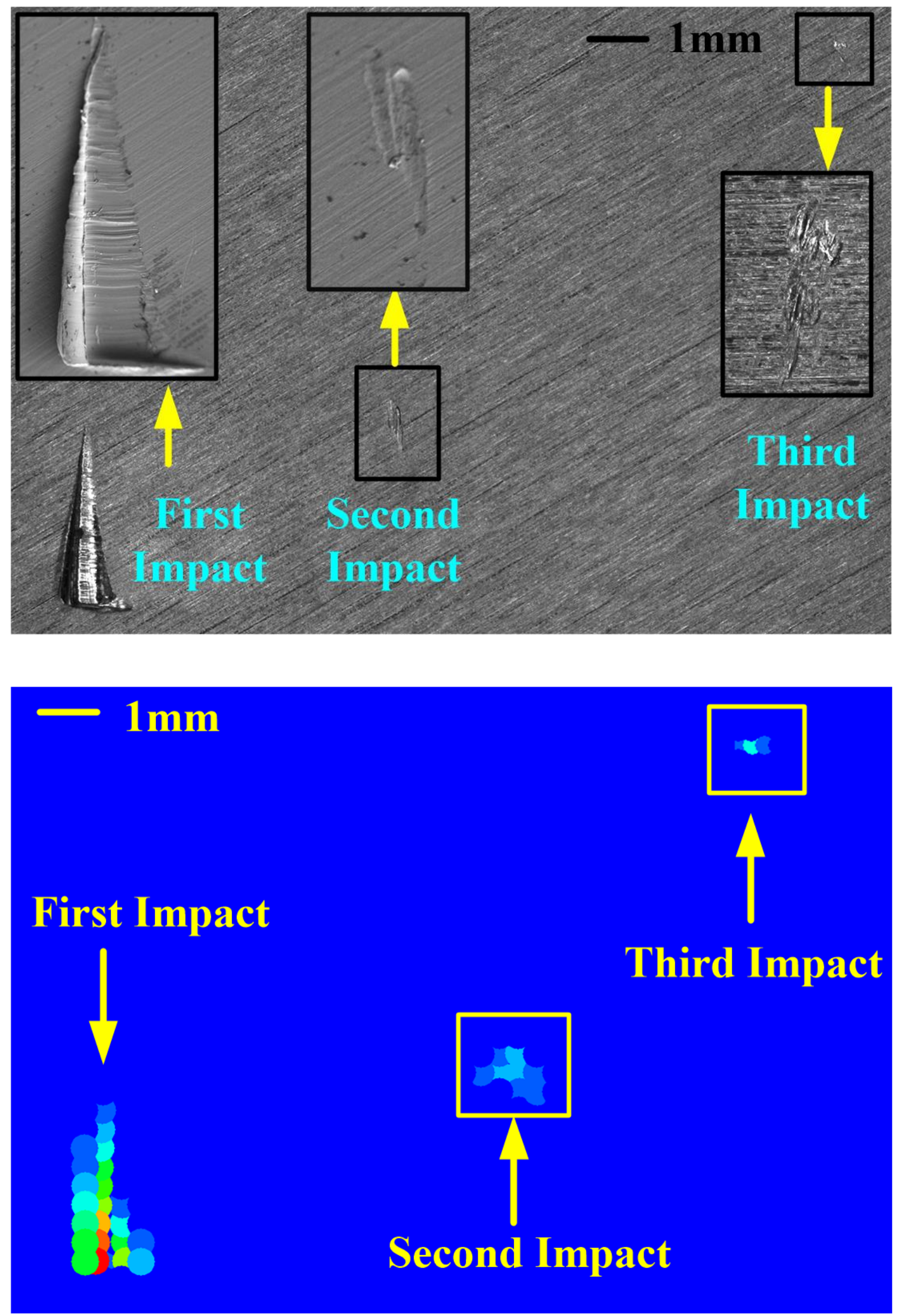

(b) Contour of craters on fixed-fixed beam formed by rhomboid-shaped particle

Fig.11. Comparison of experimental and simulated results of craters profile formed by rhomboid-shaped particle 


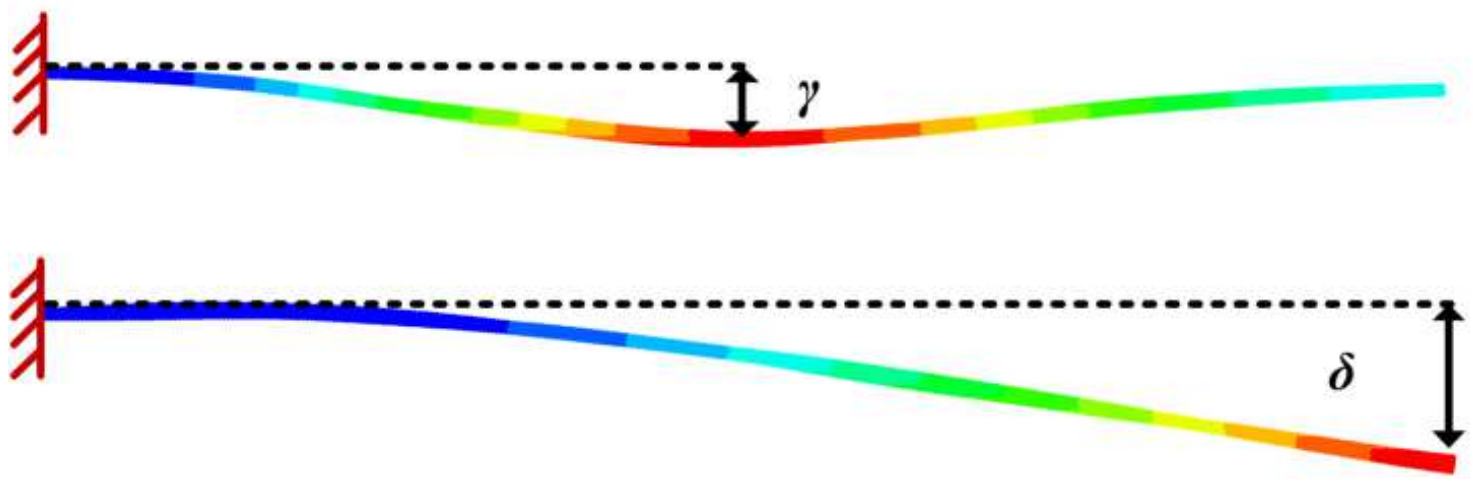

(a) Deformation deflection diagram of cantilever beam
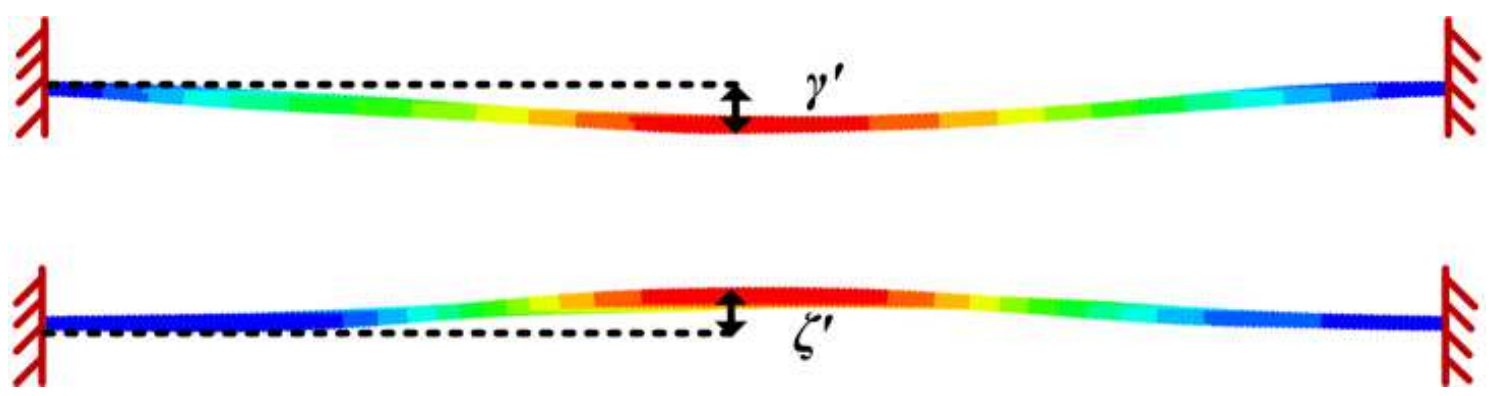

(b) Deformation deflection diagram of fixed-fixed-beam

Fig.12. Deflection diagram of cantilever beam and fixed-fixed beam after being impacted by particles $\left(\mathrm{V}=20 \mathrm{~m} / \mathrm{s}, \alpha_{i}=90^{\circ}, \theta_{i}=0^{\circ}\right)$ 


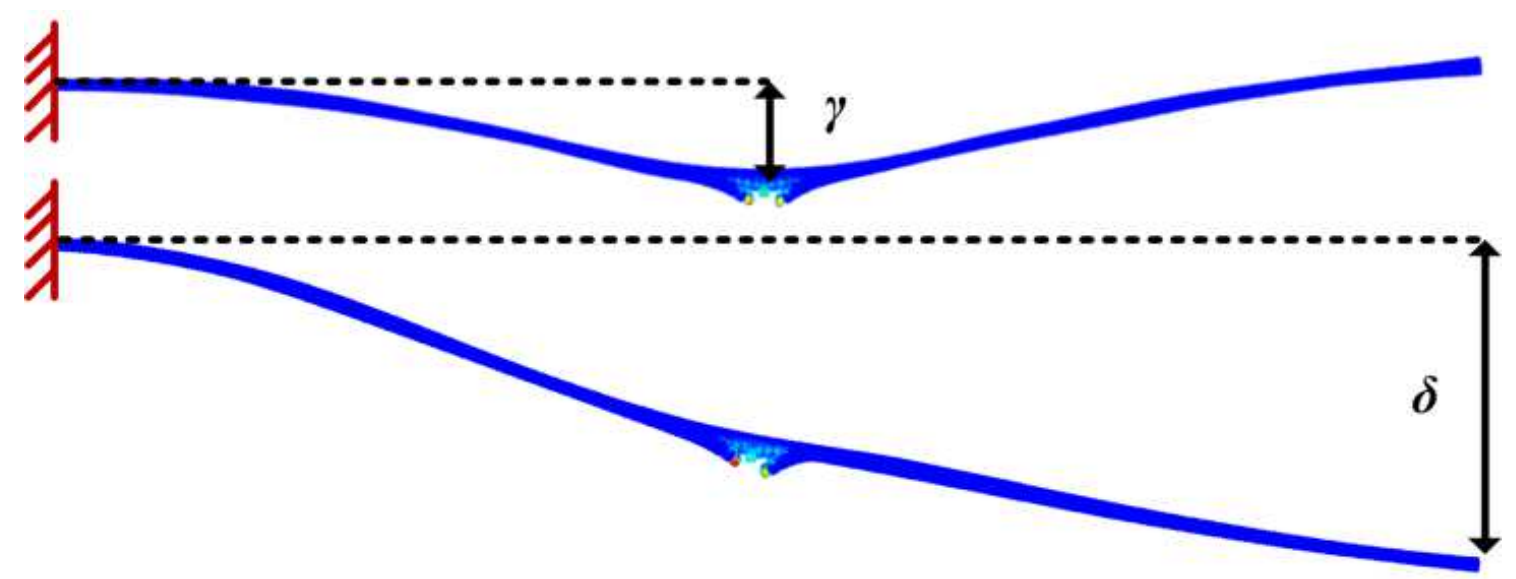

(a) Plastic bending and breakthrough of cantilever beam
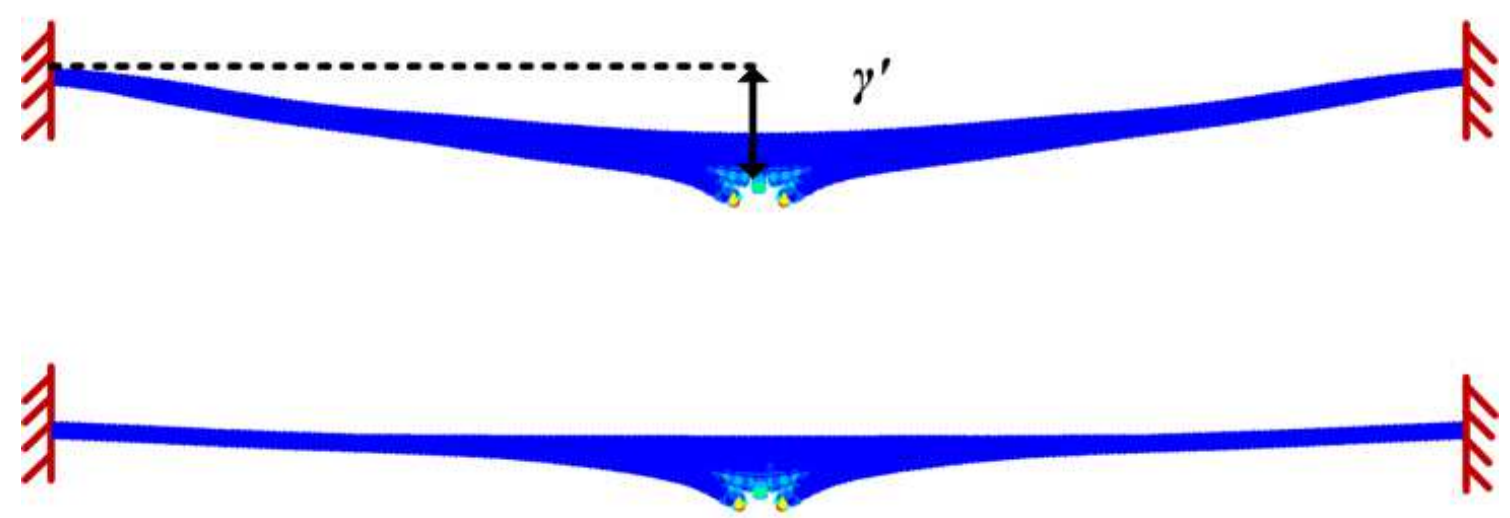

(b) Plastic bending and breakthrough of fixed-fixed beam

Fig.13. Plastic bending and breakthrough of thin plate (cantilever beam \& fixed-fixed beam) after impacting by rhomboid-shaped particle under medium-high velocity $\left(\mathrm{V}=60 \mathrm{~m} / \mathrm{s}, \alpha_{i}=90^{\circ}, \theta_{i}=0^{\circ}\right)$ 

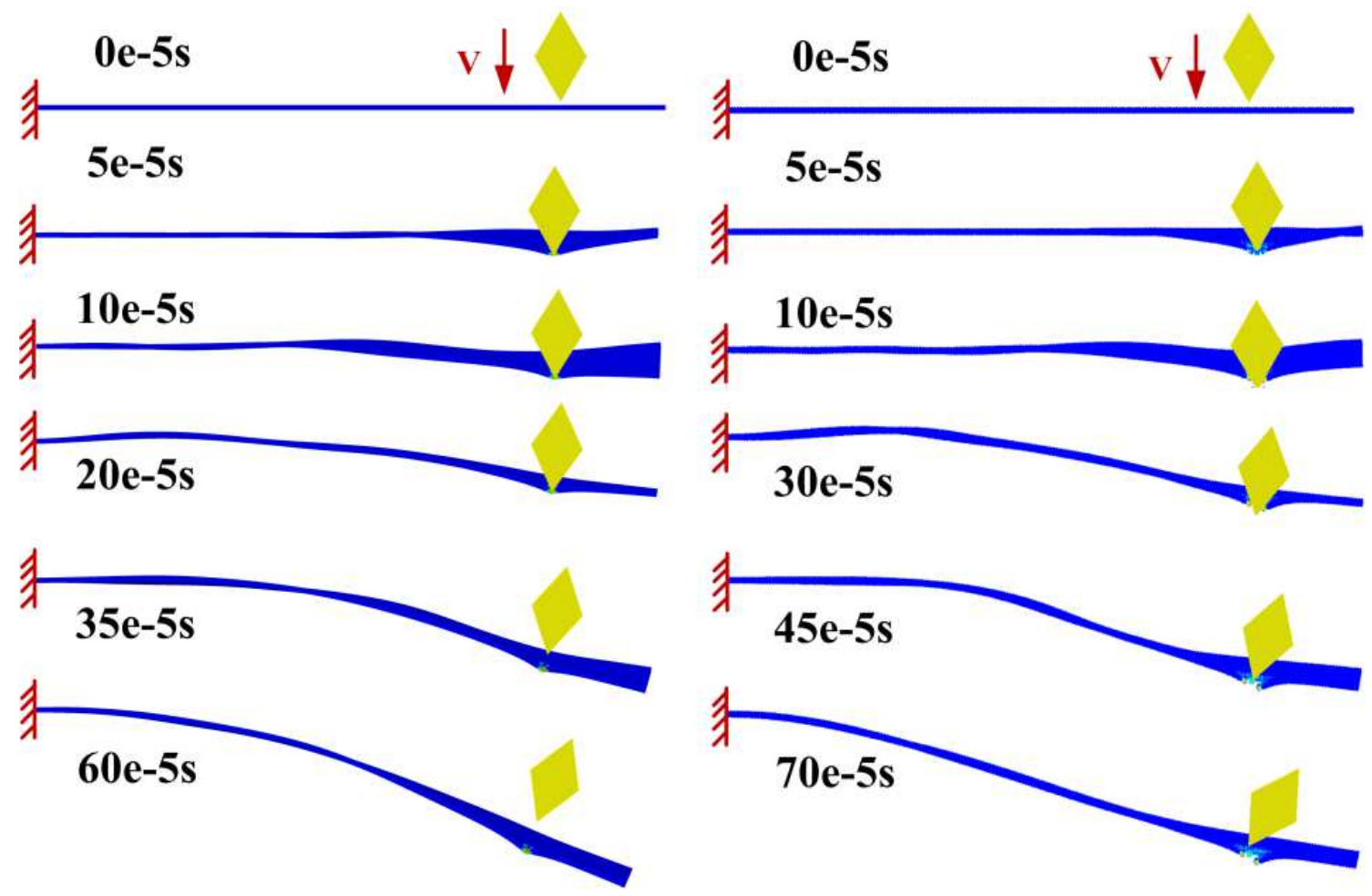

(a) Simulated results of FEM model

(b) Simulated results of FEM-SPH coupled model

Fig.14. Comparison and verification of different models to capture substrate deformation under medium-high velocity impact conditions $\left(\mathrm{V}=60 \mathrm{~m} / \mathrm{s}, \alpha_{i}=90^{\circ}, \theta_{i}=0^{\circ}\right)$ 


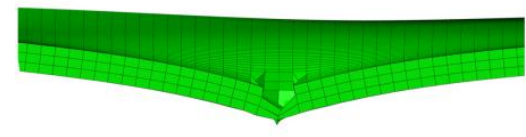

(a) Crater predicted by FEM model

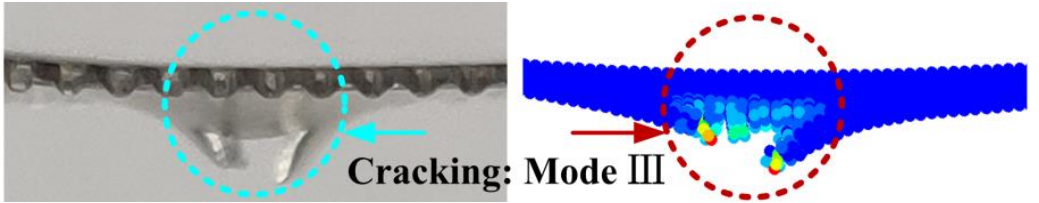

$\begin{array}{ll}\text { (b) Crater from experiments } & \text { (c) Crater predicted by FEM-SPH coupled model }\end{array}$

Fig.15. Comparison and verification of different models to capture substrate breakdown under medium-high velocity impact conditions 


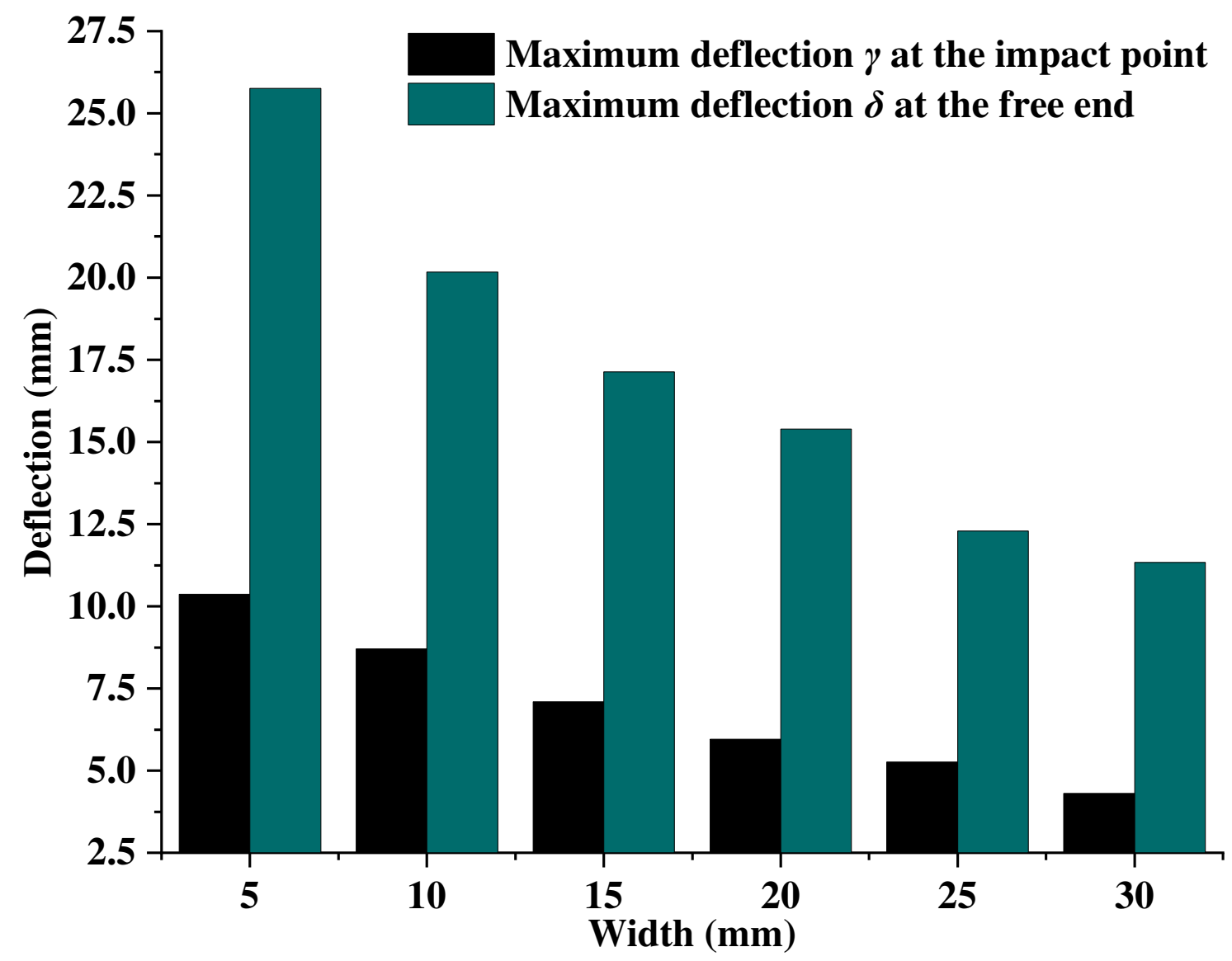

Fig. 16 Variation of maximum deflection $\gamma$ and $\delta$ of cantilever beam with beam width w $\left(\mathrm{V}=30 \mathrm{~m} / \mathrm{s}, \alpha_{i}=90^{\circ}, \theta_{i}=0^{\circ}, L=60 \mathrm{~mm}, h=0.5 \mathrm{~mm}\right)$ 


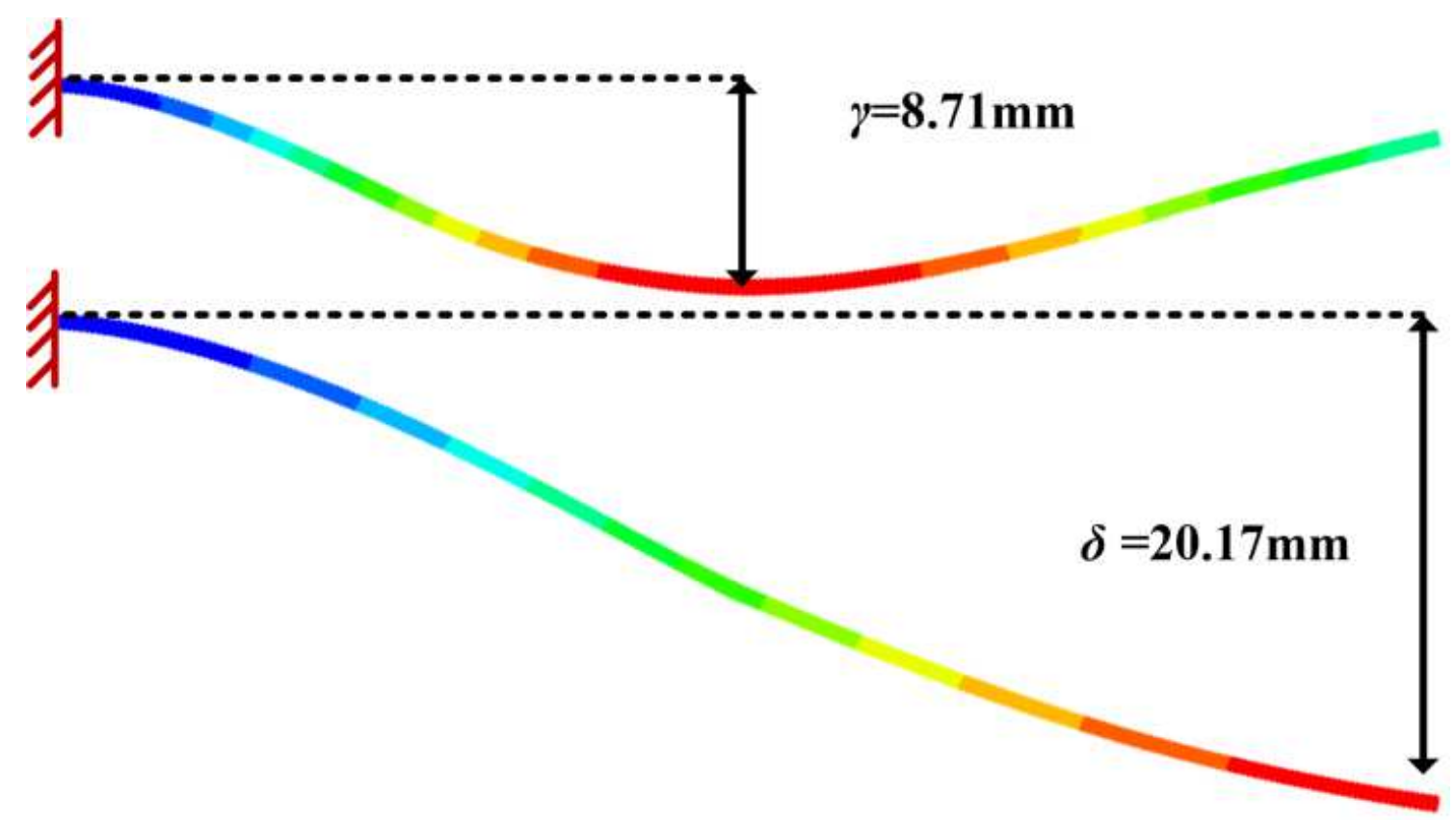

(a) The width of cantilever beam: $w=10 \mathrm{~mm}$
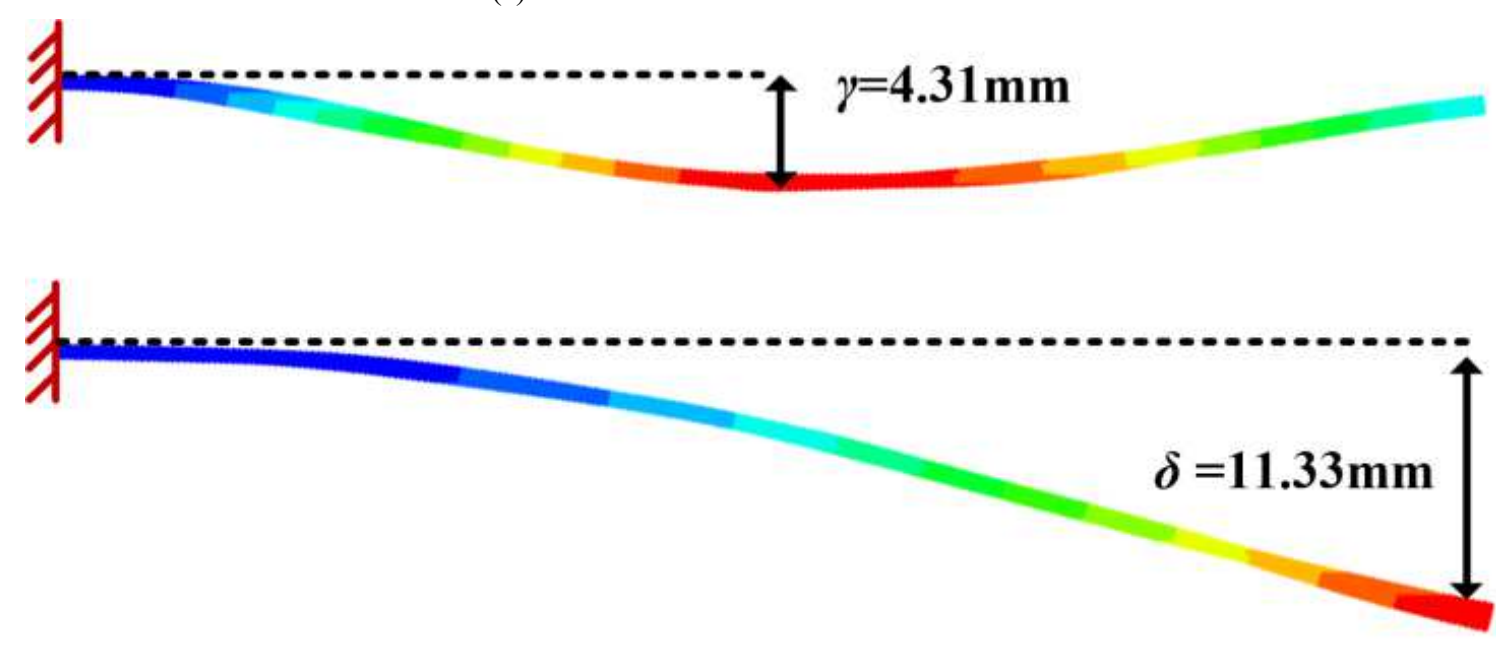

(b) The width of cantilever beam: $w=30 \mathrm{~mm}$

Fig.17. Deformation behavior of cantilever beams with different widths under the same impact condition $\left(\mathrm{V}=30 \mathrm{~m} / \mathrm{s}, \alpha_{i}=90^{\circ}, \theta_{i}=0^{\circ}\right)$ 


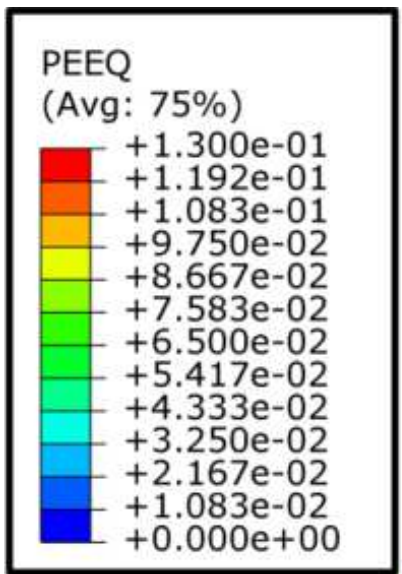

(a) The width of cantilever beam: $w=10 \mathrm{~mm}$
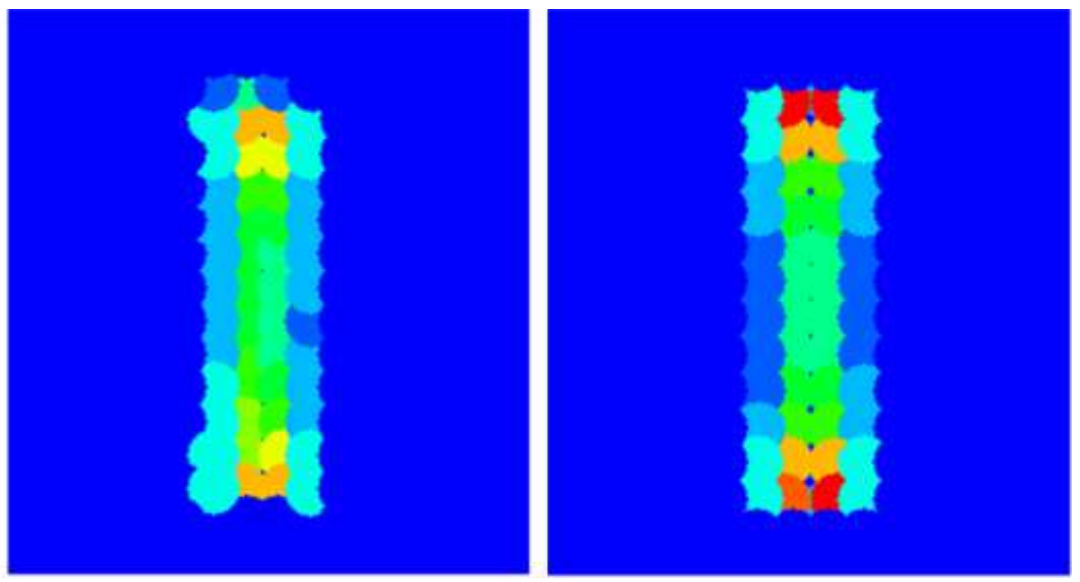

(b) The width of cantilever beam: $w=30 \mathrm{~mm}$

Fig.18. Crater contour of cantilever beams with different widths under the same impact condition $\left(\mathrm{V}=30 \mathrm{~m} / \mathrm{s}, \alpha_{i}=90^{\circ} \theta_{i}=0^{\circ}\right)$ 


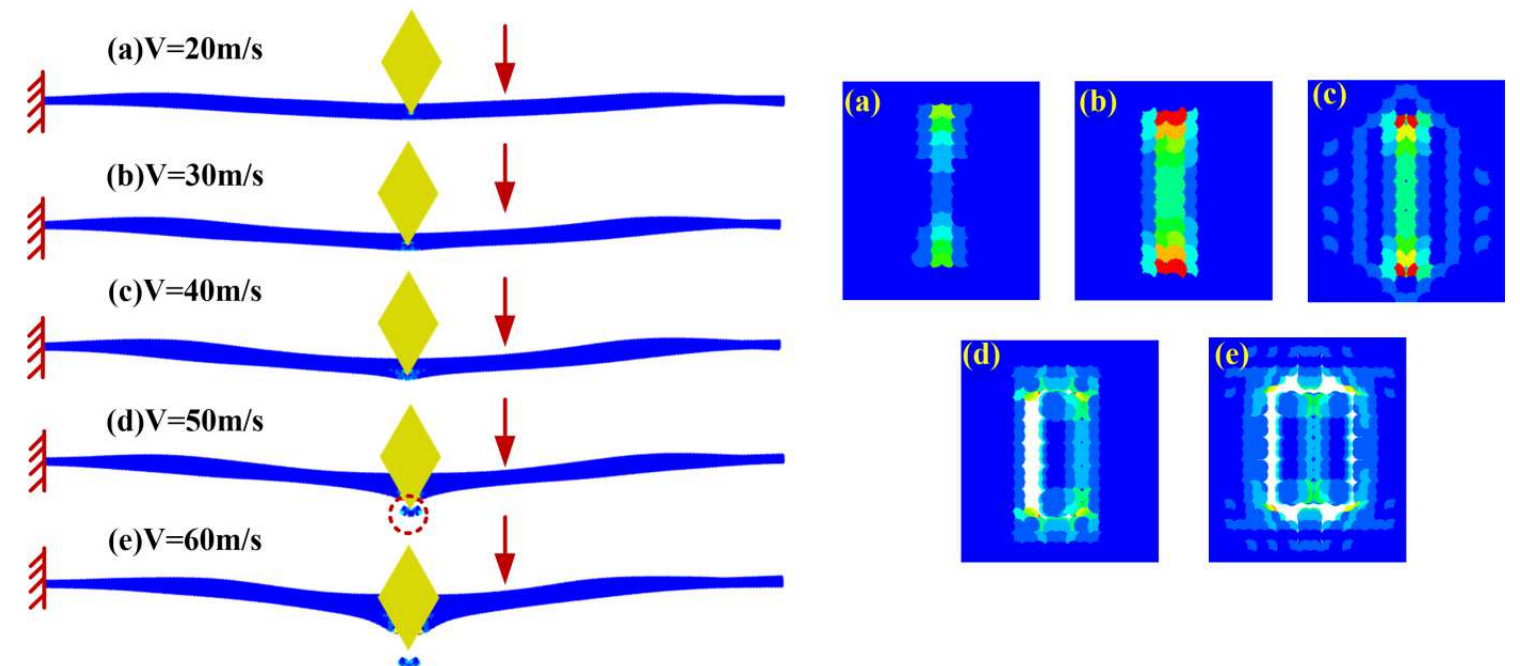

Fig.19. Deformation behavior and crater profiles of cantilever beam at different incident velocities $(l=60 \mathrm{~mm}, w=30 \mathrm{~mm}, h=0.5 \mathrm{~mm})$ 


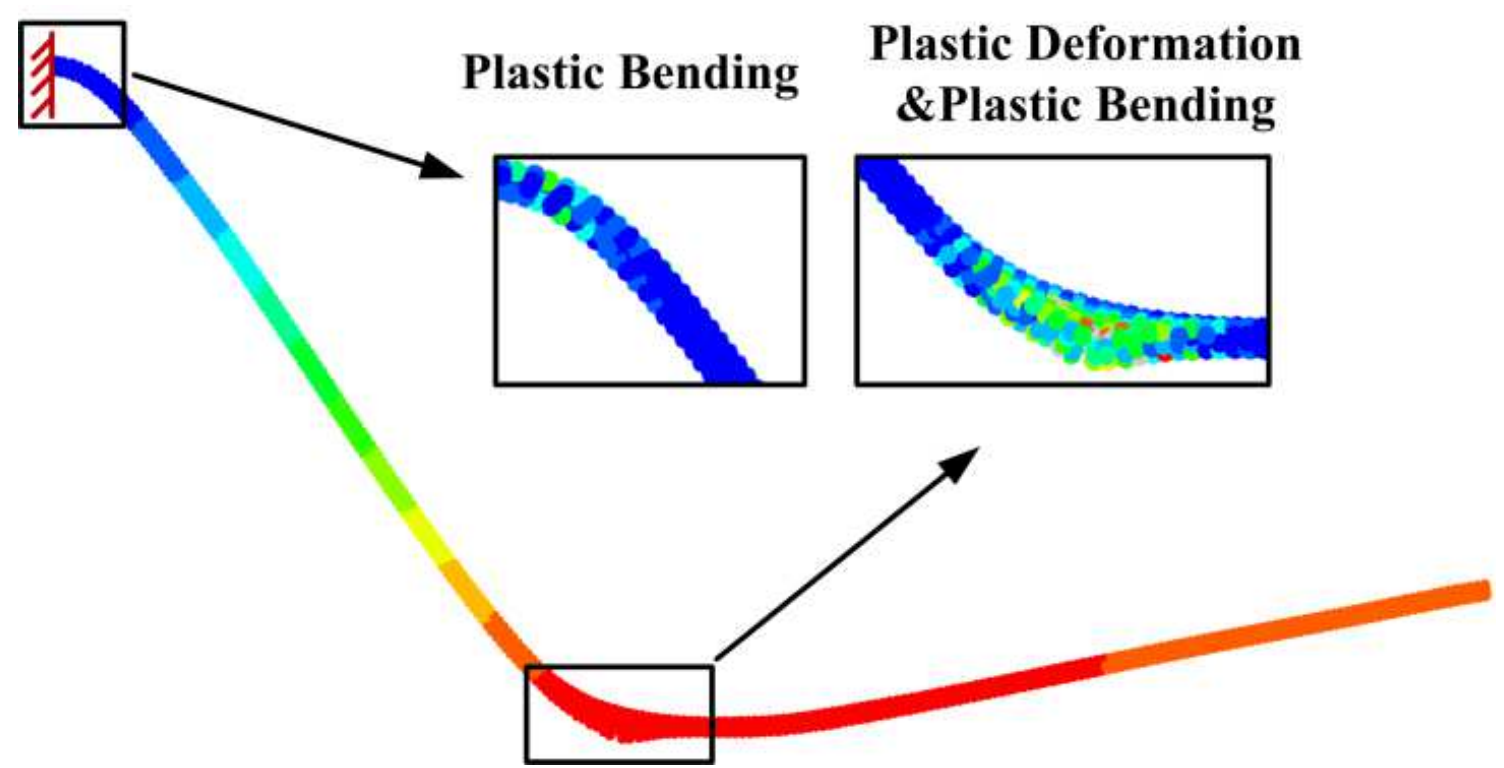

(a) The width of cantilever beam: $w=10 \mathrm{~mm}$

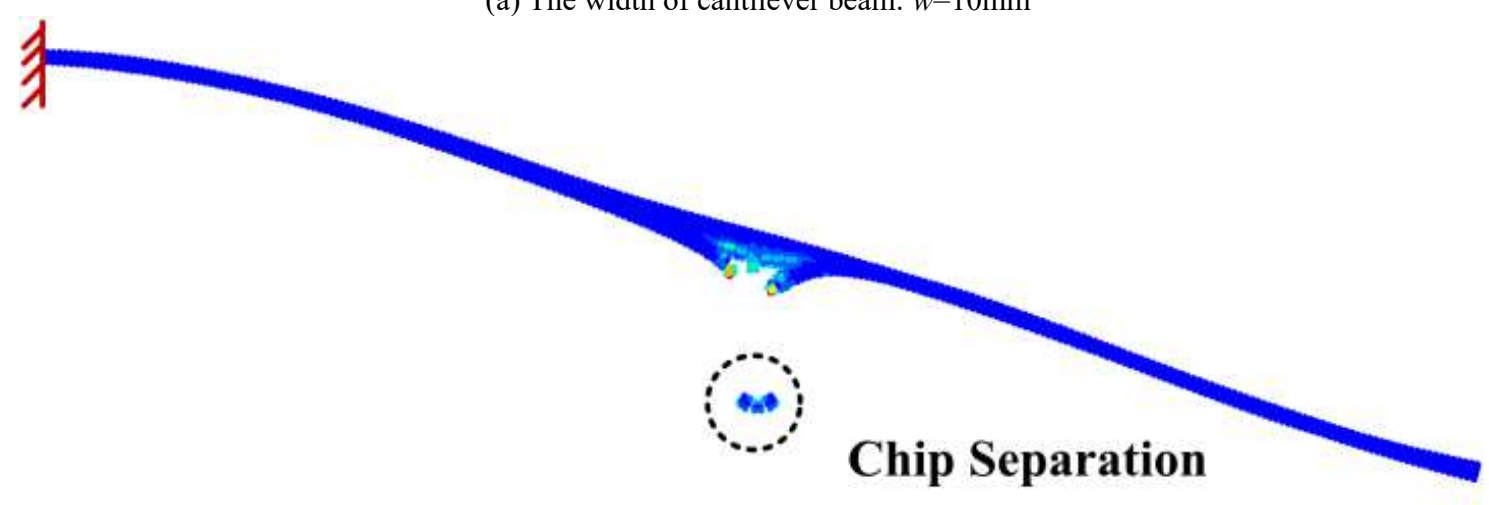

(b) The width of cantilever beam: $w=30 \mathrm{~mm}$

Fig.20. Deformation behavior of cantilever beams with different widths under the same impact condition $\left(\mathrm{V}=60 \mathrm{~m} / \mathrm{s}, \alpha_{i}=90^{\circ}, \theta_{i}=0^{\circ}\right)$ 


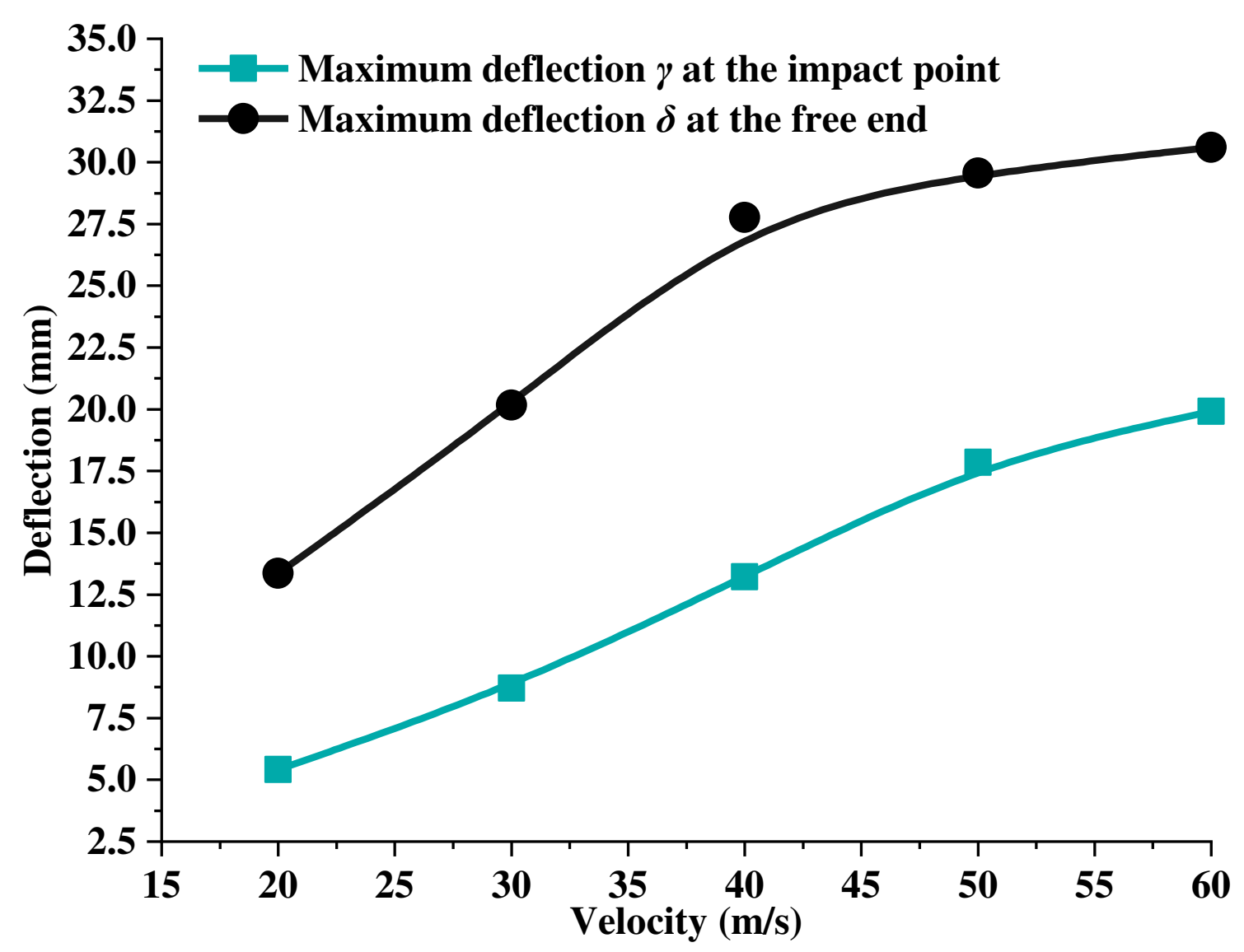

(a) The size of cantilever beam: $L=60 \mathrm{~mm}, \mathrm{w}=10 \mathrm{~mm}, \mathrm{~h}=0.5 \mathrm{~mm}$

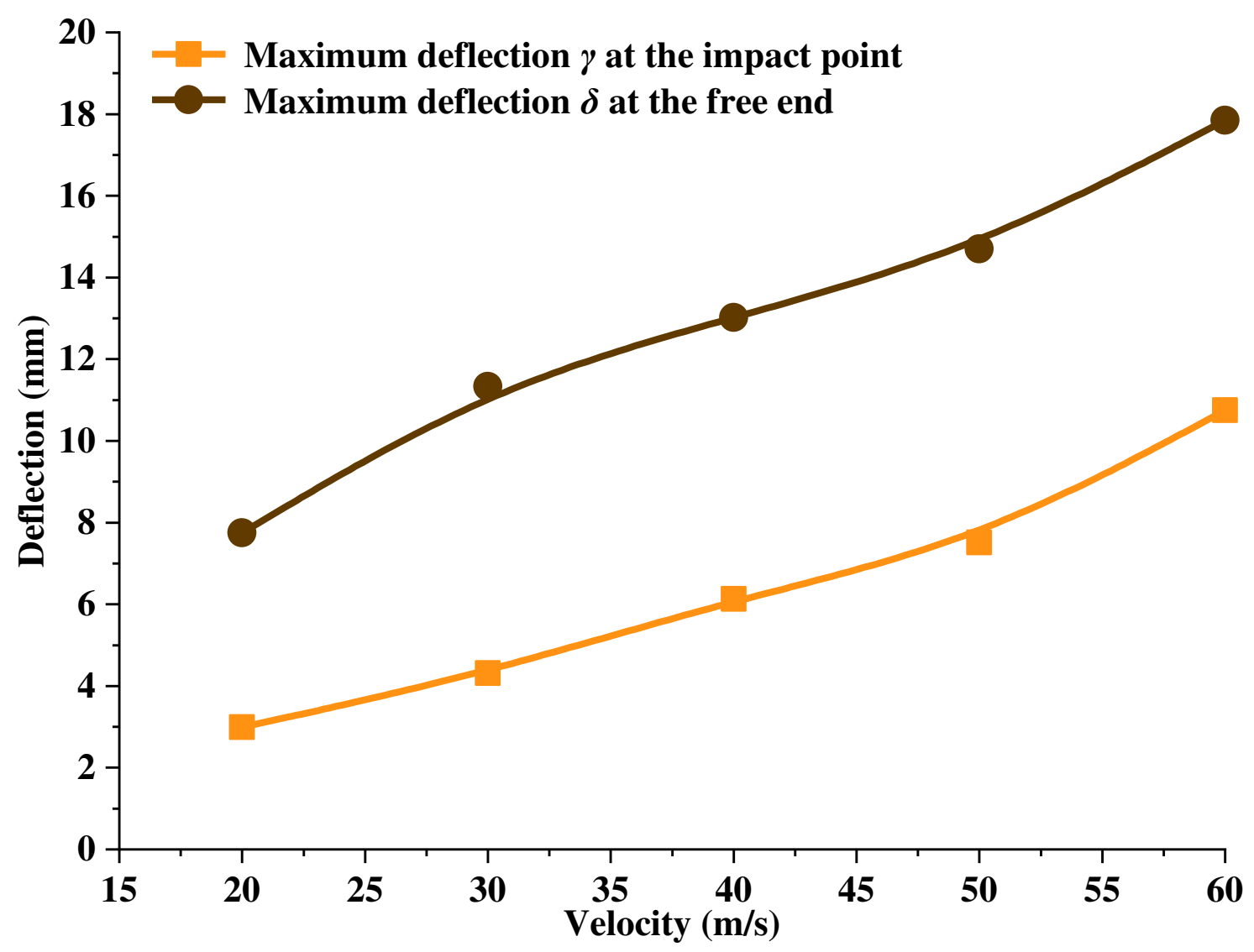

(b) The size of cantilever beam: $L=60 \mathrm{~mm}, \mathrm{w}=30 \mathrm{~mm}, \mathrm{~h}=0.5 \mathrm{~mm}$

Fig.21. Variation of deflection of cantilever beams of different sizes with the incident velocity 

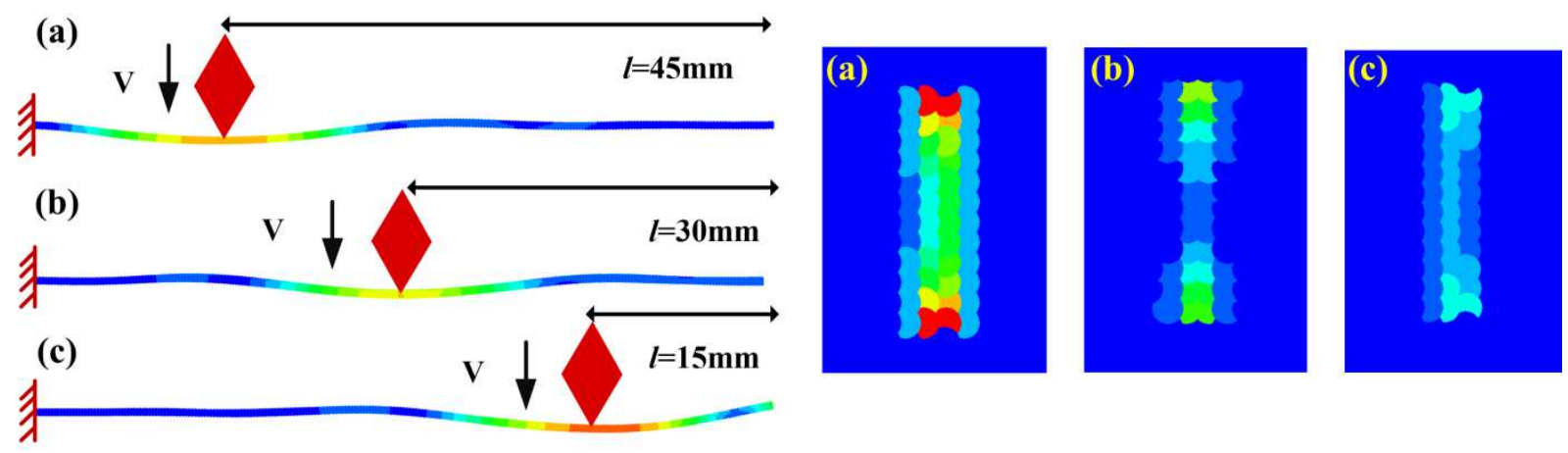

Fig.22. Different impact positions of cantilever beams and corresponding crater contours 


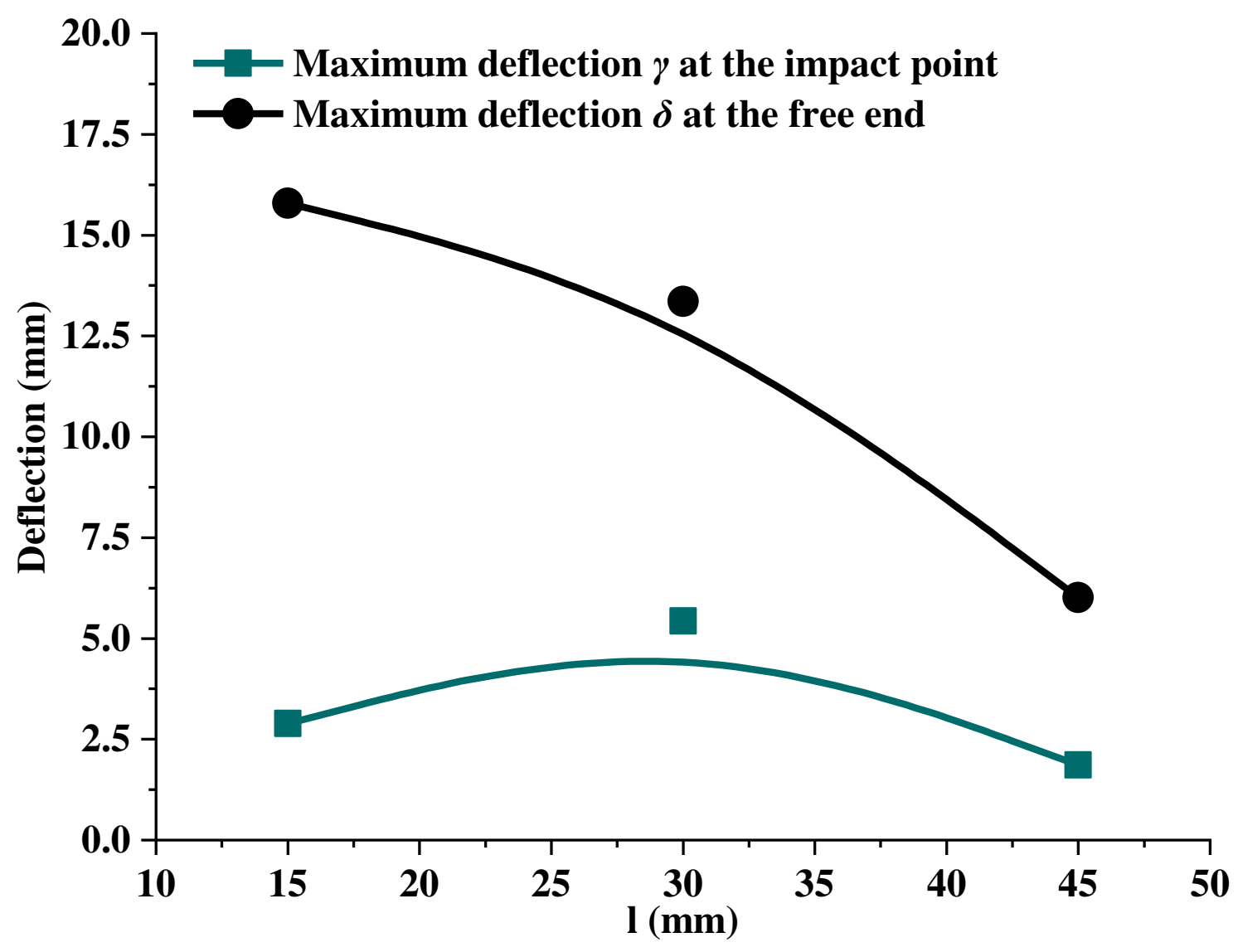

(a) The size of cantilever beam: $L=60 \mathrm{~mm}, \mathrm{w}=10 \mathrm{~mm}, \mathrm{~h}=0.5 \mathrm{~mm}$

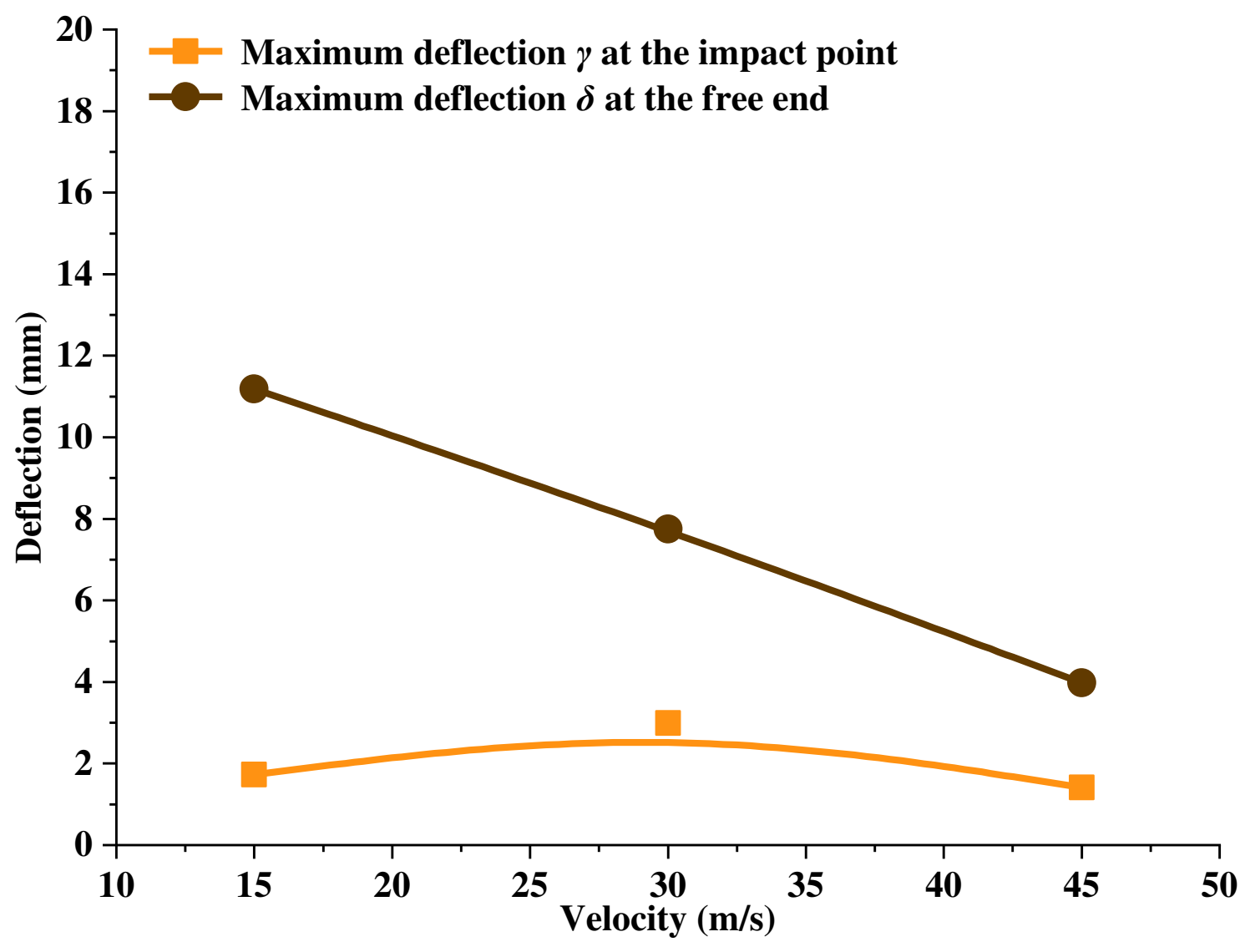

(b) The size of cantilever beam: $L=60 \mathrm{~mm}, \mathrm{w}=30 \mathrm{~mm}, \mathrm{~h}=0.5 \mathrm{~mm}$

Fig.23. Deflection of cantilever beams of different sizes varies with the incident position of particles 


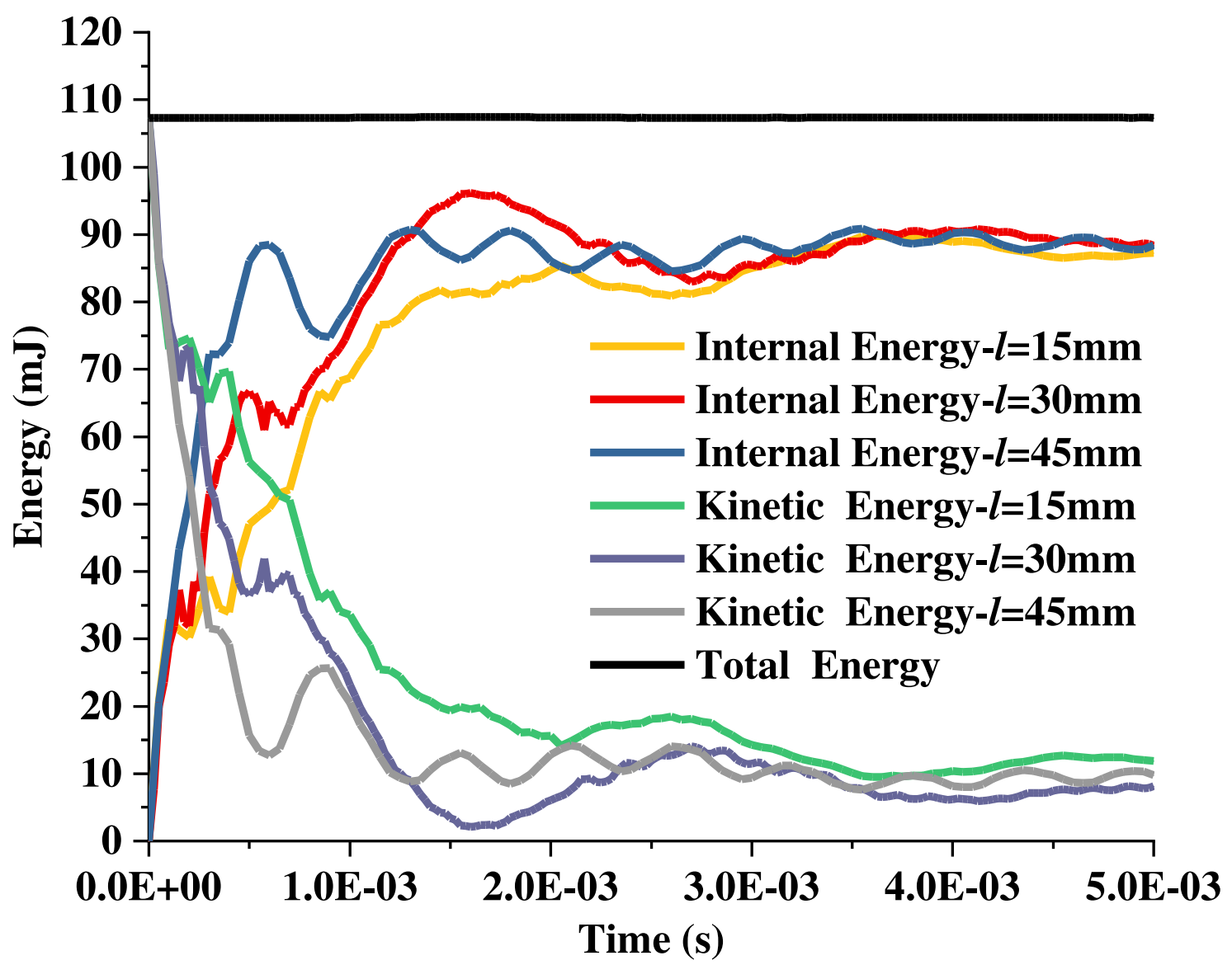

Fig.24. Energy evolution curves of cantilever beam system at different impact positions 

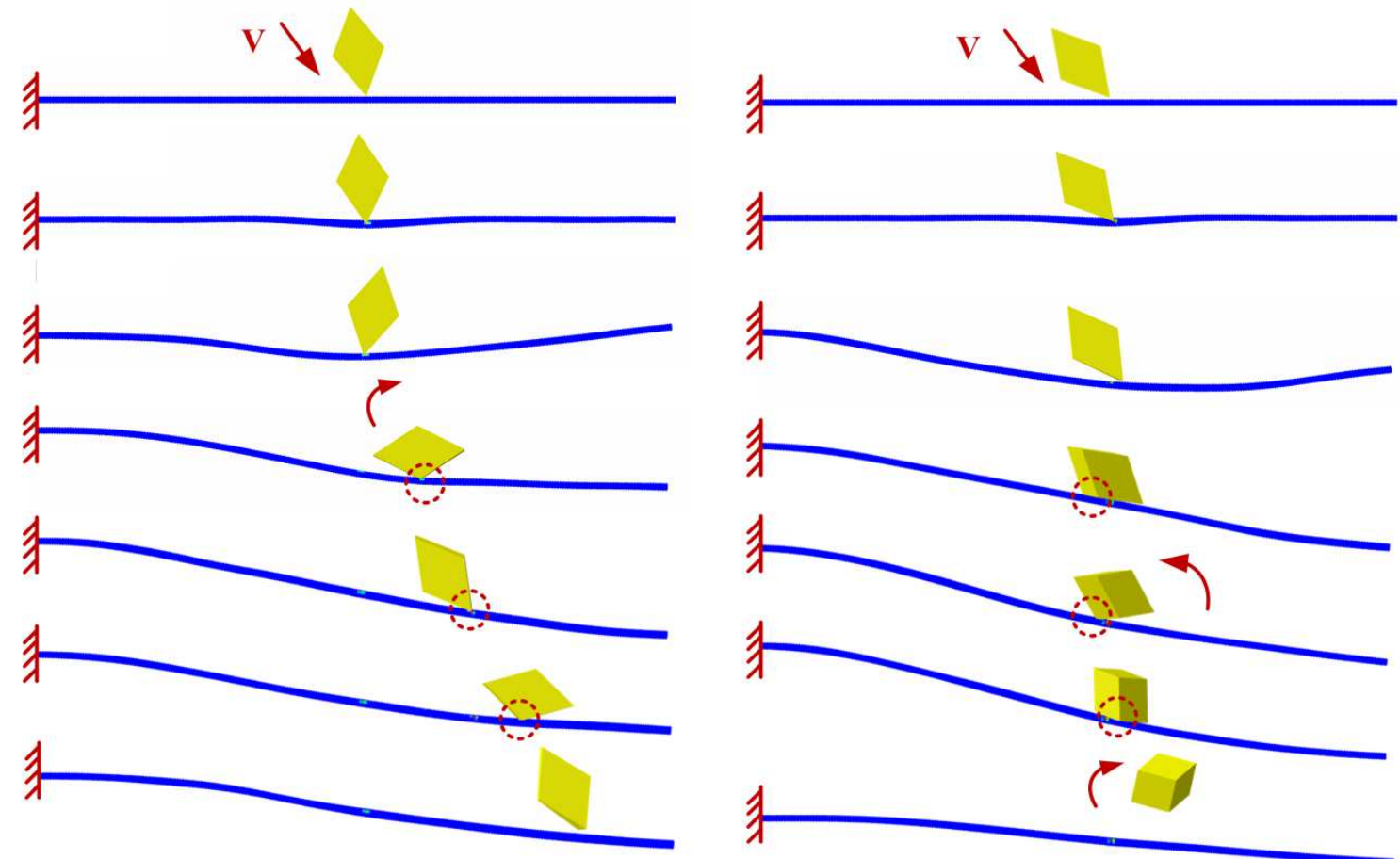

(a) case $1: V=20 \mathrm{~m} / \mathrm{s}, \alpha_{i}=60^{\circ}, \theta_{i}=10^{\circ}$

(b) case2: $V=20 \mathrm{~m} / \mathrm{s}, \alpha_{i}=60^{\circ}, \theta_{i}=40^{\circ}$
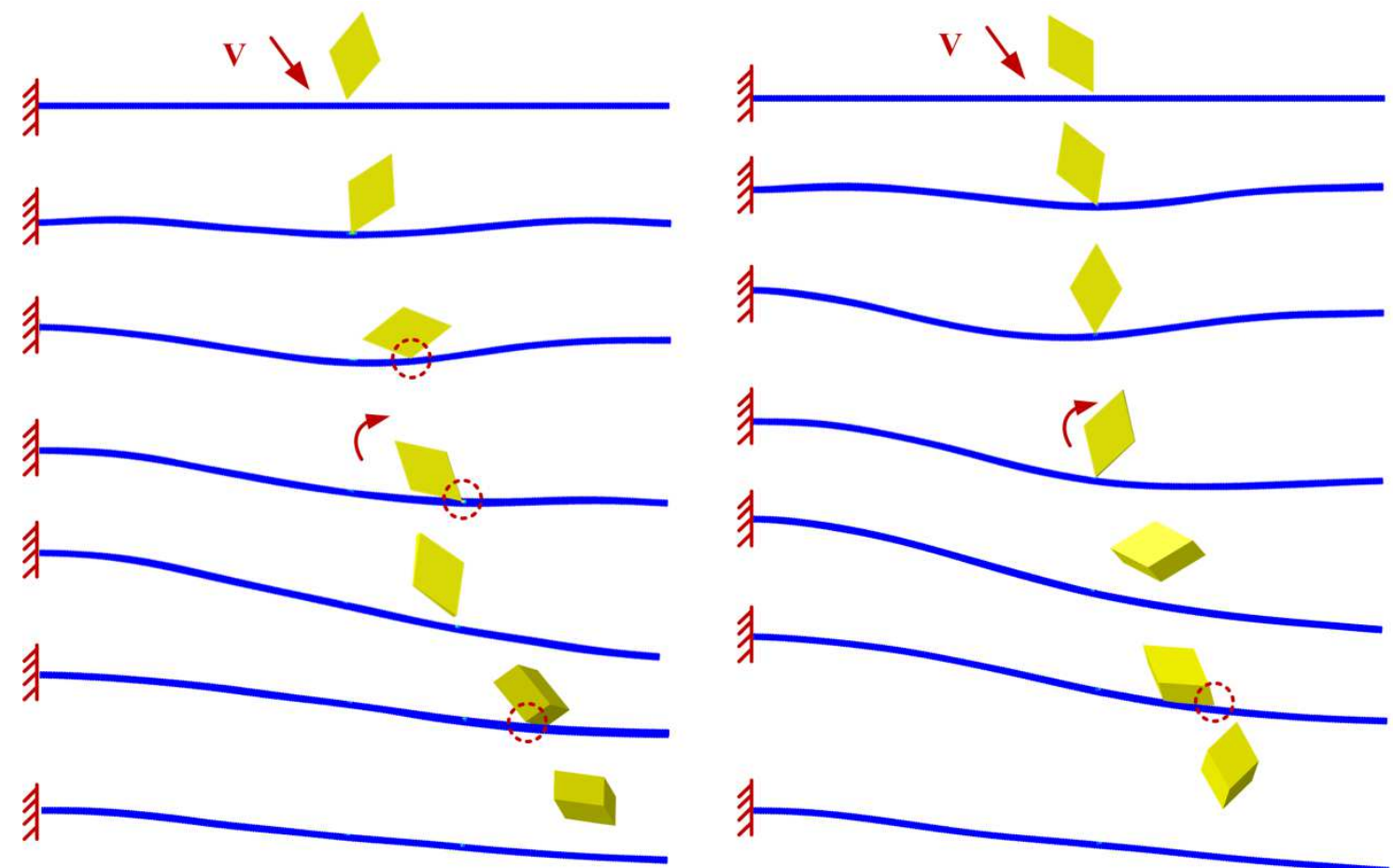

(c) case3: $V=20 \mathrm{~m} / \mathrm{s}, \alpha_{i}=60^{\circ}, \theta_{i}=-10^{\circ}$

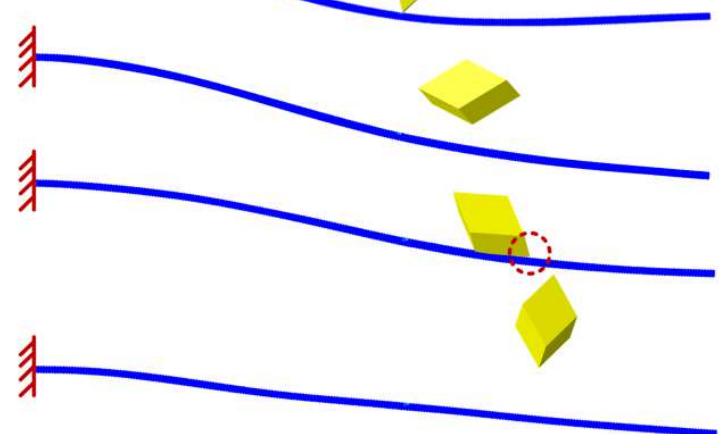

(d) case 4: $V=20 \mathrm{~m} / \mathrm{s}, \alpha_{i}=60^{\circ}, \theta_{i}=30^{\circ}$

Fig.25. Influence of impact angle $\alpha_{i}$ and orientation angle $\theta_{i}$ on particle motion behavior when impact cantilever beam 


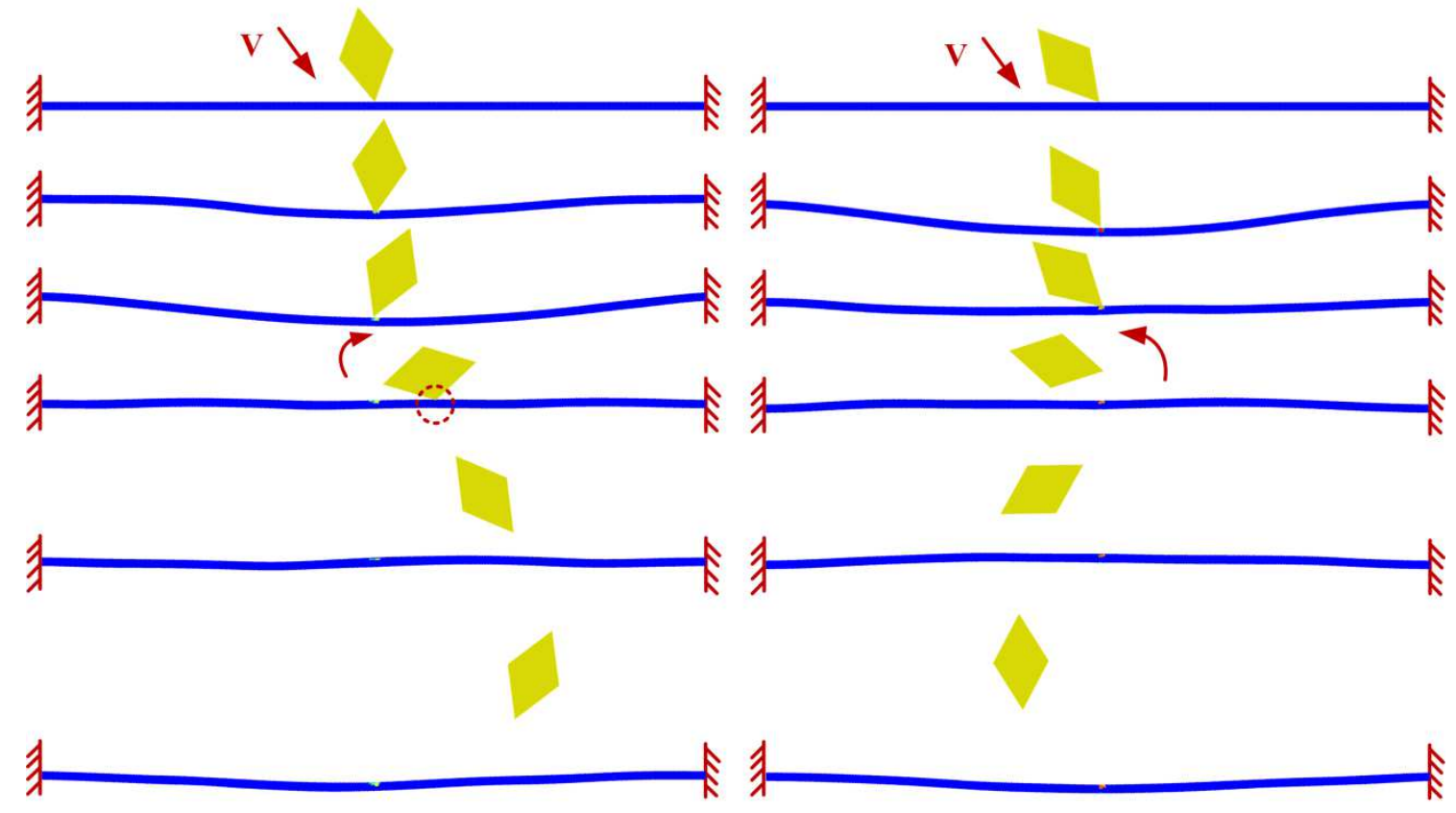

(a) case $1: V=20 \mathrm{~m} / \mathrm{s} \alpha_{i}=60^{\circ} \theta_{i}=10^{\circ}$

(b) case $2: V=20 \mathrm{~m} / \mathrm{s} \alpha_{i}=60^{\circ} \theta_{i}=40^{\circ}$

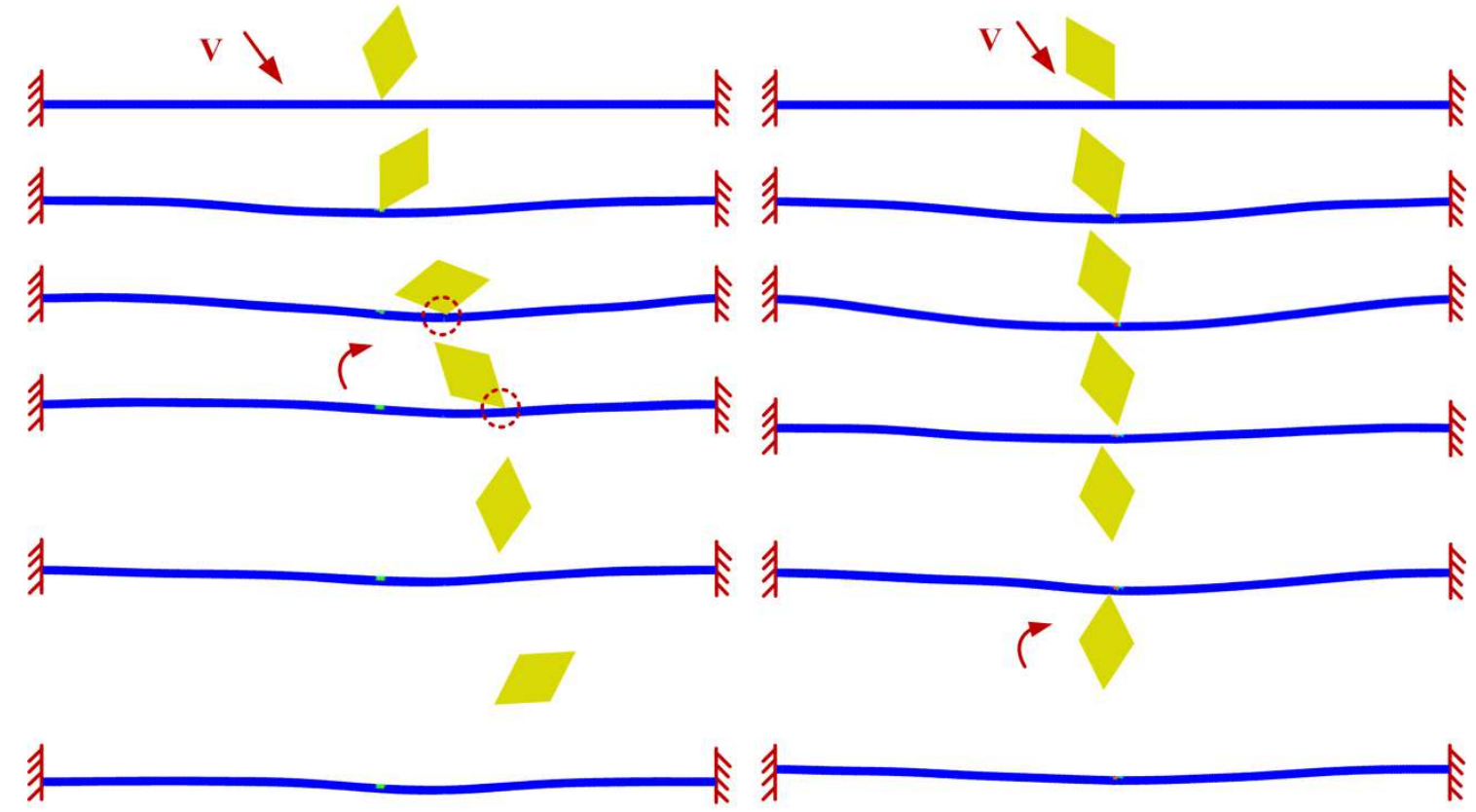

(c) case $3: V=20 \mathrm{~m} / \mathrm{s}, \alpha_{i}=60^{\circ}, \theta_{i}=-10^{\circ}$

(d) case4: $V=20 \mathrm{~m} / \mathrm{s}, \alpha_{i}=60^{\circ}, \theta_{i}=30^{\circ}$

Fig.26. Influence of impact angle $\alpha_{i}$ and orientation angle $\theta_{i}$ on particle motion behavior when impact fixed-fixed beam 


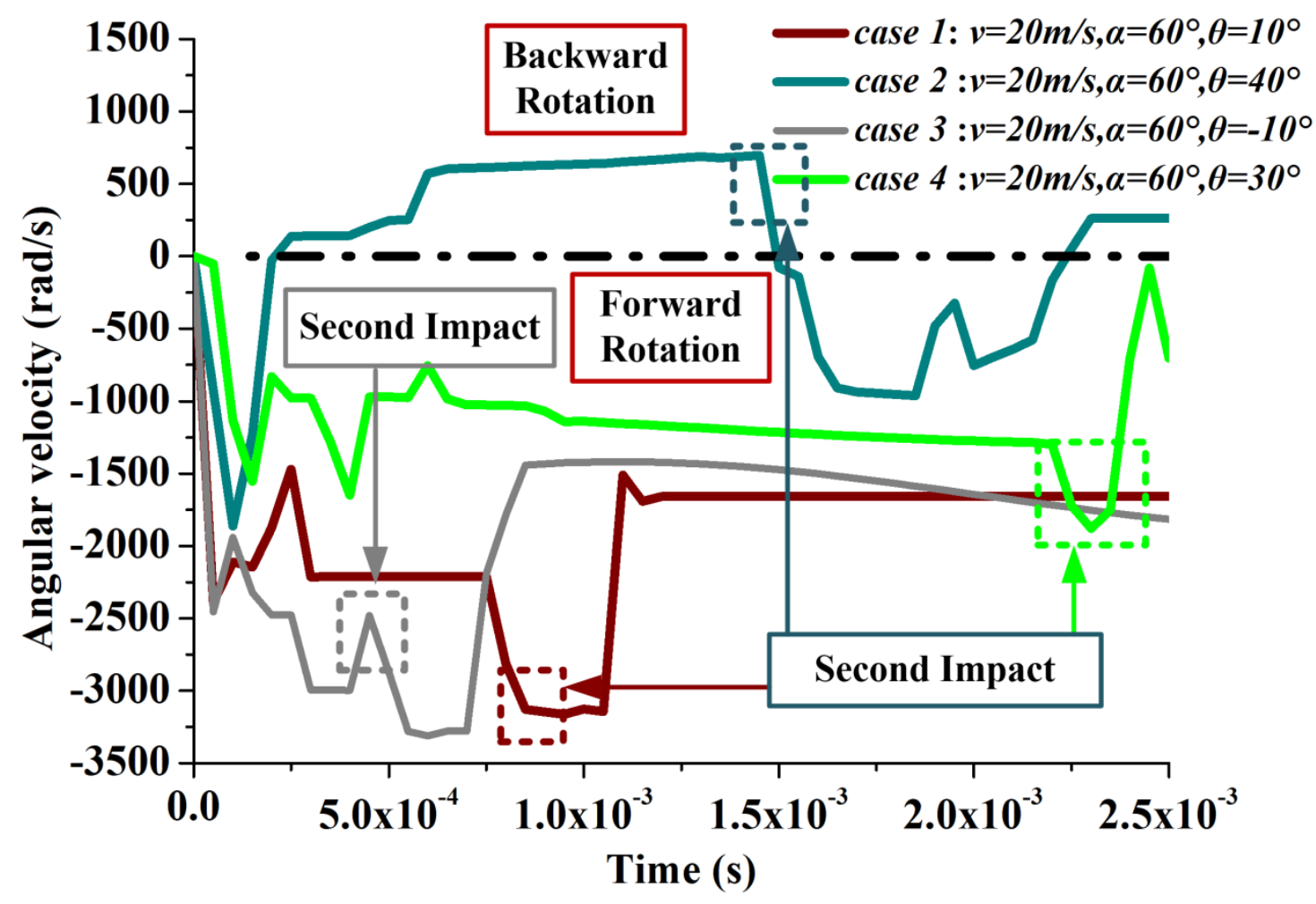

(a) Rhomboid-shaped particle impact cantilever beam

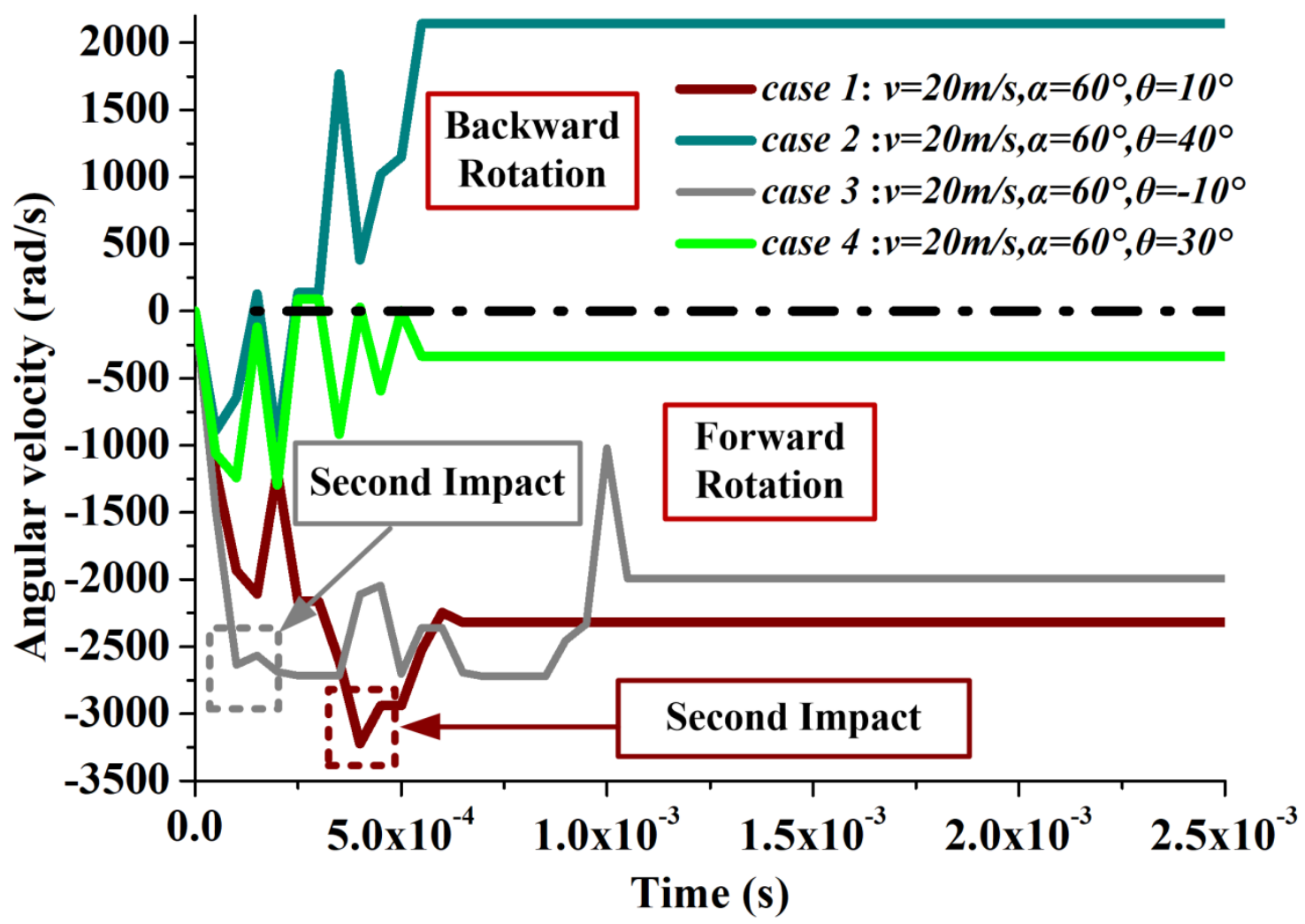

(b) Rhomboid-shaped particle impact fixed-fixed beam

Fig.27. History curves of particle angular velocity with time under four impact conditions 
(a) case $1: \mathrm{v}=20 \mathrm{~m} / \mathrm{s} \alpha_{i}=60^{\circ} \theta_{i}=10^{\circ}$

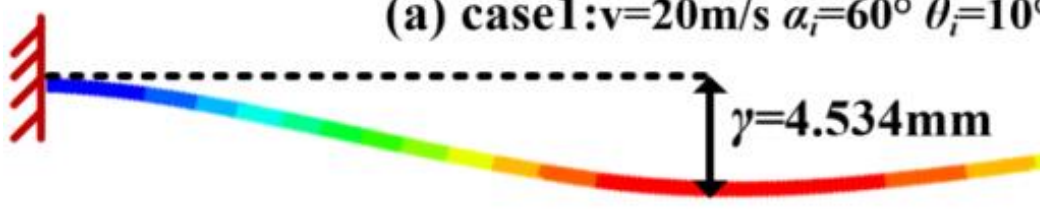

(b) case $2: \mathrm{v}=20 \mathrm{~m} / \mathrm{s} \alpha_{i}=60^{\circ} \theta_{i}=40^{\circ}$

(c) case $3: \mathrm{v}=20 \mathrm{~m} / \mathrm{s} \alpha_{i}=60^{\circ} \theta_{i}=-10^{\circ}$

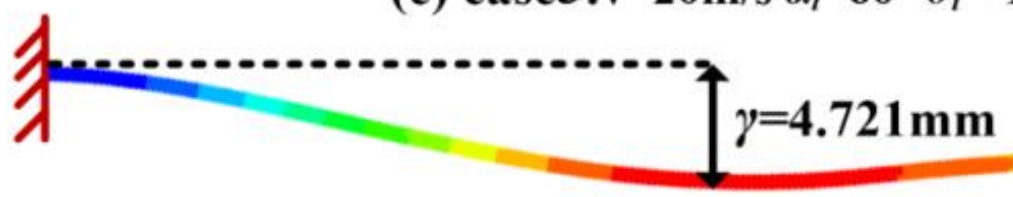

(d) case $4: \mathrm{v}=20 \mathrm{~m} / \mathrm{s} \quad \alpha_{i}=60^{\circ} \theta_{i}=30^{\circ}$

(a) Rhomboid-shaped particle impact cantilever beam

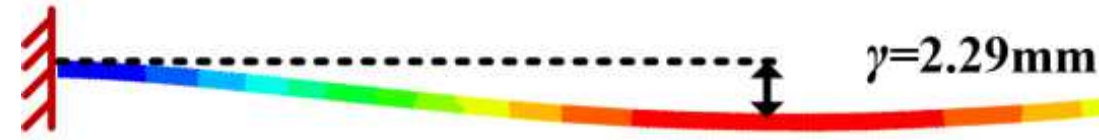

(a) case $1: v=20 \mathrm{~m} / \mathrm{s} \alpha_{i}=60^{\circ} \theta_{i}=10^{\circ}$

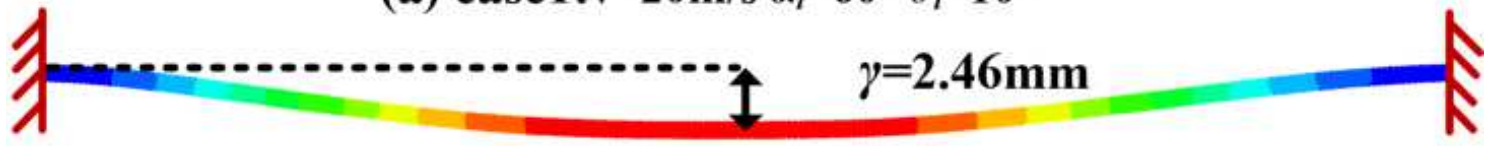

(b) case $2: \mathrm{v}=20 \mathrm{~m} / \mathrm{s} \alpha_{i}=60^{\circ} \theta_{i}=40^{\circ}$

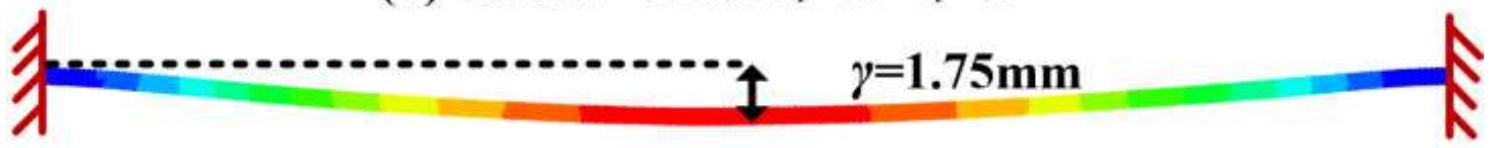

(c) case $3: \mathrm{v}=20 \mathrm{~m} / \mathrm{s} \alpha_{i}=60^{\circ} \theta_{i}=-10^{\circ}$

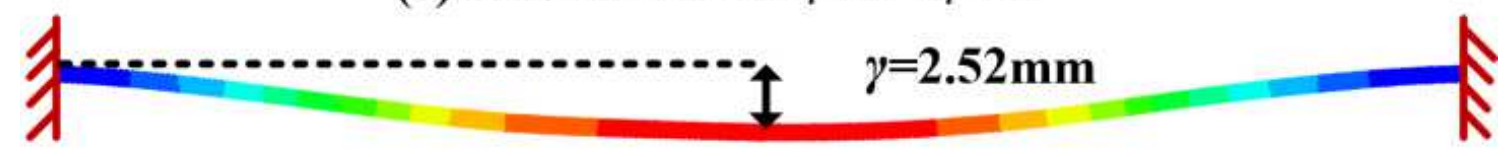

(d) case $4: \mathrm{v}=20 \mathrm{~m} / \mathrm{s} \alpha_{i}=60^{\circ} \quad \theta_{i}=30^{\circ}$

(b) Rhomboid-shaped particle impact fixed-fixed beam

Fig.28. Maximum deflection $\gamma$ at the impact point caused by rhomboid-shaped particle under four impact conditions 


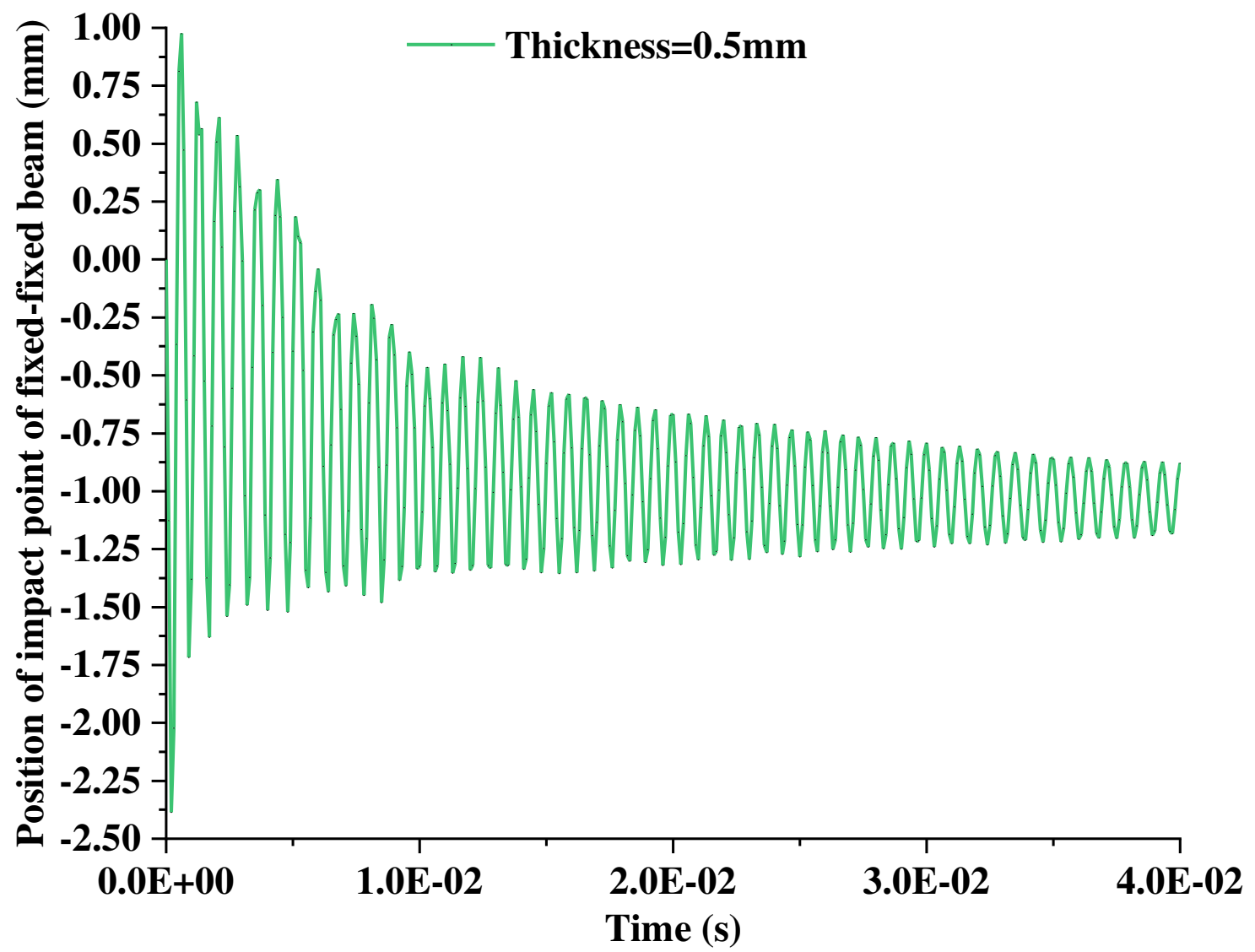

(a) Fixed-fixed beam: thickness $\mathrm{h}=0.5 \mathrm{~mm}$

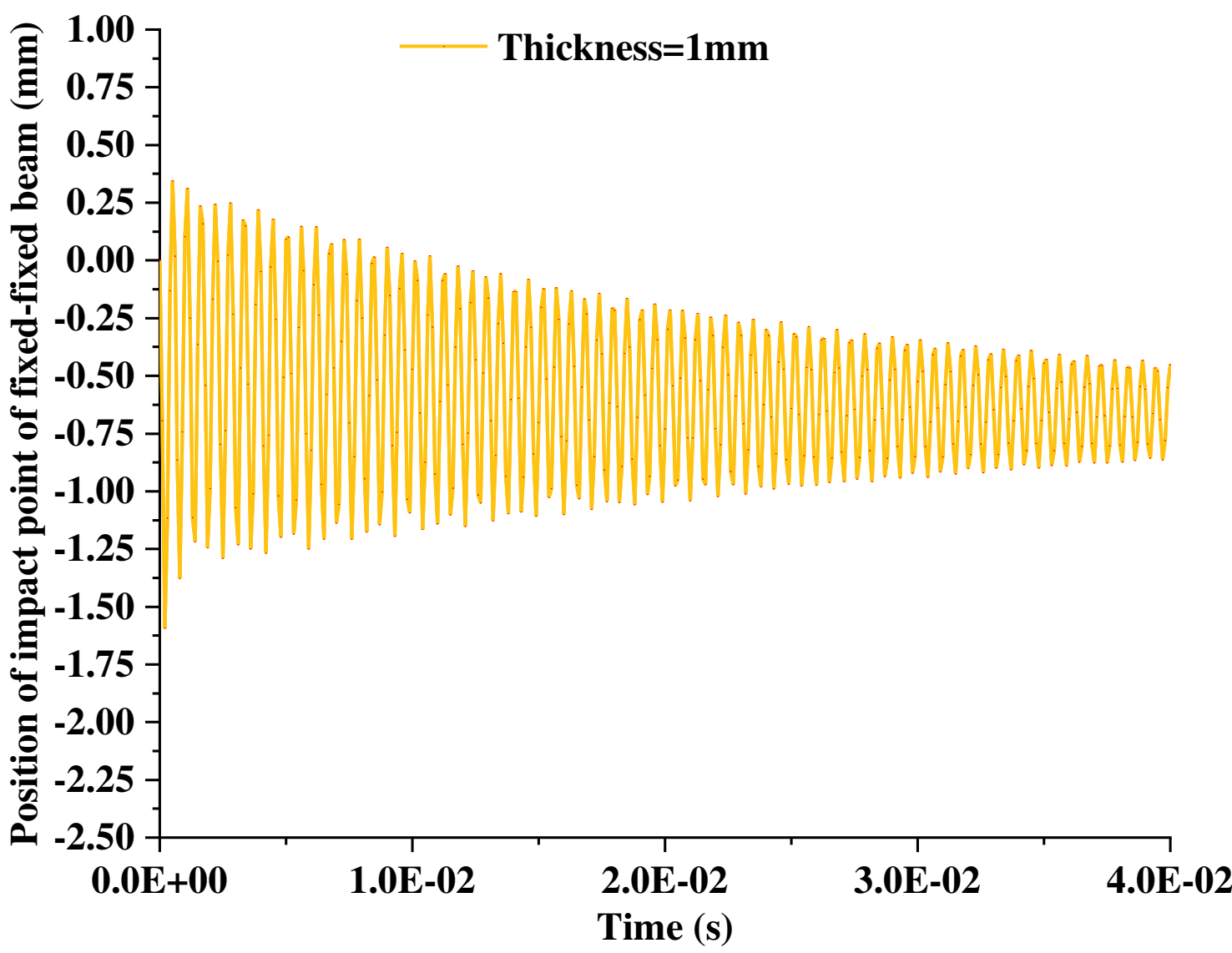

(b) Fixed-fixed beam: thickness $\mathrm{h}=1 \mathrm{~mm}$ 


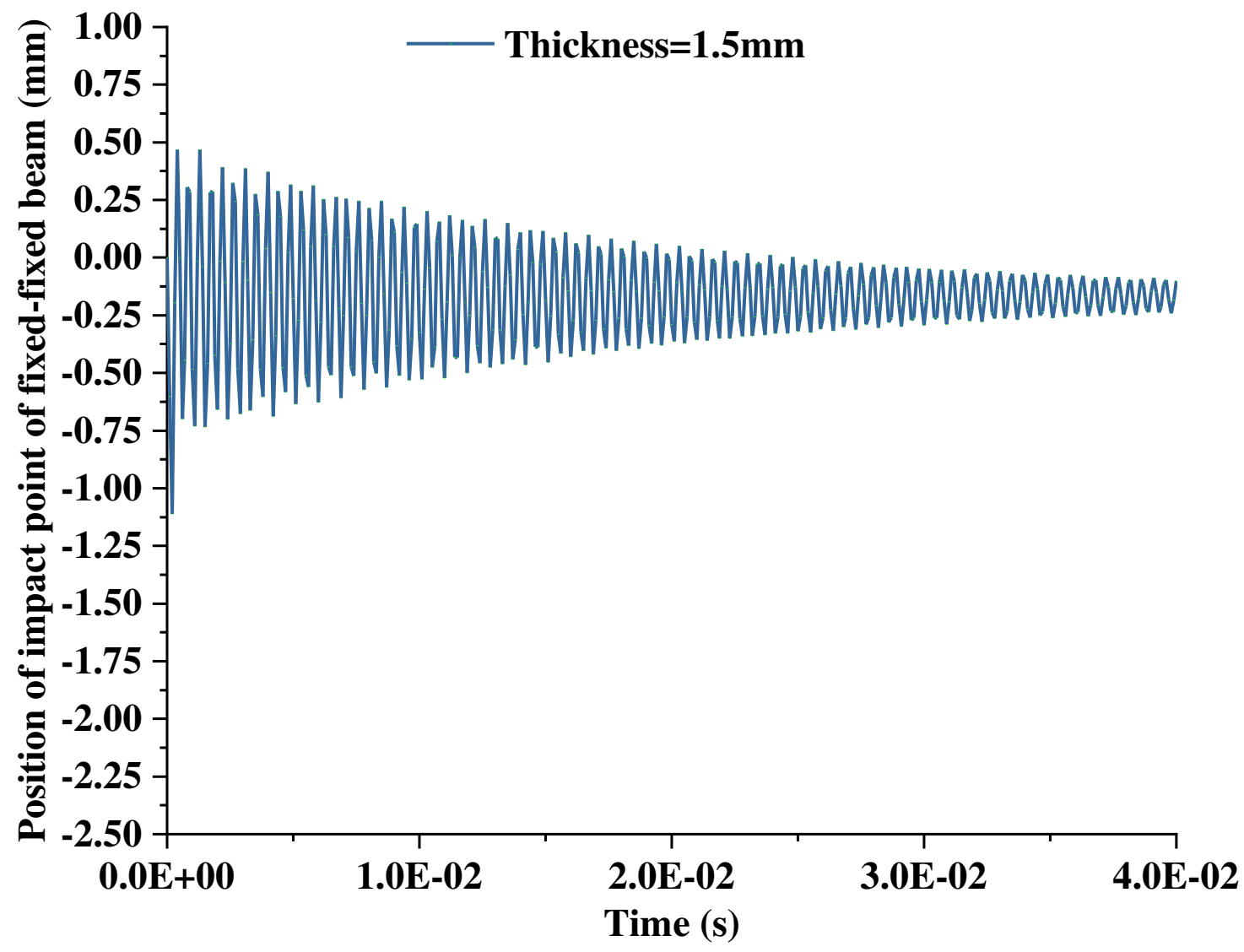

(c) Fixed-fixed beam: thickness $\mathrm{h}=1.5 \mathrm{~mm}$

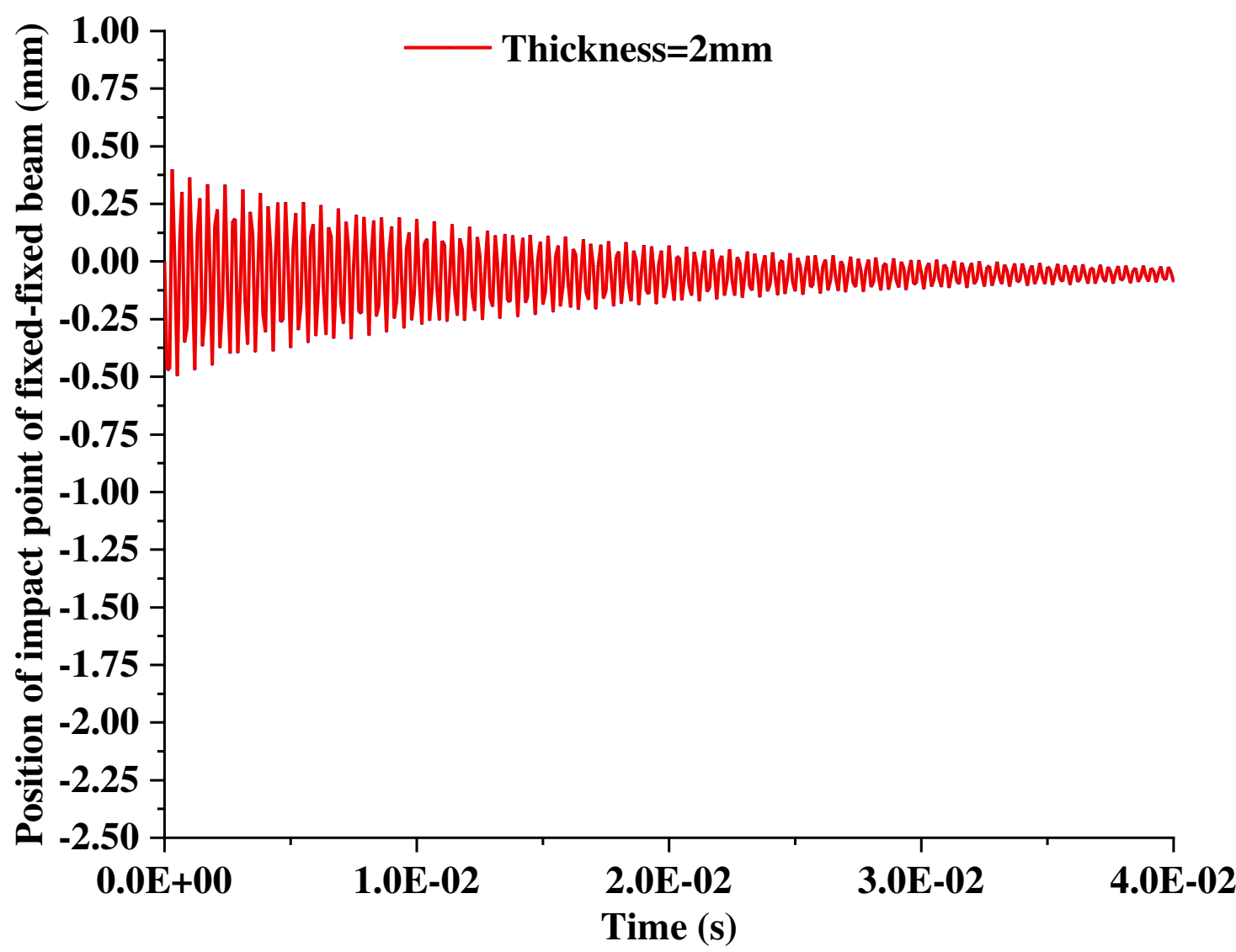

(d) Fixed-fixed beam: thickness $\mathrm{h}=2 \mathrm{~mm}$

Fig.29. Variation of amplitude with time of fixed-fixed beams with different thickness after impact 
Figures

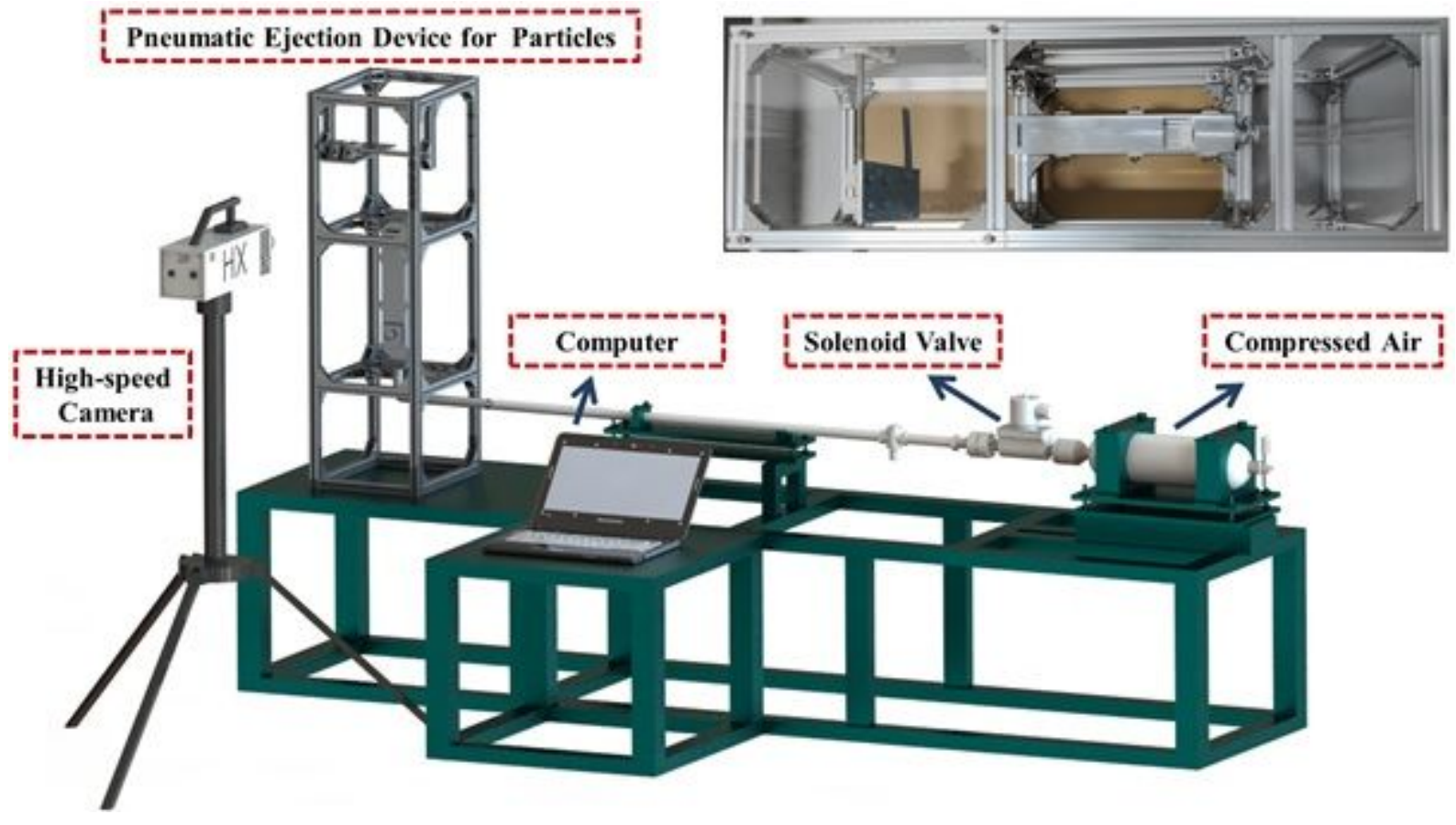

Figure 1

Pneumatic Ejection and Imaging System for Solid Particles

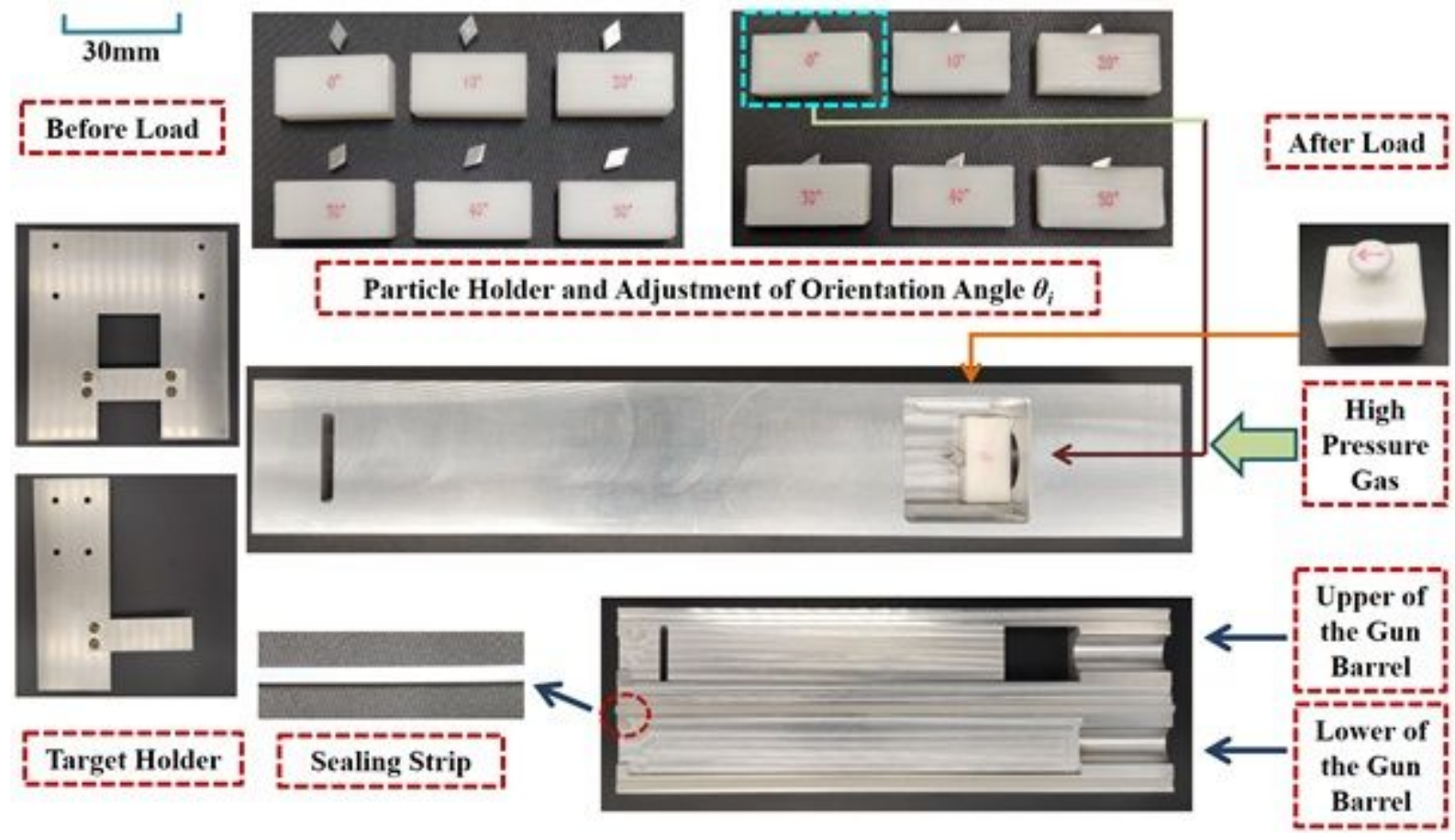

Figure 2

Adjustment of particle orientation angle $\theta i$ and exhibition of variable cross-section gun barrel 


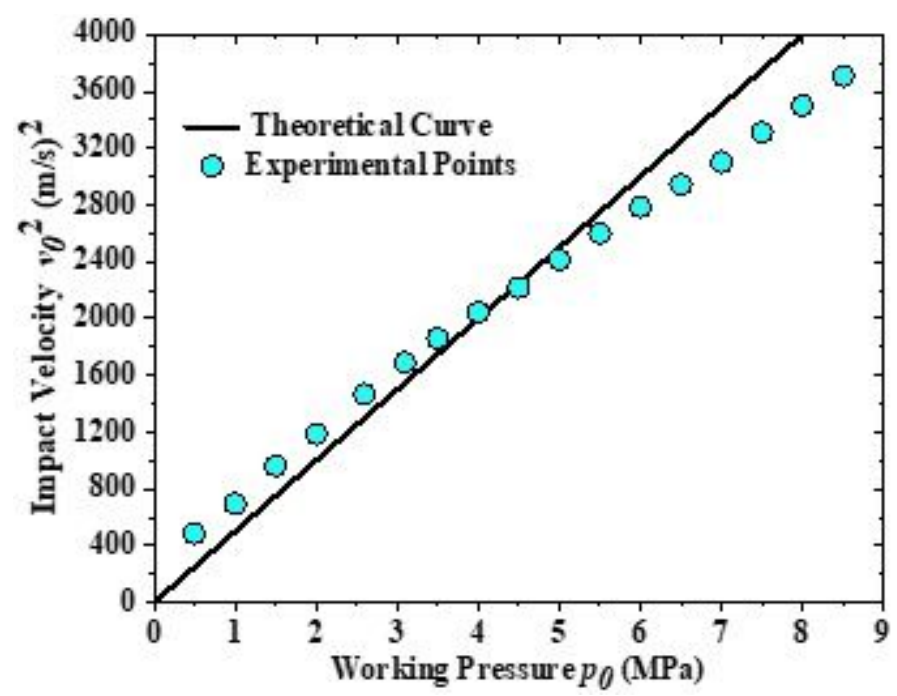

Figure 3

The relationship between initial launch velocity v02 of gun barrel and working pressure p0
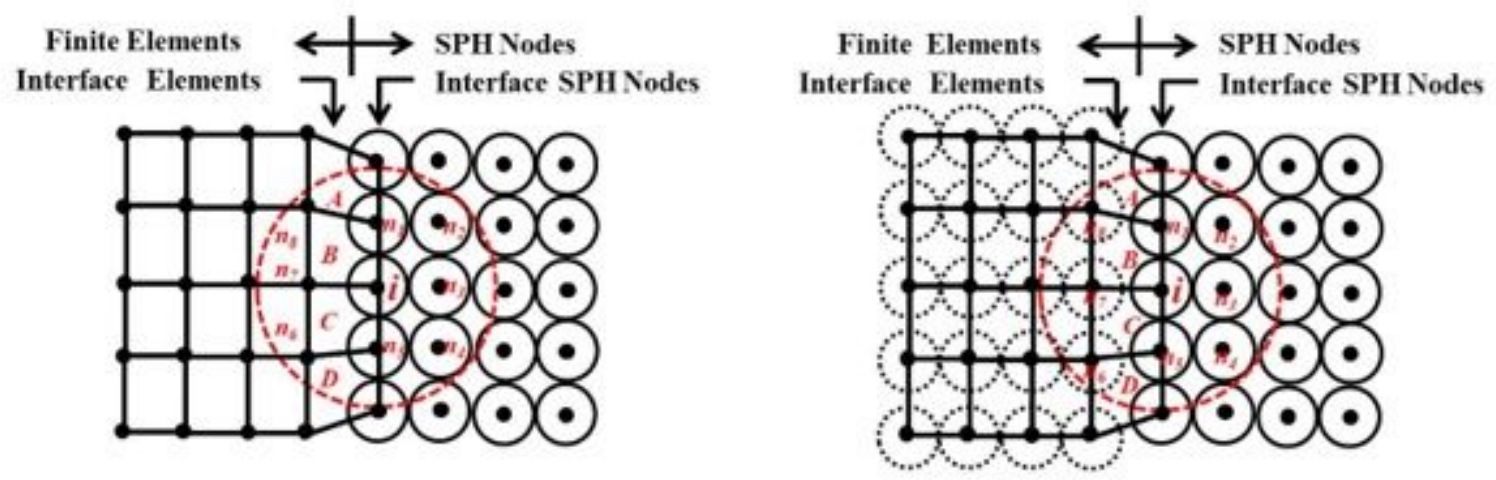

(a) SPH nodes attachment to grid without background particles [24] (b) SPH nodes attachment to grid with background particles [25]

Figure 4

SPH particles attached to finite elements 


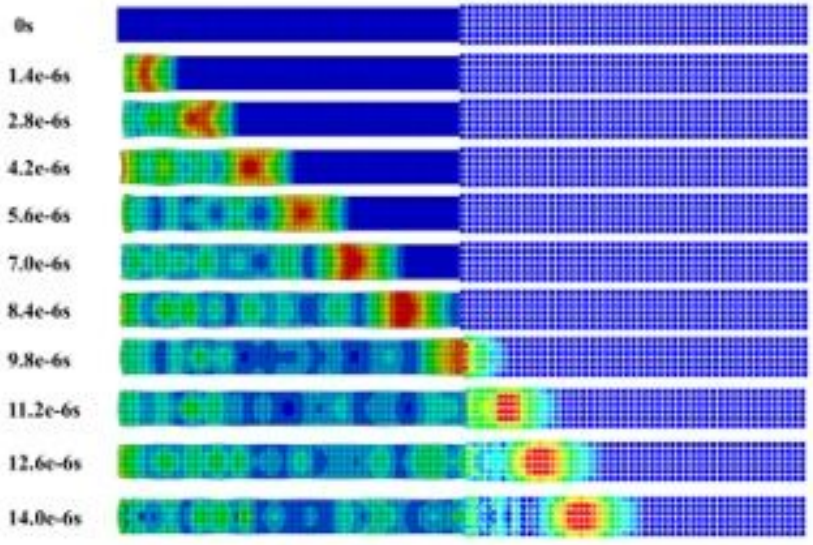

(a) Stress wave propagation of FEM-SPH coupled model

$0 s$
$1.40-6 s$
$2.80-6 s$
$4.2 e-6 s$
$5.6 e-6 s$
$7.00-6 s$
$8.40-6 s$
$9.80-6 s$
$11.2 e-6 s$
$12.6 s-6 s$
$14.00-6 s$

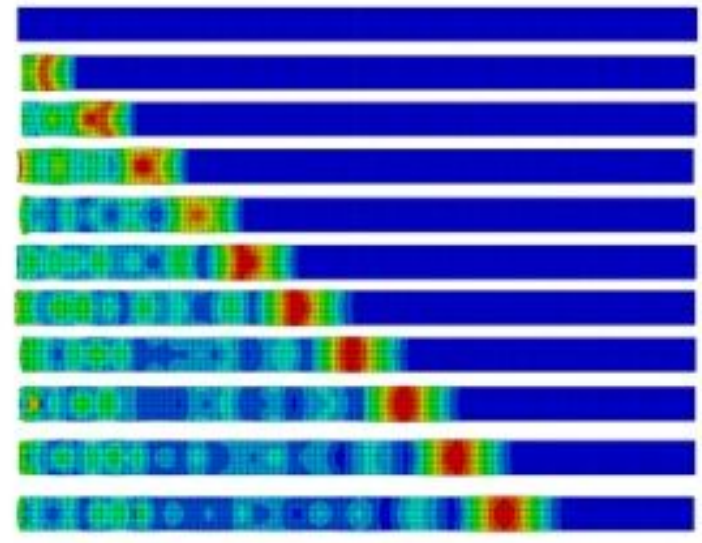

(b) Stress wave propagation of FEM model

\section{Figure 5}

Comparison of stress wave propagation between FEM-SPH model and FEM model

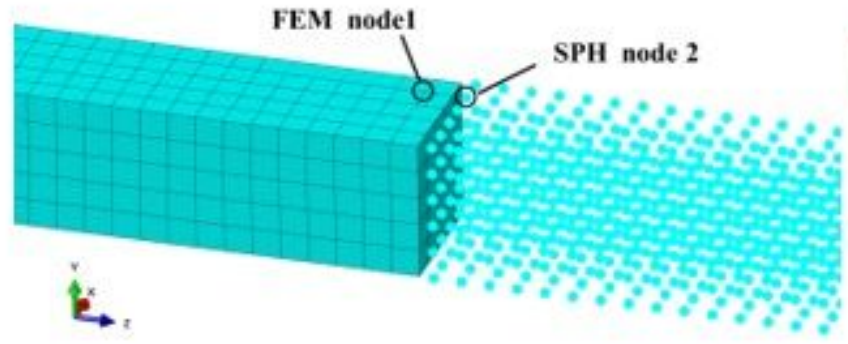

(a) Reference points on FEM-SPH coupled model

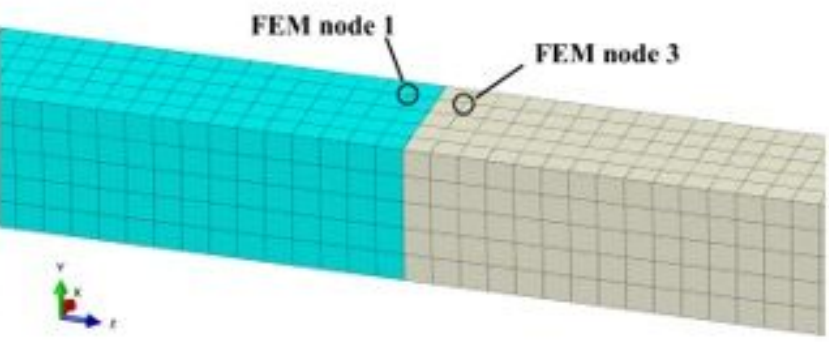

(b) Reference points on FEM model

\section{Figure 6}

Labeling of reference finite element nodes and SPH particles 


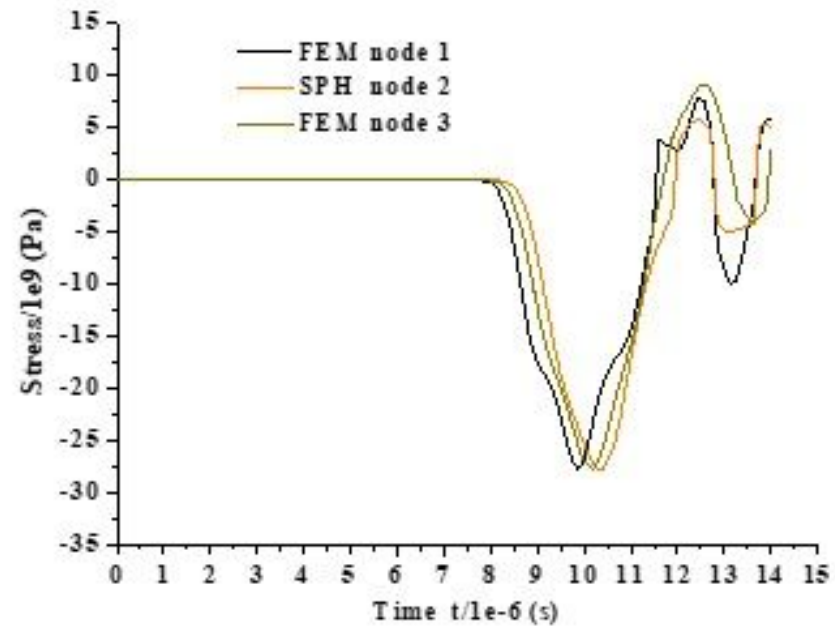

(a) The relationship of stress change with time

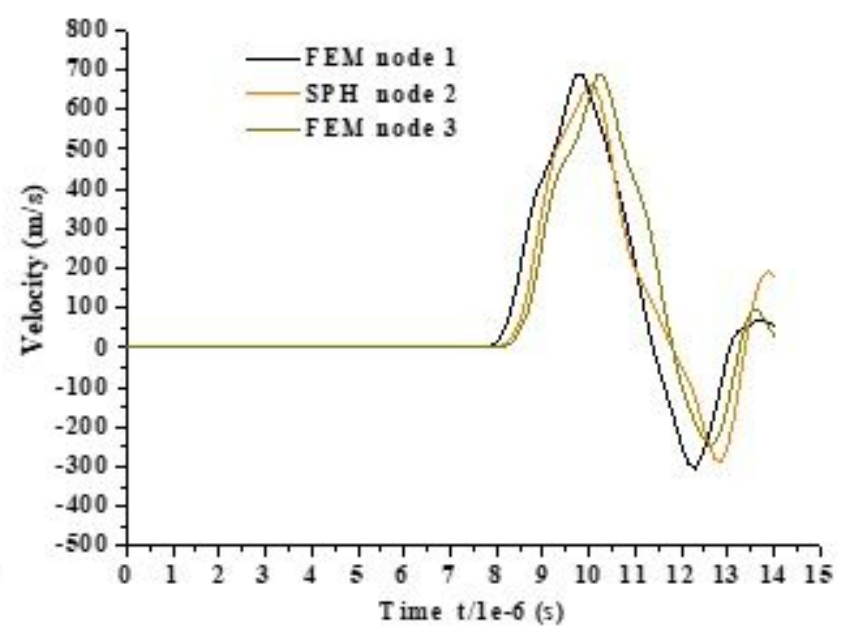

(b) The relationship of velocity change with time

Figure 7

Field variables of three reference points change with time

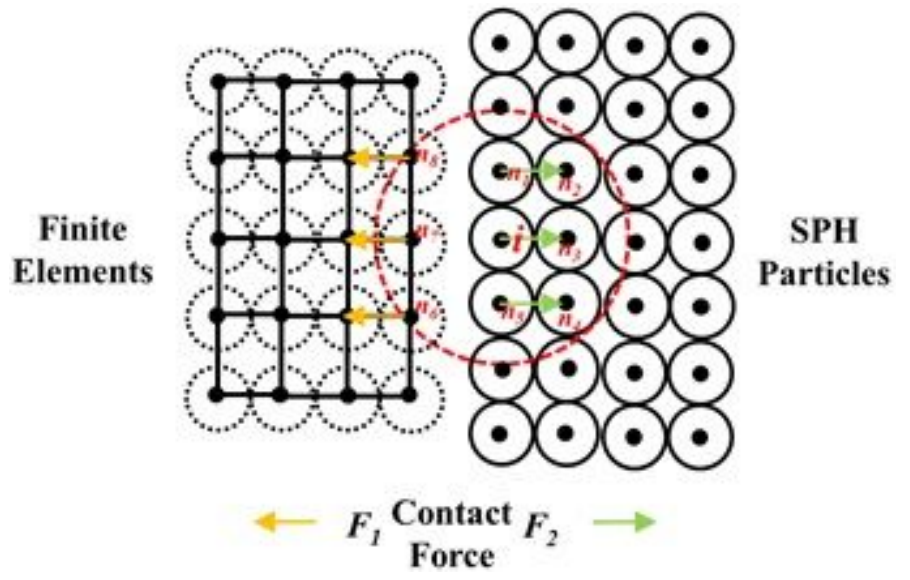

Figure 8

SPH particles contact with finite elements 


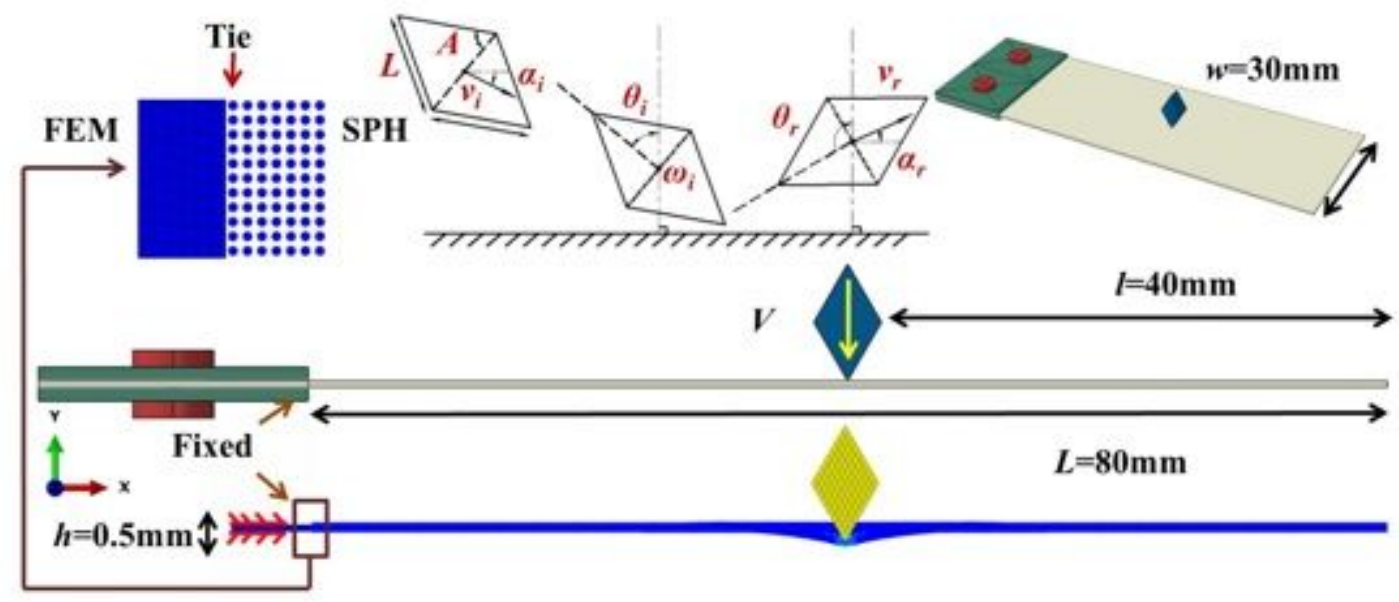

(a) Rhomboid shaped particle impacting cantilever beam

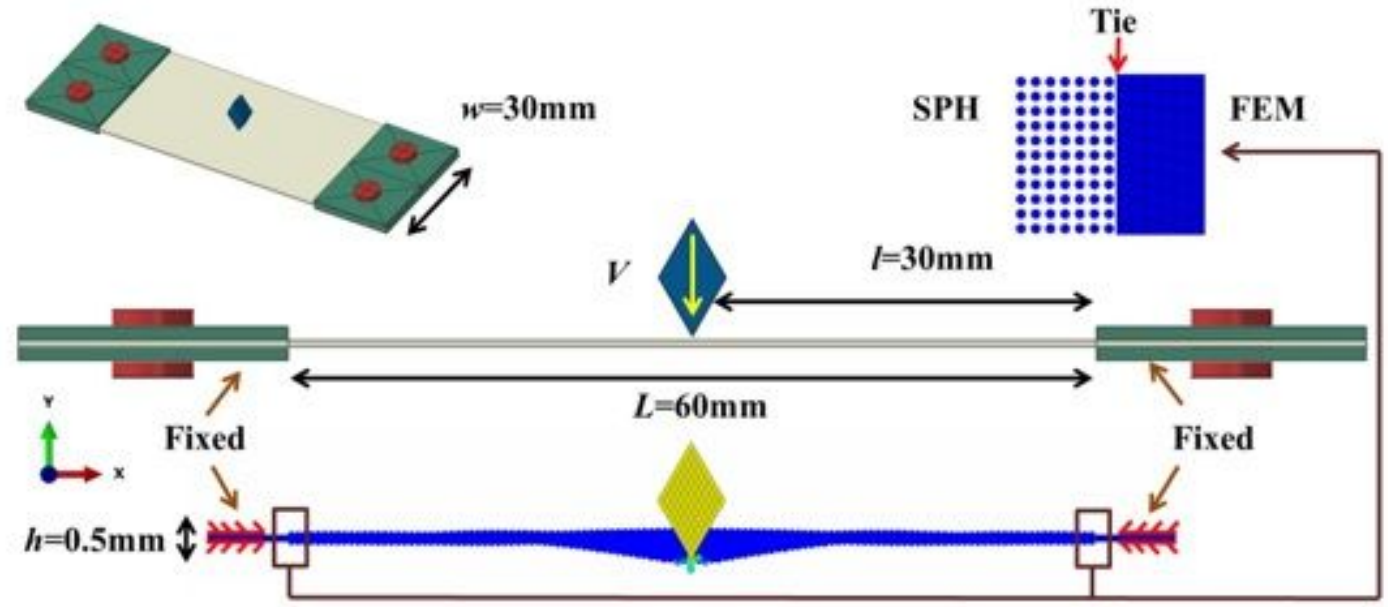

(b) Rhomboid shaped particle impacting fixed-fixed beam

\section{Figure 9}

Coupled FEM-SPH numerical model of rhomboid-shaped particle impacts Al6061-T6 thin sheet 

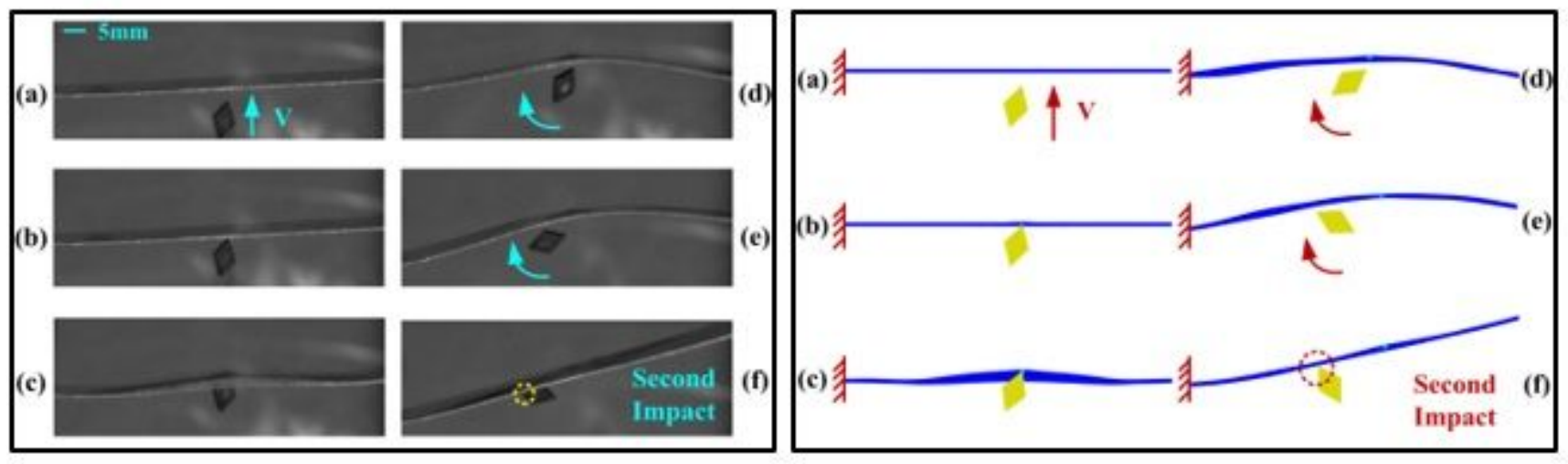

(a) Rhomboid-shaped particle impacting on cantilever beam: $V=36.75 \mathrm{~m} / \mathrm{s}, \alpha_{i}=90^{\circ}, \theta_{i}=-16^{\circ}$
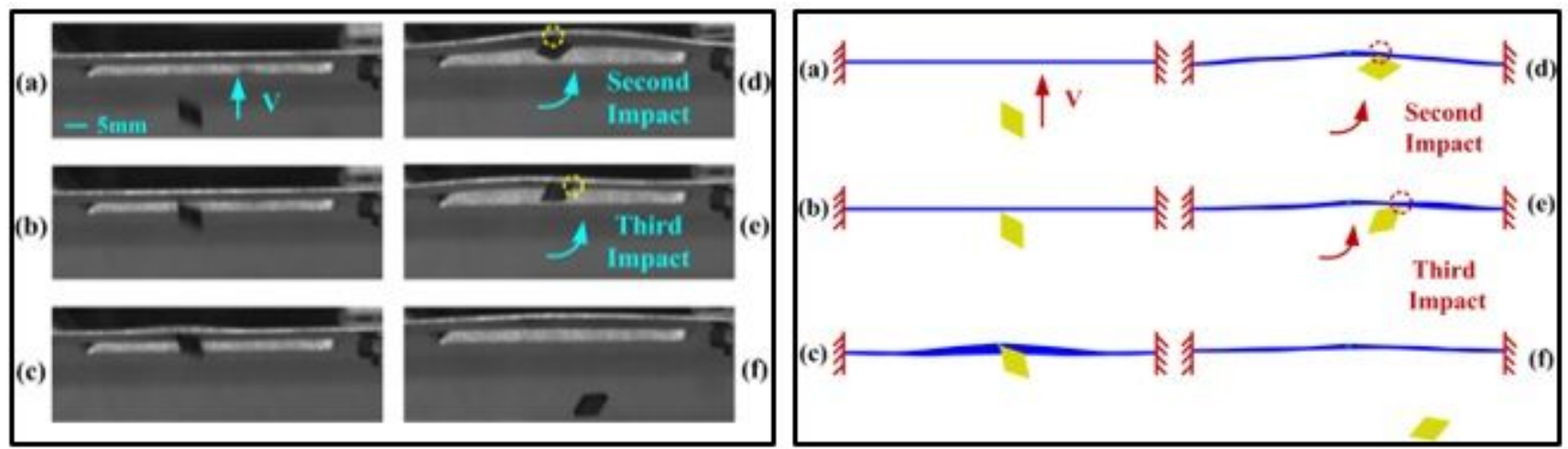

(b) Rhomboid-shaped particle impacting on fixed-fixed-beam: $\mathrm{V}=34.75 \mathrm{~m} / \mathrm{s}, \alpha_{\mathrm{l}}=90^{\circ}, \theta_{\mathrm{i}}=31^{\circ}$

\section{Figure 10}

Comparison of experimental and simulated results of rhomboid-shaped particle impacting on Al6061-T6 thin plate 

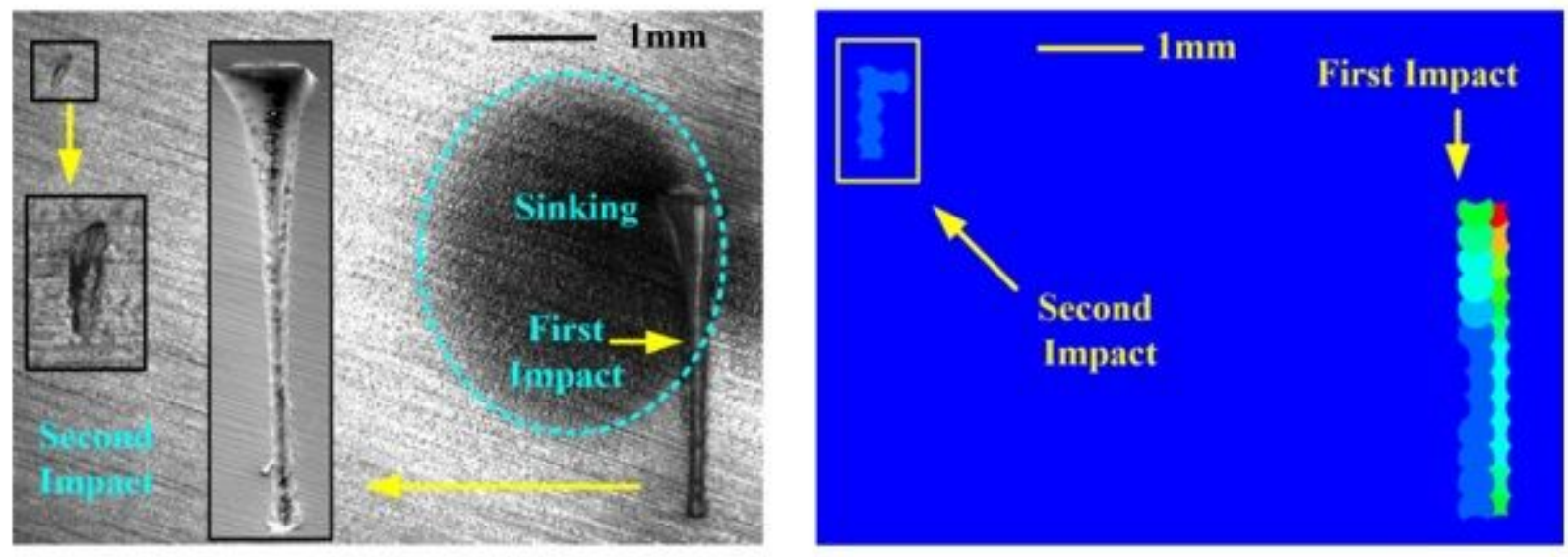

(a) Contour of craters on cantilever beam formed by rhomboid-shaped particle
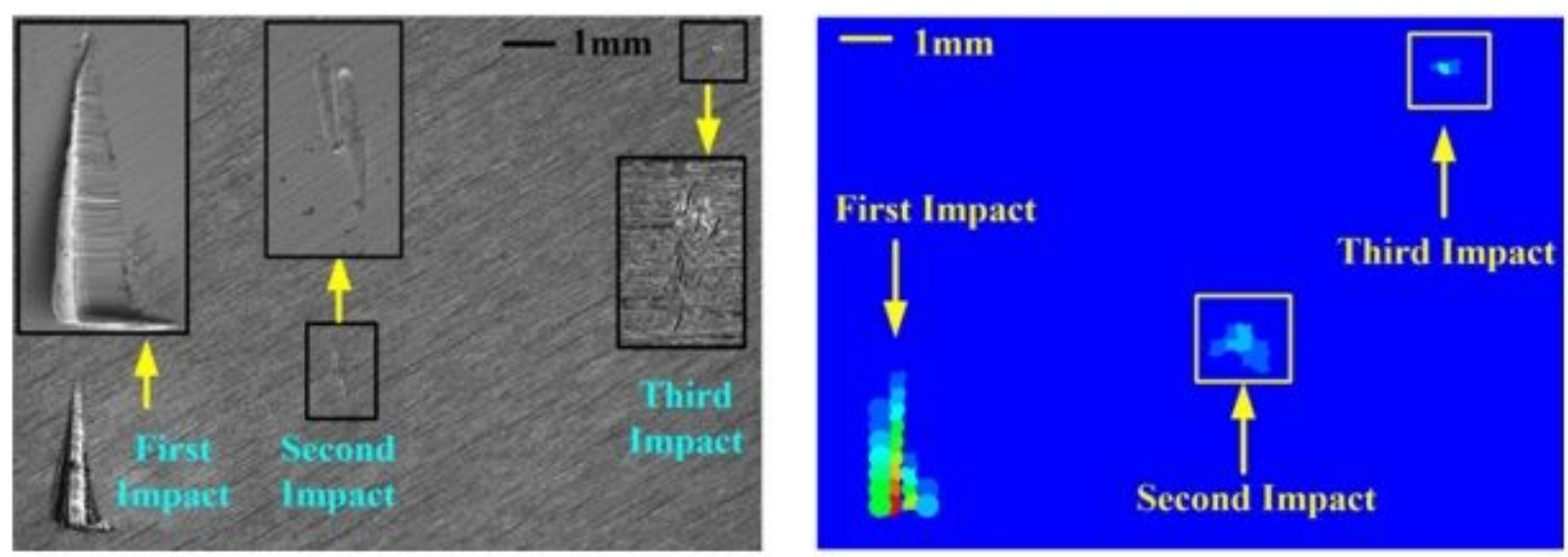

(b) Contour of craters on fixed-fixed beam formed by rhomboid-shaped particle

\section{Figure 11}

Comparison of experimental and simulated results of craters profile formed by rhomboid-shaped particle
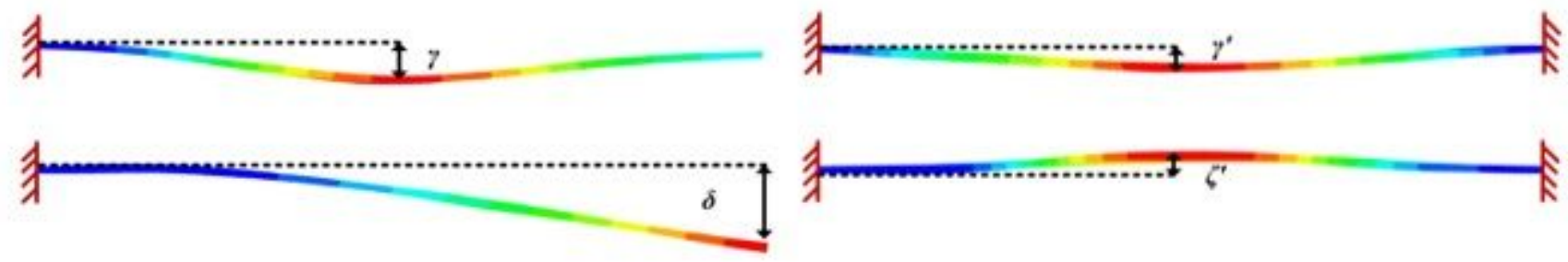

(a) Deformation deflection diagram of cantilever beam

(b) Deformation deflection diagram of fixed-fixed-beam

\section{Figure 12}

Deflection diagram of cantilever beam and fixed-fixed beam after being impacted by particles $(\mathrm{V}=20 \mathrm{~m} / \mathrm{s}$, ai $=90^{\circ}, \theta i=0^{\circ}$ ) 


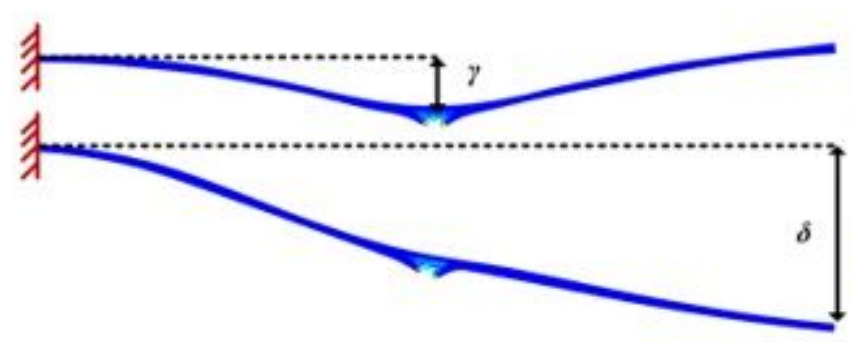

(a) Plastic bending and breakthrough of cantilever beam

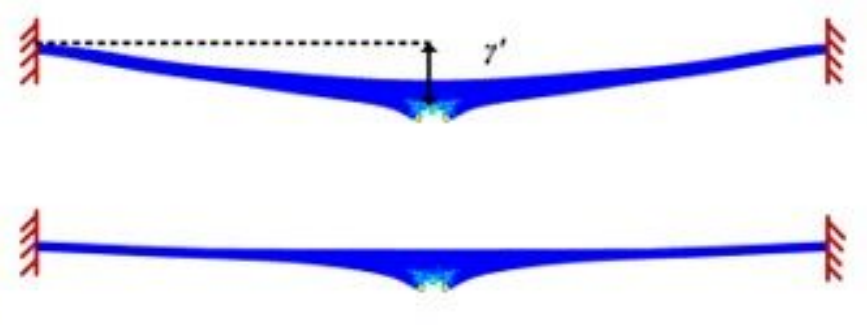

(b) Plastic bending and brealthrough of fixed-fixed beam

\section{Figure 13}

Plastic bending and breakthrough of thin plate (cantilever beam \& fixed-fixed beam) after impacting by rhomboid-shaped particle under medium-high velocity $\left(V=60 \mathrm{~m} / \mathrm{s}, \mathrm{ai}=90^{\circ}, \theta i=0^{\circ}\right)$

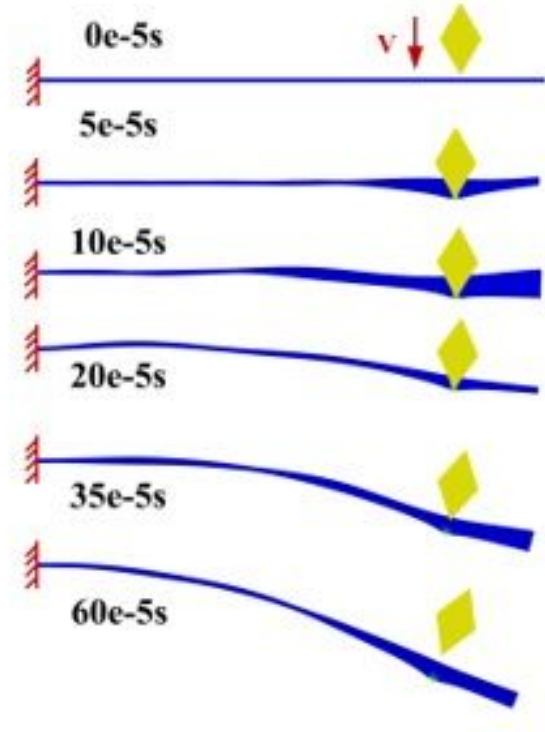

(a) Simulated results of FEM model
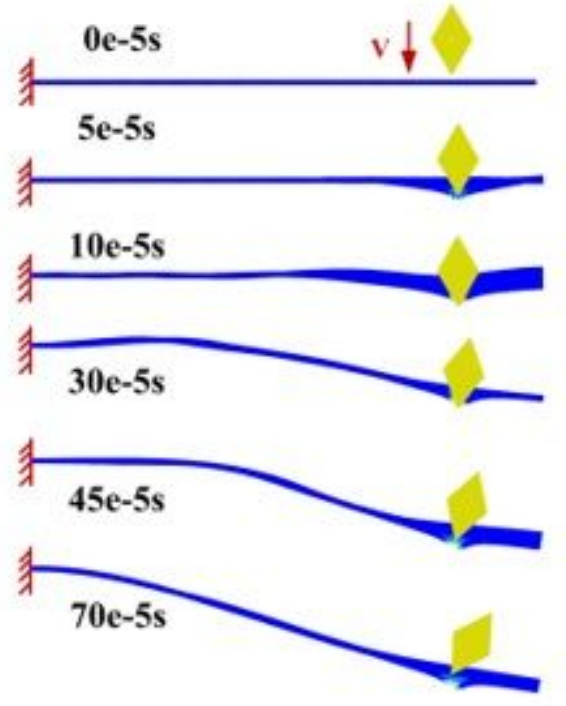

(b) Simulated results of FEM-SPH coupled model

\section{Figure 14}

omparison and verification of different models to capture substrate deformation under medium-high velocity impact conditions $\left(\mathrm{V}=60 \mathrm{~m} / \mathrm{s}, \mathrm{ai}=90^{\circ}, \theta \mathrm{i}=0^{\circ}\right)$
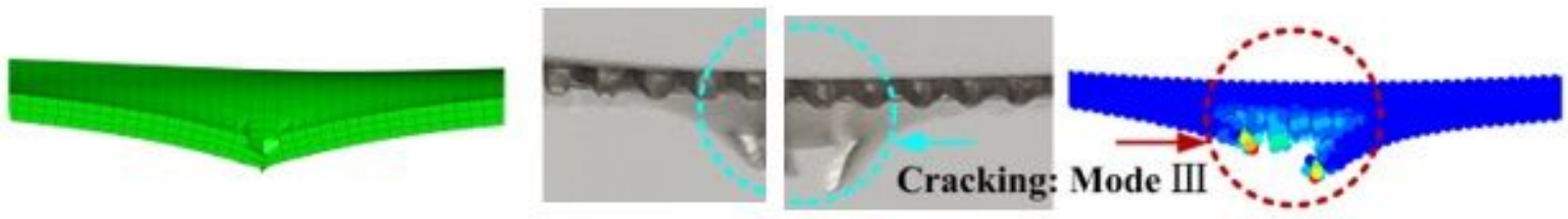
(a) Crater predicted bv FEM model
(b) Crater fror $\mathrm{n}$ experiments
(c) Crater predicted by FEM-SPH coupled model 
Figure 15

Comparison and verification of different models to capture substrate breakdown under medium-high velocity impact conditions

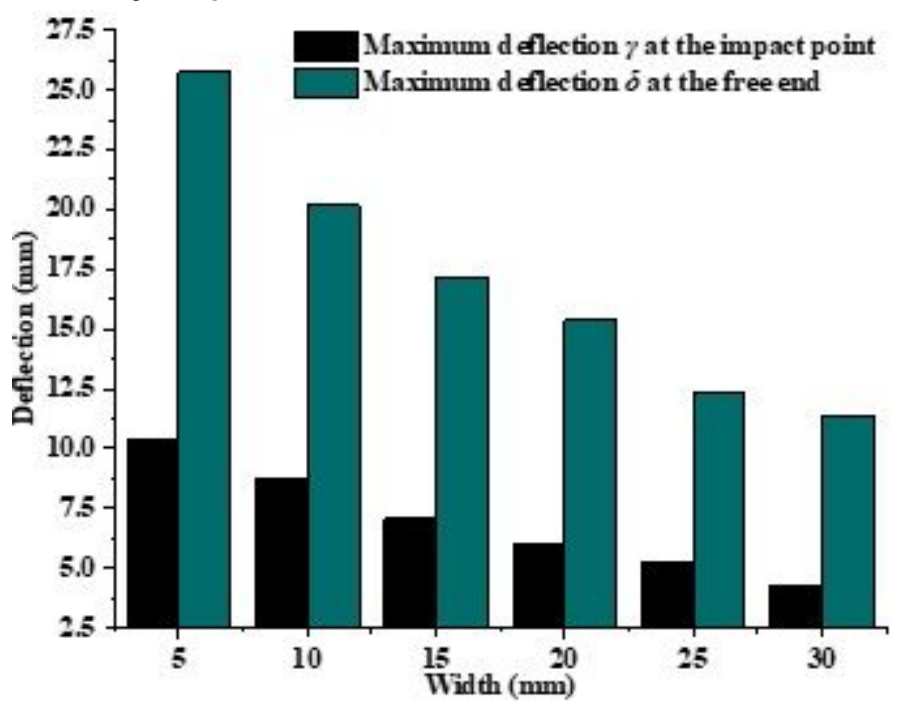

\section{Figure 16}

Variation of maximum deflection $y$ and $\delta$ of cantilever beam with beam width $w\left(V=30 \mathrm{~m} / \mathrm{s}, a i=90^{\circ}, \theta i=0^{\circ}\right.$, $\mathrm{L}=60 \mathrm{~mm}, \mathrm{~h}=0.5 \mathrm{~mm}$ )

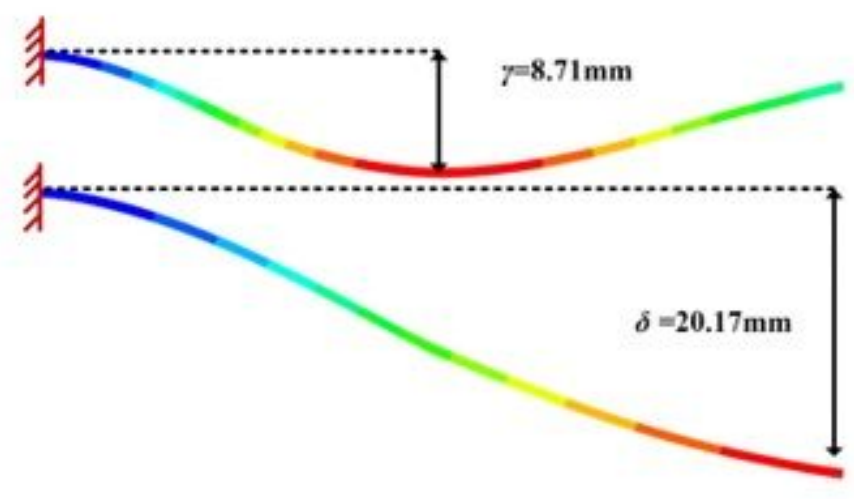

(a) The width of cantilever beam: $w=10 \mathrm{~mm}$

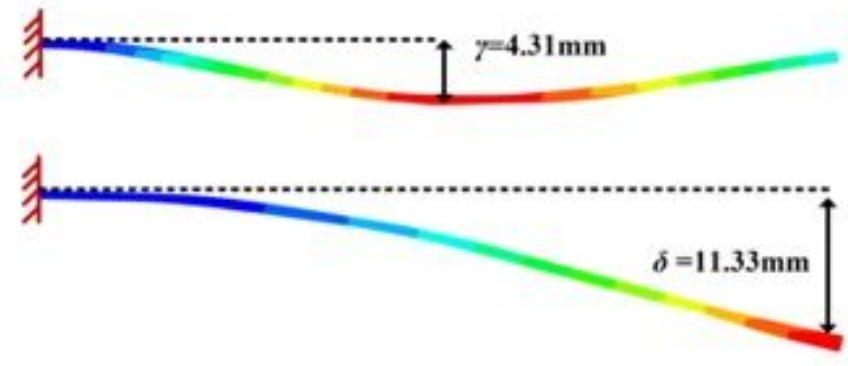

(b) The width of cantilever beam: $w=30 \mathrm{~mm}$

\section{Figure 17}

Deformation behavior of cantilever beams with different widths under the same impact condition $\left(V=30 \mathrm{~m} / \mathrm{s}, \mathrm{ai}=90^{\circ}, \theta \mathrm{i}=0^{\circ}\right)$ 

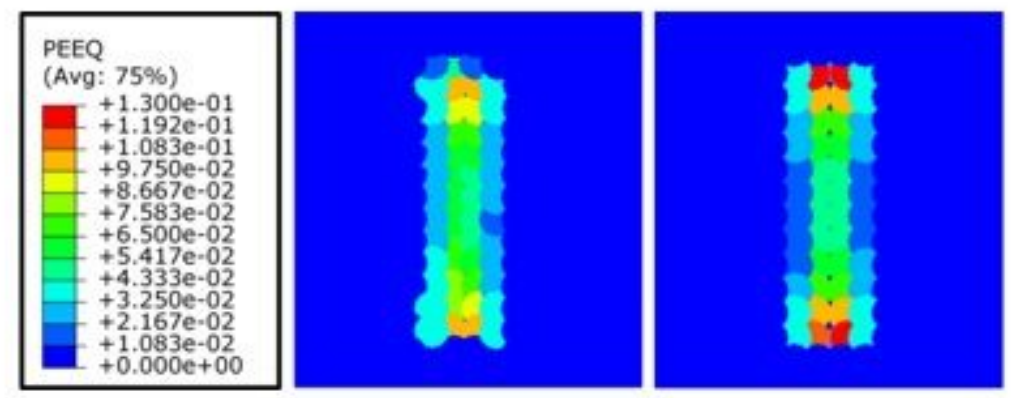

$\begin{array}{ll}\text { (a) The width of cantilever beam: } w=10 \mathrm{~mm} & \text { (b) The width of cantilever beam: } w=30 \mathrm{~mm}\end{array}$

\section{Figure 18}

Crater contour of cantilever beams with different widths under the same impact condition $(\mathrm{V}=30 \mathrm{~m} / \mathrm{s}$, $\left.\mathrm{ai}=90^{\circ} \mathrm{\theta i}=0^{\circ}\right)$
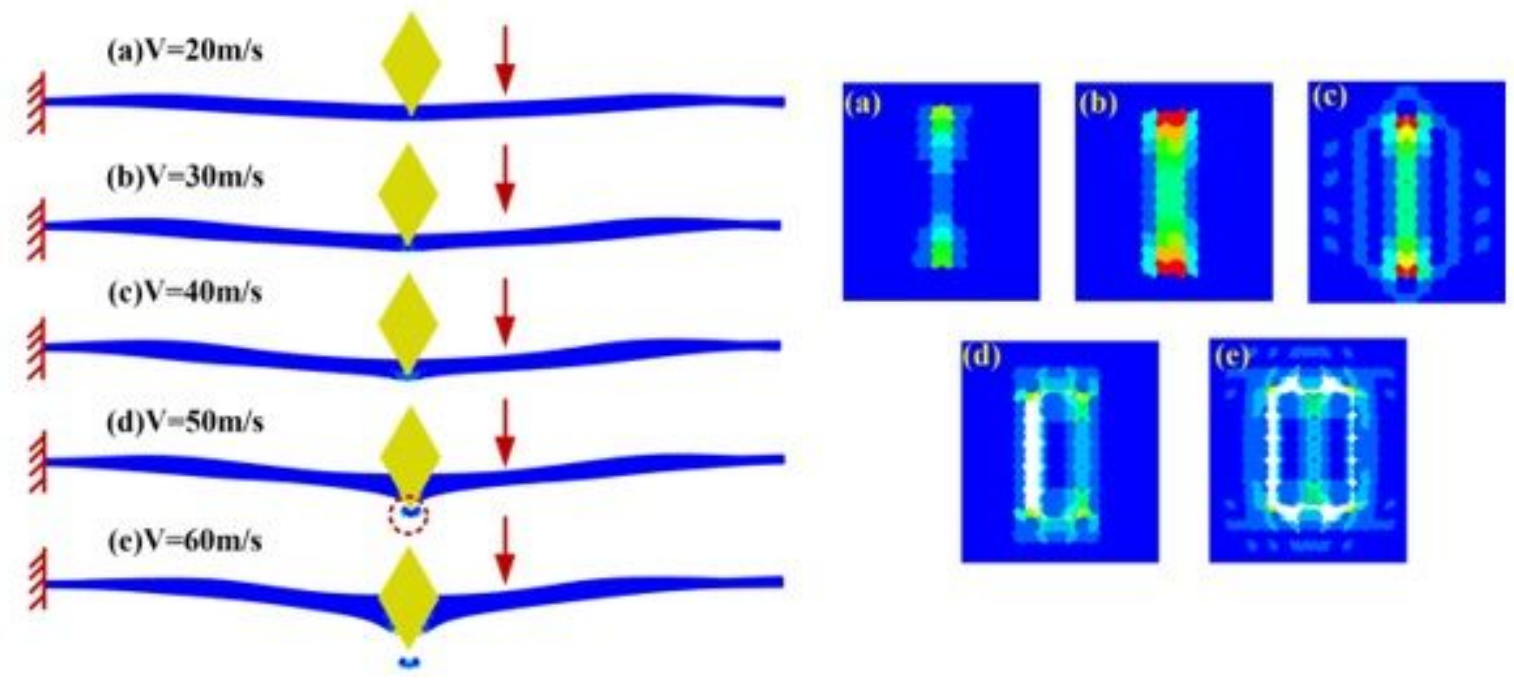

\section{Figure 19}

Deformation behavior and crater profiles of cantilever beam at different incident velocities $(l=60 \mathrm{~mm}$, $\mathrm{w}=30 \mathrm{~mm}, \mathrm{~h}=0.5 \mathrm{~mm}$ )
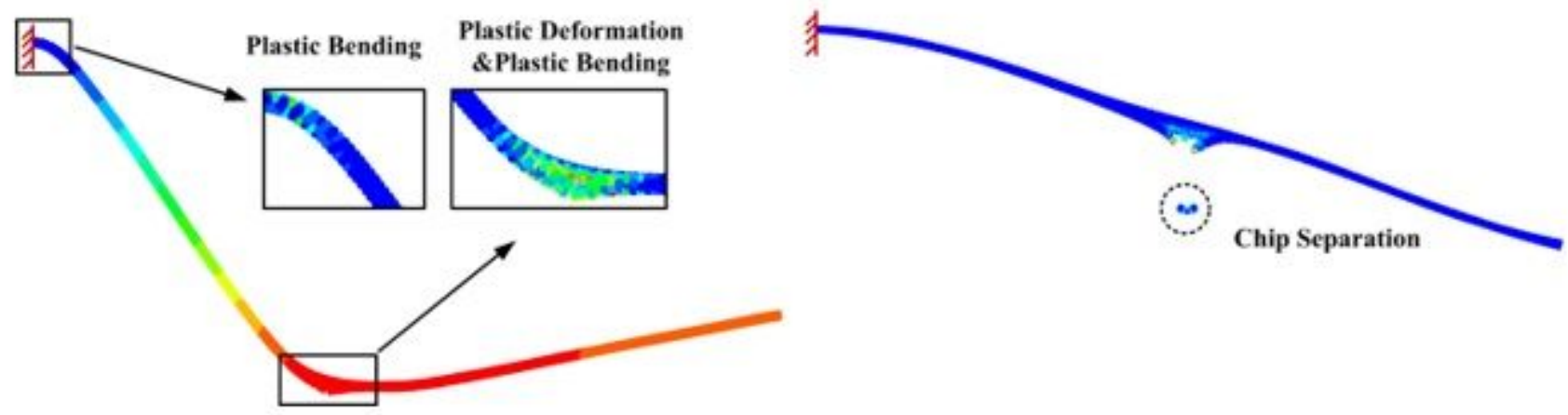

(a) The width of cantilever beam: $w=10 \mathrm{~mm}$

(b) The width of cantilever beam: $w=30 \mathrm{~mm}$ 
Figure 20

Deformation behavior of cantilever beams with different widths under the same impact condition $\left(V=60 \mathrm{~m} / \mathrm{s}, \mathrm{ai}=90^{\circ}, \theta \mathrm{i}=0^{\circ}\right)$

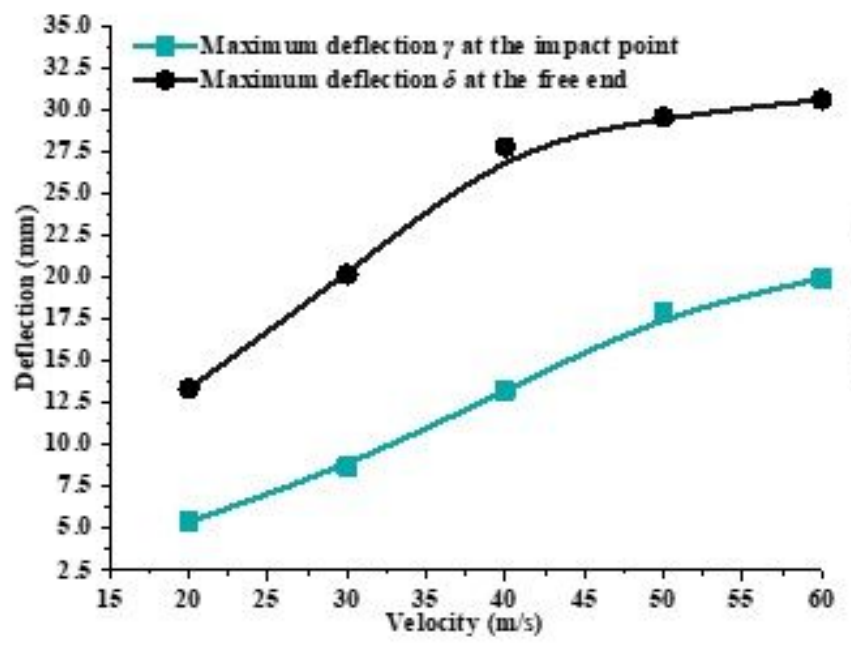

(a) The size of cantilever beam: $L=60 \mathrm{~mm}, \mathrm{w}=10 \mathrm{~mm}, \mathrm{~h}=0.5 \mathrm{~mm}$

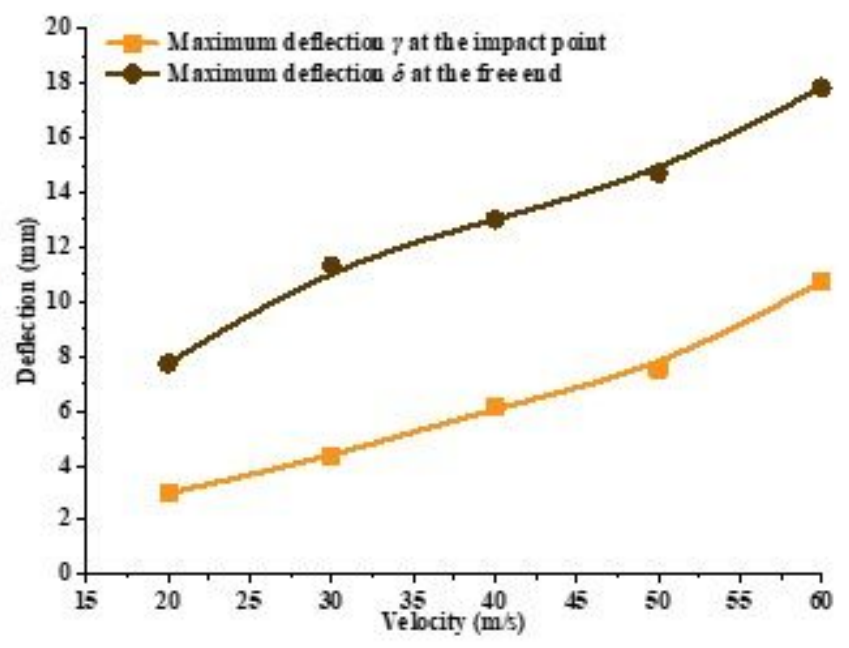

(b) The size of cantilever beam: $L=60 \mathrm{~mm}, \mathrm{w}=30 \mathrm{~mm}, \mathrm{~h}=0.5 \mathrm{~mm}$

\section{Figure 21}

Variation of deflection of cantilever beams of different sizes with the incident velocity
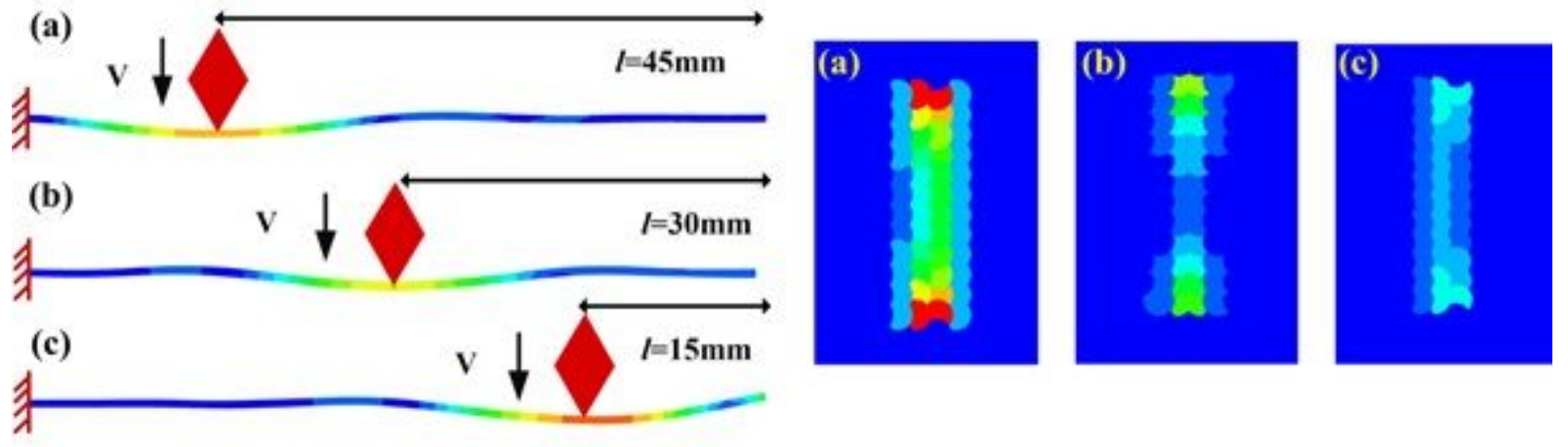

Figure 22

Different impact positions of cantilever beams and corresponding crater contours 


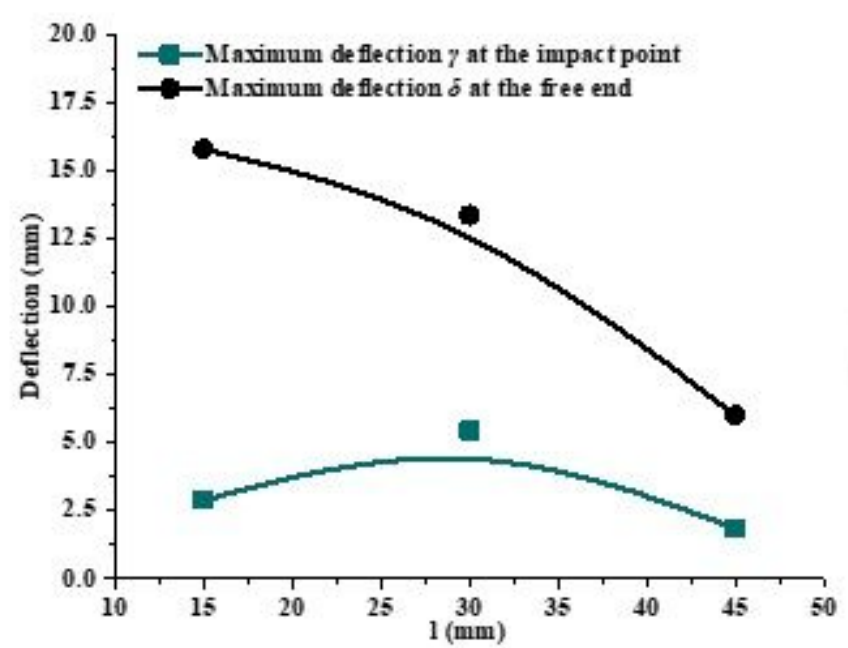

(a) The size of cantilever beam: $L=60 \mathrm{~mm}, \mathrm{w}=10 \mathrm{~mm}, \mathrm{~h}=0.5 \mathrm{~mm}$

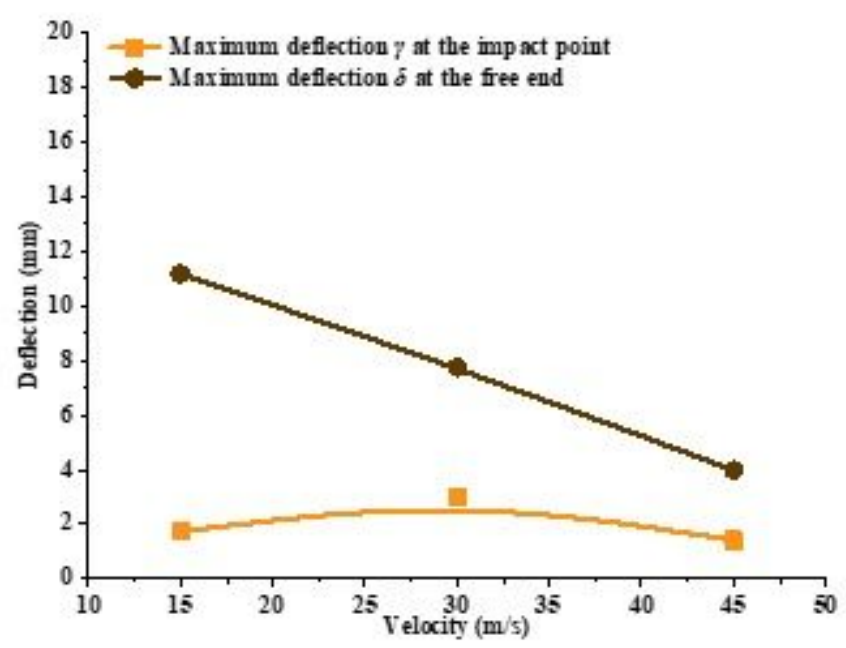

(b) The size of cantilever beam: $L=60 \mathrm{~mm}, \mathrm{w}=30 \mathrm{~mm}, \mathrm{~h}=0.5 \mathrm{~mm}$

\section{Figure 23}

Deflection of cantilever beams of different sizes varies with the incident position of particles

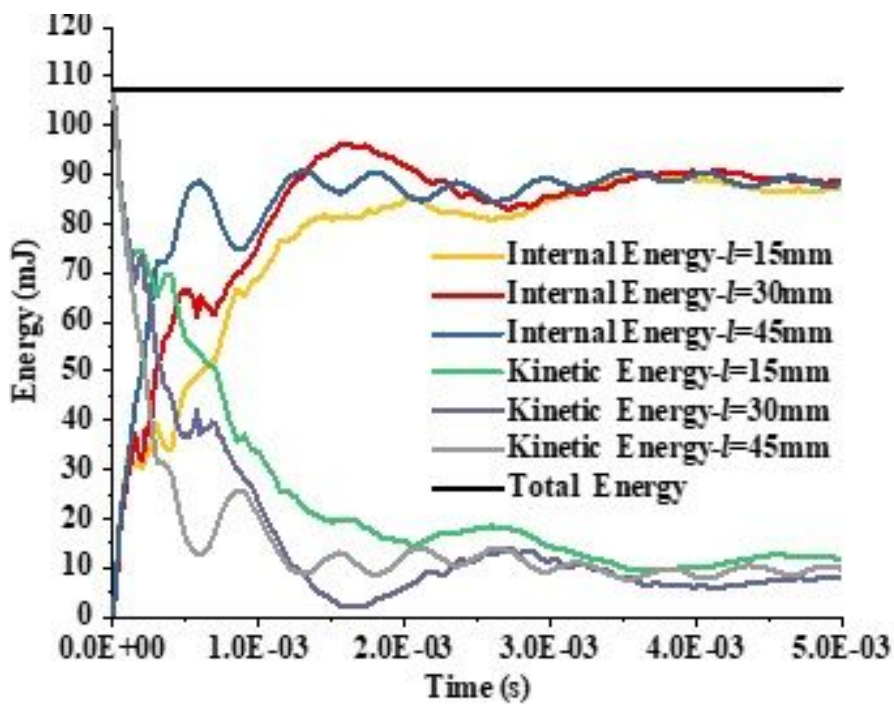

Figure 24

Energy evolution curves of cantilever beam system at different impact positions 


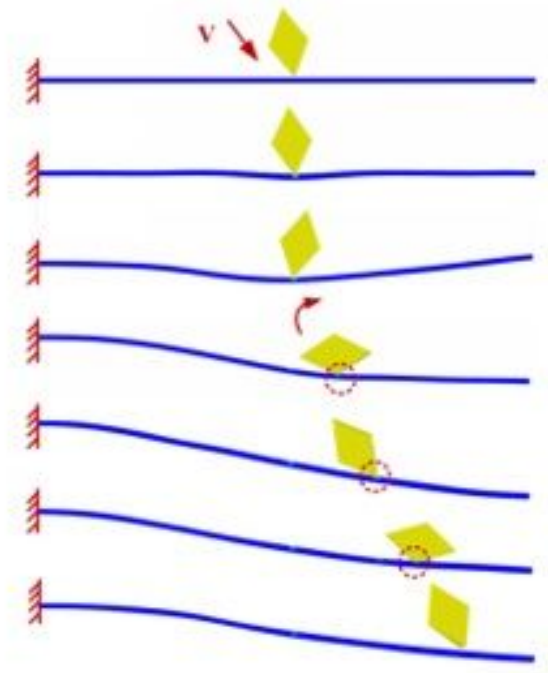

(a) case1: $V=20 \mathrm{~m} / \mathrm{s}, \alpha_{l}=60^{\circ}, \theta_{i}=10^{\circ}$

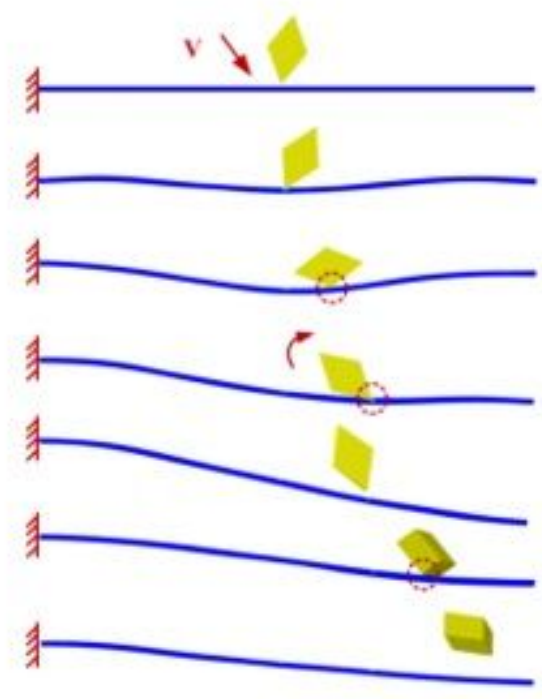

(c) case $3: V=20 \mathrm{~m} / \mathrm{s}, \alpha_{4}=60^{\circ}, \theta_{i}=-10^{\circ}$

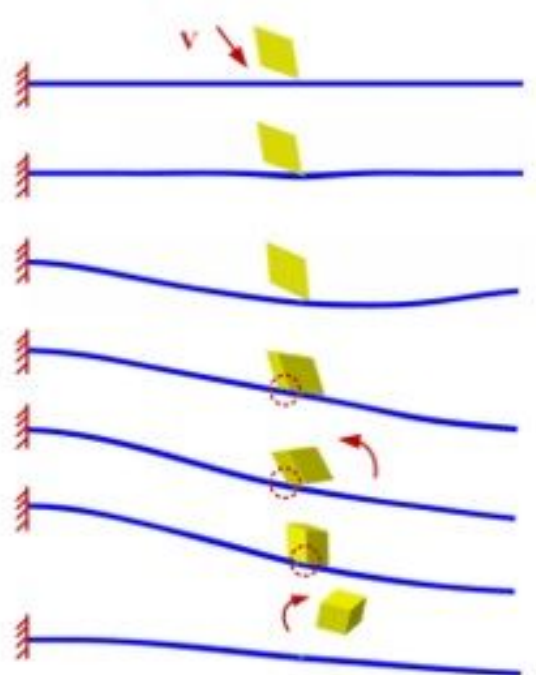

(b) case $2: V=20 \mathrm{~m} / \mathrm{s}, \alpha_{2}=60^{\circ}, \theta_{t}=40^{\circ}$

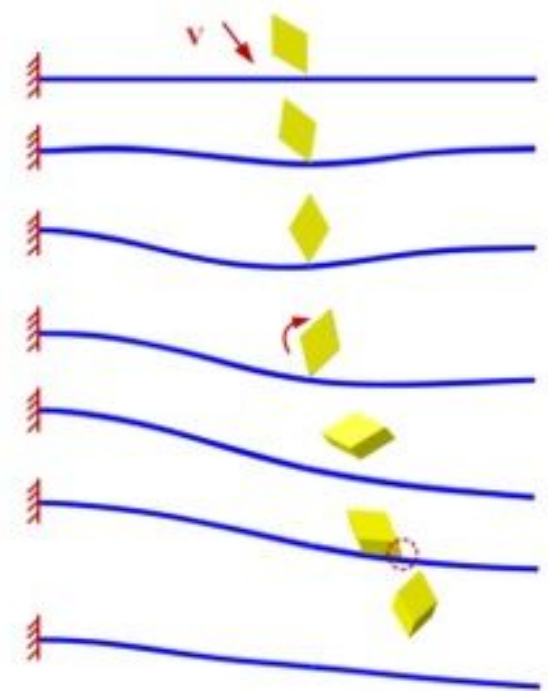

(d) case4: $V=20 \mathrm{~m} / \mathrm{s}, \alpha_{1}=60^{\circ}, \theta_{i}=30^{\circ}$

\section{Figure 25}

Influence of impact angle ai and orientation angle $\theta \mathrm{i}$ on particle motion behavior when impact cantilever beam 

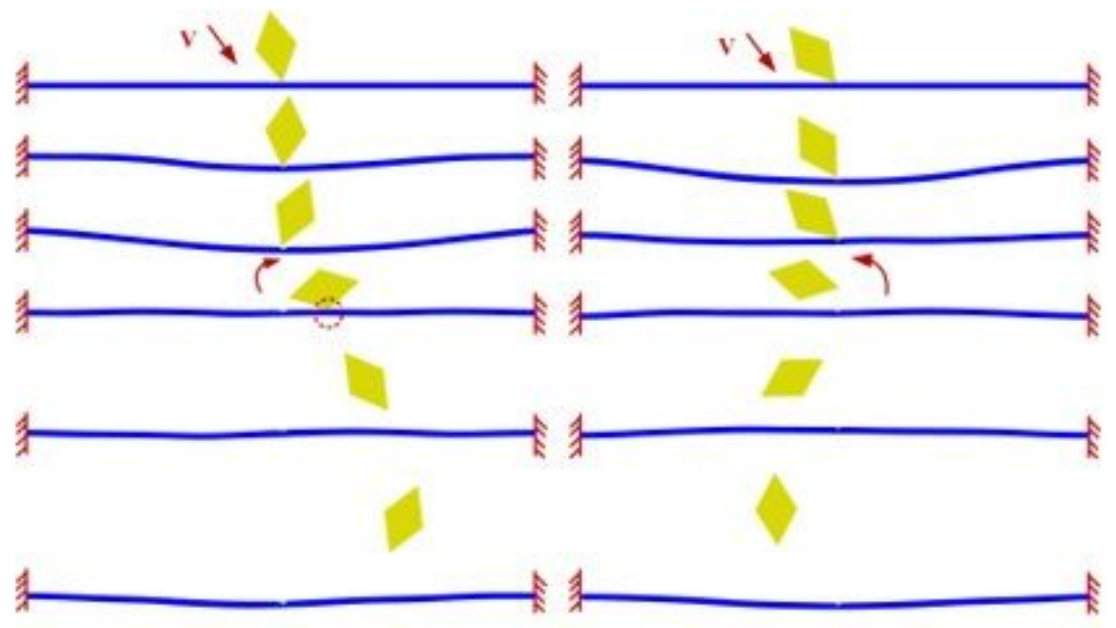

(a) case1: $V=20 \mathrm{~m} / \mathrm{s} \quad \alpha_{4}=60^{\circ} \theta_{i}=10^{\circ}$

(b) case 2: $V=20 \mathrm{~m} / \mathrm{s} \quad \alpha_{1}=60^{\circ} \quad \theta_{i}=40^{\circ}$
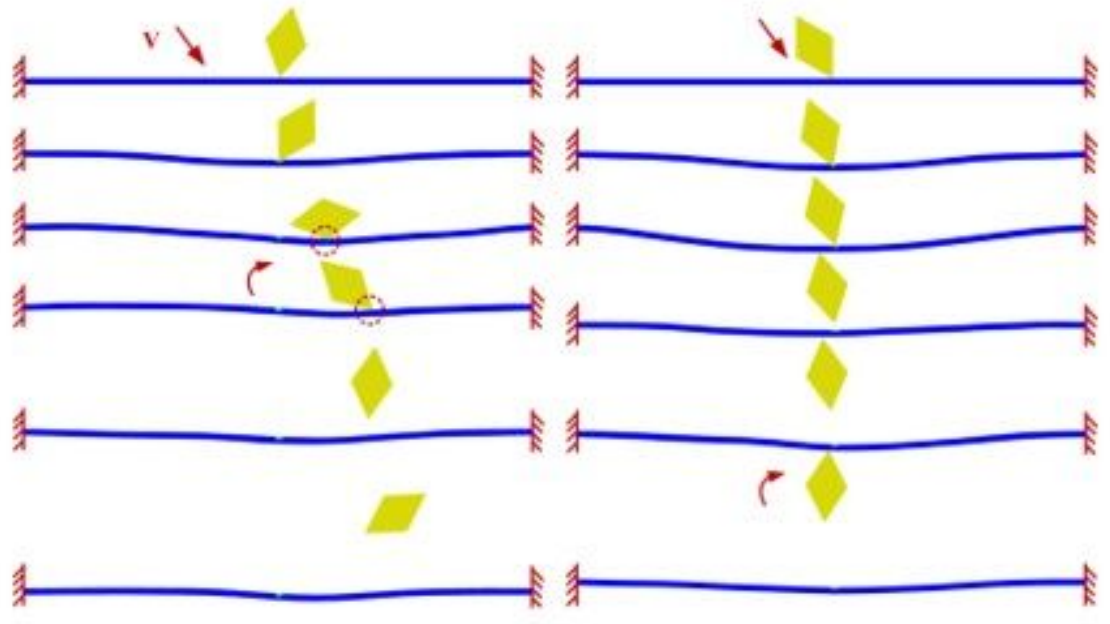

(c) case $3: V=20 \mathrm{~m} / \mathrm{s}, \alpha_{4}=60^{\circ}, \theta_{2}=-10^{\circ}$

(d) case $4: V=20 \mathrm{~m} / \mathrm{s}, a_{4}=60^{\circ}, \theta_{i}=30^{\circ}$

Figure 26

Influence of impact angle ai and orientation angle $\theta \mathrm{i}$ on particle motion behavior when impact fixed-fixed beam 


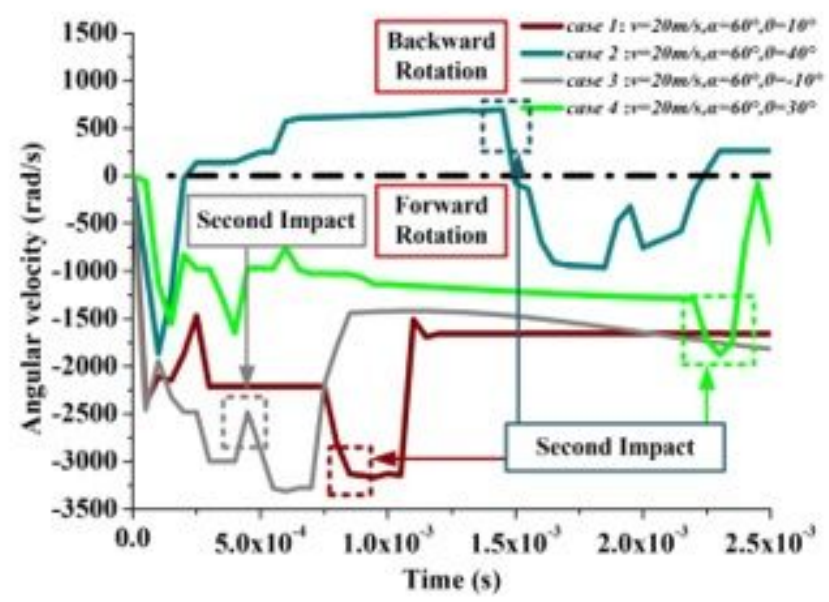

(a) Rhomboid-shaped particle impact cantilever beam

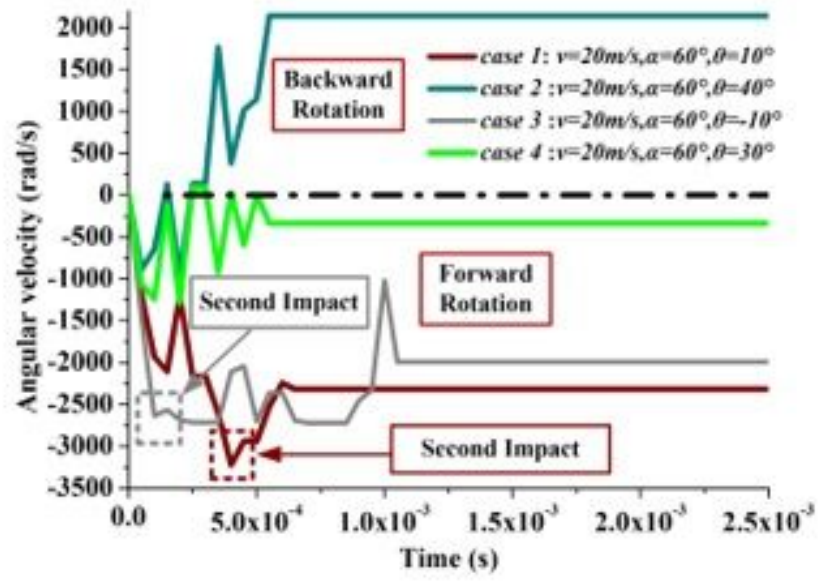

(b) Rhomboid-shaped particle impact fixed-fixed beam

\section{Figure 27}

History curves of particle angular velocity with time under four impact conditions
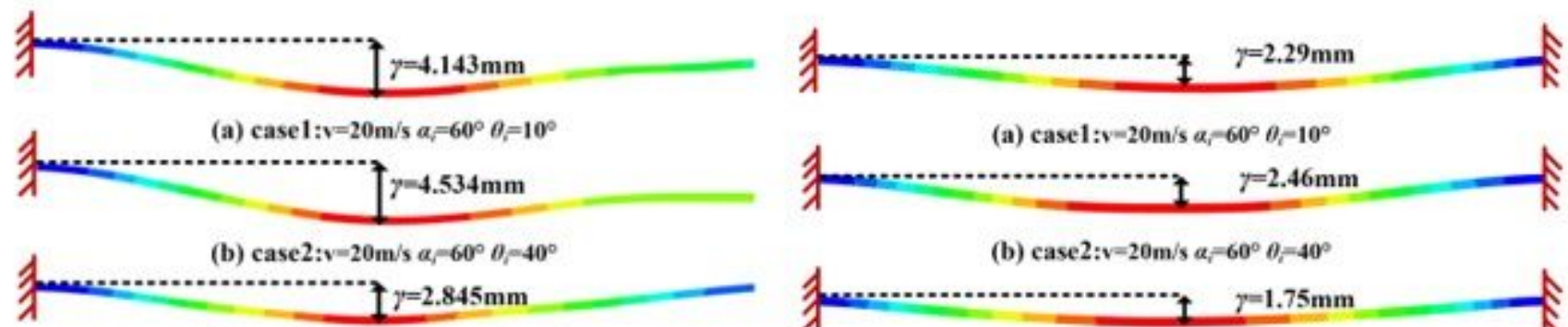

(c) case $3: \mathrm{v}=20 \mathrm{~m} / \mathrm{s} \alpha_{i}-60^{\circ} \theta_{f}=10^{\circ}$

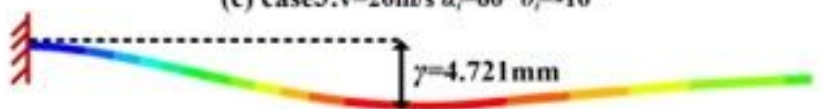

(d) case $4: \mathrm{v}=20 \mathrm{~m} / \mathrm{s} \alpha_{i}=60^{\circ} \quad \theta_{;}=30^{\circ}$

(a) Rhomboid-shaped particle impact cantilever beam

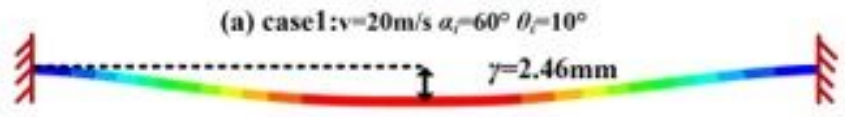

(b) case $2: \mathrm{v}=20 \mathrm{~m} / \mathrm{s} \alpha_{i}=60^{\circ} \theta_{i}=40^{\circ}$

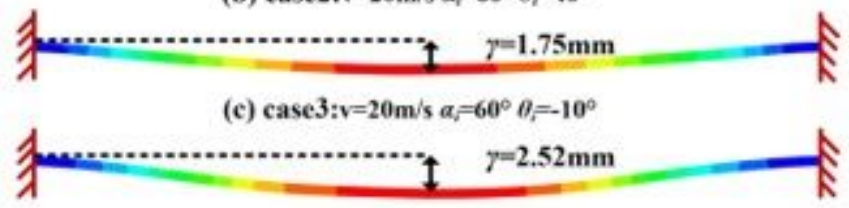

(d) case $4: \mathrm{v}-20 \mathrm{~m} / \mathrm{s} \alpha_{r}=60^{\circ} \theta_{i}=30^{\circ}$

(b) Rhomboid-shaped particle impact fixed-fixed beam

\section{Figure 28}

Maximum deflection $y$ at the impact point caused by rhomboid-shaped particle under four impact conditions 


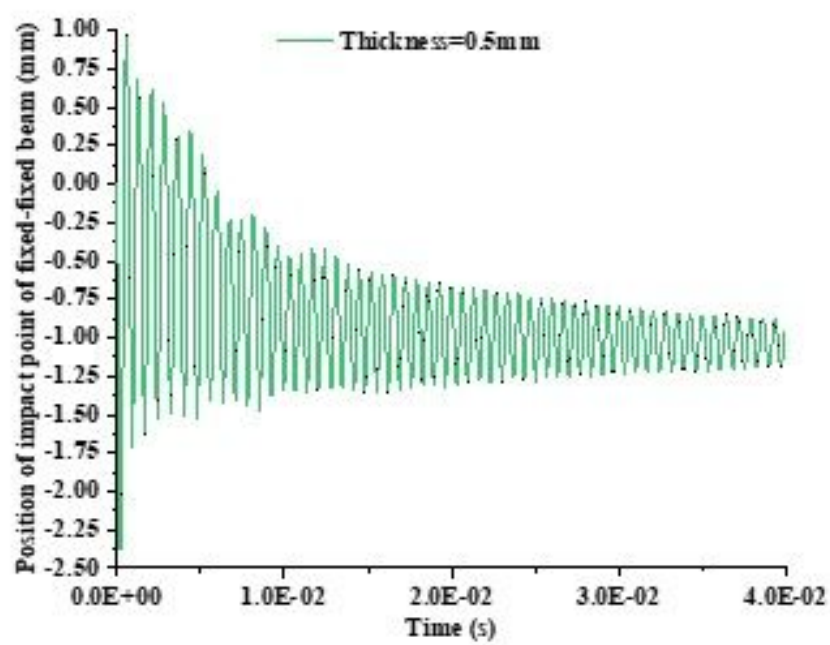

(a) Fixed-fixed beam: thickness $\mathrm{h}=0.5 \mathrm{~mm}$

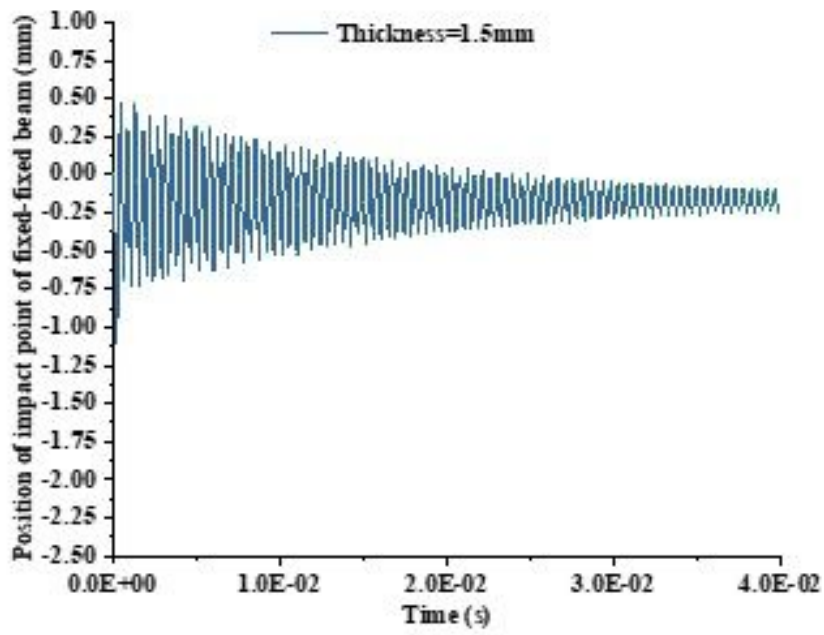

(c) Fixed-fixed beam: thickness $\mathrm{h}=1.5 \mathrm{~mm}$

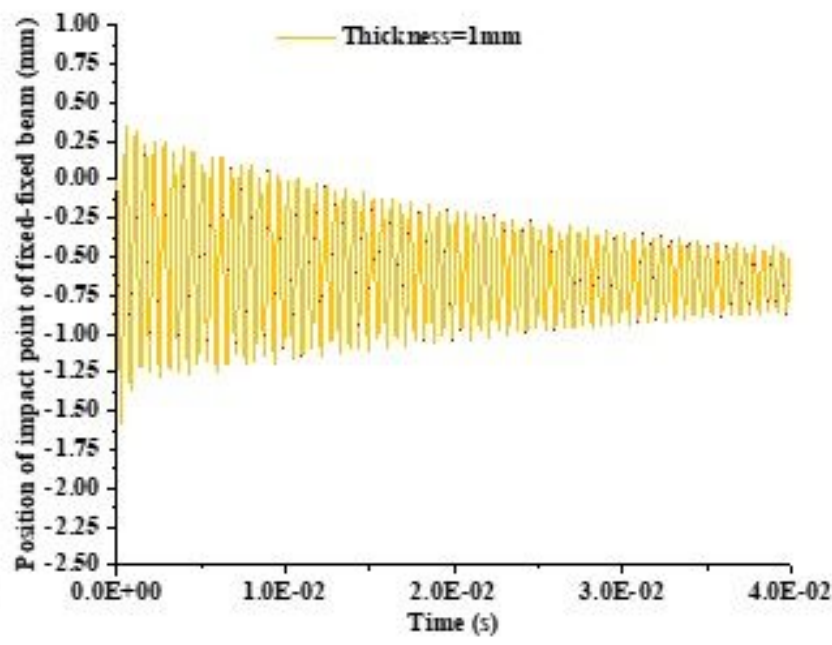

(b) Fixed-fixed beam: thickness $\mathrm{h}=1 \mathrm{~mm}$

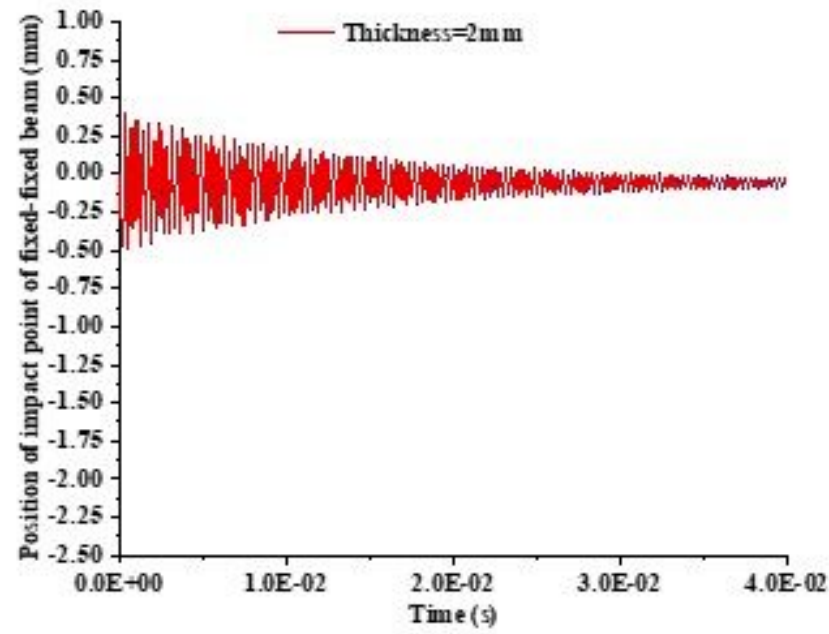

(d) Fixed-fixed beam: thickness $\mathrm{h}=2 \mathrm{~mm}$

Figure 29

Variation of amplitude with time of fixed-fixed beams with different thickness after impact 